\title{
Laboratory Directed Research and Development Program FY 2007
}

March 2008 


\section{DISCLAIMER}

This document was prepared as an account of work sponsored by the United States Government. While this document is believed to contain correct information, neither the United States Government nor any agency thereof, nor The Regents of the University of California, nor any of their employees, makes any warranty, express or implied, or assumes any legal responsibility for the accuracy, completeness, or usefulness of any information, apparatus, product, or process disclosed, or represents that its use would not infringe privately owned rights. Reference herein to any specific commercial product, process, or service by its trade name, trademark, manufacturer, or otherwise, does not necessarily constitute or imply its endorsement, recommendation, or favoring by the United States Government or any agency thereof, or The Regents of the University of California. The views and opinions of authors expressed herein do not necessarily state or reflect those of the United States Government or any agency thereof or The Regents of the University of California.

Lawrence Berkeley Laboratory is an equal opportunity employer. 


\author{
Report on \\ Ernest Orlando Lawrence \\ Berkeley National Laboratory
}

\title{
Laboratory Directed Research and Development Program
}

FY 2007

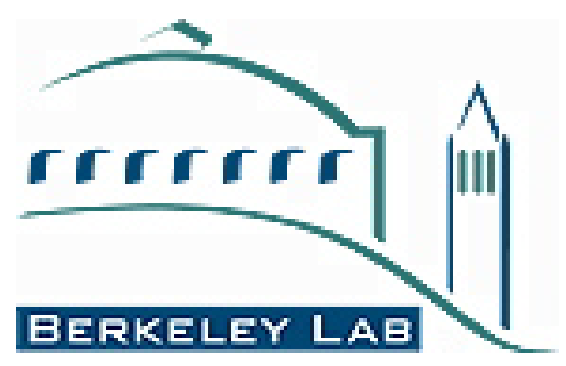

Ernest Orlando Lawrence Berkeley National Laboratory Berkeley, CA 94720

MARCH, 2008
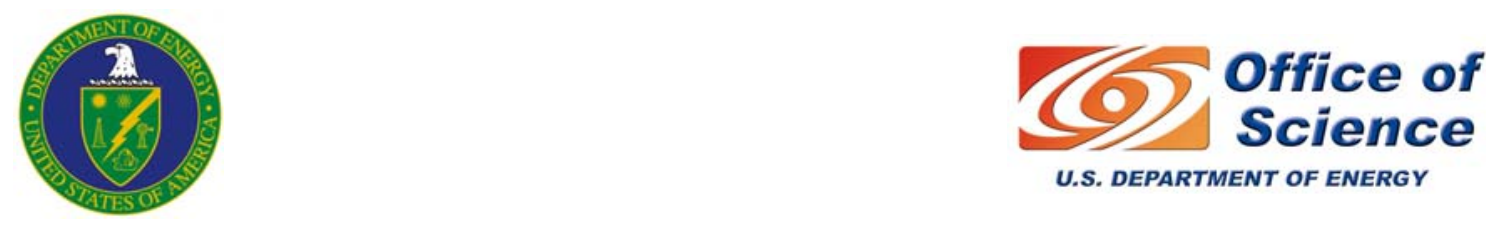

Prepared for the U.S. Department of Energy under Contract No. DE-AC02-05CH11231 



\section{Table of Contents}

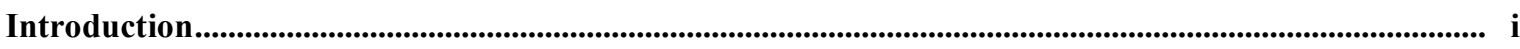

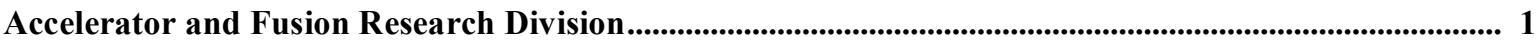

William Fawley

Alexander Zholents

Gregory Penn

John Corlett

Qing Ji

Grant Logan

Jonathan Wurtele

Wim Leemans

Carl Schroeder

William Fawley

Eric Esarey

Wim Leemans

Weishi Wan

Howard Padmore

Alexander Zholents

et al

Max Zolotorev

et al
FEL Concepts for Multiple Independent X-ray Beamlines .....

1

Ultra-compact Field Desorption Neutron Source for Cancer

Research

Enabling High Energy Density Physics at LBNL

2

3

Laser-plasma Accelerator Driven Free-Electron Laser with

High-Harmonic Seeding

4

High Brightness Photocathodes as Electron Sources for FELs ......

5

Emittance Manipulation and Beam Conditioning for FELs........... 6

Low Energy Spread Electron Source

7

Advanced Light Source Division

Elke Arenholz

Soren Prestemon

Jinghua Guo

Matthew Marcus

et al

Michael Martin

Zhao Hao

J. Alexander Liddle

Bruce Harteneck

Eric Anderson

Eli Rotenberg

Aaron Bostwick

Valeriy Yashchuk
Development of Fast Switching Superconducting Magnets for Spectroscopy Applications

Building In-Situ Electronic Structure Study Capability with Photon-in/ Photon-out Soft X-ray Spectroscopy

Measurement of Molecular Shape and Assembly Using X-ray

Scattering.....

Left-Handed Nanoscale Meta-Materials: Towards the Optical

Domain

NanoARPES: A New Detector for Nanometer-scale Electronic Structure Measurements 12

Ultra-high Resolution Optics for Soft X-ray Inelastic Scattering... 13

Chemical Sciences Division.

John Arnold

Synthetic and Electrochemical Approaches to Metal-Metal

Bonds in Actinides. 
Robert Bergman

Jonathan Ellman

Hendrik Bluhm

Kevin Wilson

John Prausnitz

David Shuh

Wayne Lukens

Andrew Canning

T. Don Tilley

Tolek Tyliszczak

David Shuh

Computing Sciences

(National Energy Research Scientific Computing Center, and Computational Research Divisions) .. 21

John Bell

Karlo Berket

Chin Guok

Olivier Chevassut

Abdelilah Essiari

David Konerding

Victor Markowitz

Natalia Ivanova

Nikos Kyrpides

Phil Hugenholtz

Leonid Oliker

John Shalf

KatherineYelick

William Kramer

Ali Pinar

Bernard Lesieutre

Juan Meza

Raquel Romano

Chris Ding

John Shalf

David Skinner

Jon Wilkening
Conversion of Glycerol and Aromatic Compounds from

Biomass to Major 3- and 6- Carbon Industrial Organic

Compounds

Chemical Reactions at Liquid/Vapor Interfaces Probed by

Photoemission Spectroscopy.....

Properties of New Ionic Liquids for Electrochemical

Applications and for Extraction of Heavy-Metal Cations from

Wastewaters

New Directions in Radionuclide Materials Characterization

Using the ALS....

New Approach for the Catalytic Conversion of Methane and

Other Inert Hydrocarbons....

Versatile Mini-Scanning Transmission X-ray Microscope

(mSTXM)

Structured, Adaptive Mesh Refinement Method for Multiphase

Reactive Transport in Groundwater ............................................... 21

On-Demand Overlays for Scientific Applications ......................... 22

Cryptographic Foundations for New Generation Distributed

Systems

Software Application Infrastructure for Efficiently Managing

Large-Scale Computational Biology Experiments

Integrated Microbial Community Genomes Data Management

System

Enhancing Commodity Scalar Processors with Vector

Components for Increased Scientific Productivity

Advanced Computational Tools for Electric Power Systems

Statistical Feature Modeling for Scientific Data Via Basis

Decomposition

Power Efficiency Metrics for High Performance Computing

Integrated Performance Monitoring of Grid and HPC

Workloads

Extended First Order System Least Squares Finite Elements 
Gary Andersen

James Berryman

John Coates

Donald DePaolo John Christensen

Benjamin Gilbert

Hoi-Ying Holman

Ping $\mathrm{Hu}$

Sharon Borglin

George Moridis

Matthew Reagan

Seiji Nakagawa

Andrea Cortis

Curtis Oldenburg

William Riley

Norman Miller

Carl Steefel

Micro-array Technology for Fungal Identification: Its Utility from the Environment to Homeland Security, Biomedicine, and Beyond.

Applications of Adjoint Field Methods and Time-Reversal Data Processing to Inverse Problems in Electromagnetics, Seismics, and Ultrasonics.

Investigation of the Microbial Processes Involved in a Biological Fuel Cell.

Micro-characterization and Chemical Micro-dynamics of Atmospheric Mineral Dust.

Behavior and Impact of Nanoparticles in the Environment

Compositional and Functional Analysis of Cell-Wall During Metal-Bacterial Interactions.

Interrelation of Global Warming and Hydrate Dissociation in Oceanic Accumulations.

New Technology for Permeability Enhancement for Natural Gas Extraction in Tight Reservoirs

Coupled Modeling of Hydrology, Nutrient Cycling, and

Vegatation: Applications to Water Quality and Water Balance.....

Biogeochemical Reaction Rates and Pathways in Porous Media... 41

Engineering Division

Peter Denes

Novel Imaging Detectors for Materials and Biology

Howard Padmore

Environmental Energy Technologies Division

Hugo Destaillats

Ashok Gadgil

Robert Kostecki

Venkat Srinivasan

Thomas Kirchstetter

Robert Kostecki

Sanuel Mao

Mark Levine

James McMahon

et al

Anastasios Melis

Rolf Mehlhorn

John Newman

Douglas Clark

Thomas Richardson André Anders
Understanding the Chemistry of Innovative Air Cleaning

Technologies

Arsenic ElectroChemistry: Exploring Electrochemical Arsenic

Remediation of Drinking Water for Bangladesh

Soot in Ice: Does Soot Enhance the Melting of Ice?

Surface Plasmon-Enhanced Photovaotaic Device 46

China's Energy Future: Changes in Energy Intensity

Integrated Decision Support Tool for Joint Optimal Control of Energy and Water Systems under Uncertainty ....

Bio-oil Accumulation in Unicellular Green Algae: A Pilot

Project. 49

Electrolysis of $\mathrm{CO} 2$ to a Liquid Fuel. 50

Switchable Mirror Prototype Fabrication 51 


\author{
Jonathan Eisen
}

Philip Hugenholtz

Len Pennacchio

Paul Spellman

Jan-Fang Cheng
Computational and Experimental Testing of Methods for Binning Sequences from Metagenomic Studies 52 Microbiota for Biomass Conversion and Cleaner Energy.

Development of Cost Effective Sequence-Based Technologies to Identify Genomic Alterations in Cancer

Life Sciences Division

Gareth Butland

Functional Interactomics: Integrating Physical and Functional Interaction Networks

Priscilla Cooper

Transcription CoFactor PC4 Interactions with RNA Polymerase and XPG in Transcription-Coupled Repair.

David Schild

Determining if PIR5 1 is a Potential Tumor Suppressor Gene Similar to $B R C A 2$

Andrew Wyrobek

Expression Profiling of Radiation and Cancer Susceptibility Francesco Marchetti Genes

Materials Sciences Division.

Matthew Francis

Philip Geissler

James De Yoreo

Ali Javey

Robert Kaindl

Yingzhong Ma

Daniel Chemla

Robert Ritchie

Joel Ager

Antoni Tomsia

Irfan Siddiqi

Gabor Somorjai

Dan Stamper-Kurn

Peidong Yang

T. Don Tilley

Christopher Chang
Tailoring the Self Assembly of Functionalized Biomolecular Building Blocks

Integration of Synthetic Nanomaterials for High Speed, Robust, and Flexible Circuitry

Terahertz-Frequency Conductivity and Ultrafast Optical

Excitations in Single-Walled Carbon Nanotubes

Aging, Disease and the Mechanical Response of Biological Tissues, Specifically in Human Bone.

Quantifying the Quantum Backaction of a Non-Linear

Dispersive Measurement .....

Electron Flow Generated by Gas Phase Exothermic Catalytic

Reactions Using Metal-Semiconductor Nanodiodes.

Studies of Quantum Antiferromagnetism in Two-dimensional Triangular Lattices Using Ultracold Atoms

Hierarchically Nanostructures Systems for Solar Energy

Hydrogen Production.

Nuclear Science Division

Roderick Clark

Kevin Lesko

Joseph Wang

Larry Phair
New Experimental Initiative to Deduce $(\mathrm{n}, \mathrm{f})$ Cross Sections for Advanced Fuel Studies

Physics Detector and Sensor Technologies Applied to Geological and Geophysical Applications at DUSEL 68 Improved Spectroscopy of Weakly Bound States in Nuclei. 
Ernst Sichtermann

Paul Vetter

Daniela Leitner

Matthaeus Leitner

Damon Todd
Hyperons in Polarized Proton Collisions and the Origin of the Nucleon Spin

Development of a Low-Energy, High-Current Astrophysics

Accelerator

Physical Biosciences Division

Paul Adams

Manfred Auer

Christopher Somerville

Jamie Cate

Michael Marletta

Jay Keasling

Gavin Crooks

Heinz Frei

et al

David Schaffer

Mina Bissell

Adam Arkin
Research Tools for the Conversion of Cellulose to Ethanol:

Structural Studies of Cellulose Synthesis

Cellulosomes and a Metabolic Pathway for Hydrocarbon

Production

Statistical Dynamics of Protein Evolution

Light Regulation and Visible Light-Driven Water Oxidation in Engineered Photosystems

Cooperation of Biochemical and Mechanical Signals in

Regulating Cell Fate Decision During Tissue Morphogenesis .......

Physics Division

Marco Battaglia

Advanced Monolithic Silicon Pixel Sensors

Christian Bauer

Soft-collinear Effective Theories for Collider Physics. 78

Hitoshi Murayama

New Directions for Theoretical Physics at the TeV-Scale.....

David Schlegel

Baryon Oscillations and Dark Energy: Prototyping Instruments ... 80

et al

Helmuth Spieler

Tests of a Multilayer Hybrid Neutron Detector Concept for

Nuclear Monitoring....

Cross-Divisional

Seung-Wuk Lee

Fabrication of Photovoltaic Devices Using Nanostructured

Biomaterials

William Lester

Development and Application of Quantum Monte Carlo (QMC)

Graham Fleming

Methods to Biological Systems.

Publications List 



\section{Introduction}

The Ernest Orlando Lawrence Berkeley National Laboratory (Berkeley Lab or LBNL) is a multi-program national research facility operated by the University of California for the Department of Energy (DOE). As an integral element of DOE's National Laboratory System, Berkeley Lab supports DOE's missions in fundamental science, energy resources, and environmental quality. Berkeley Lab programs advance four distinct goals for DOE and the nation:

- To perform leading multidisciplinary research in the computing sciences, physical sciences, energy sciences, biosciences, and general sciences in a manner that ensures employee and public safety and protection of the environment.

- To develop and operate unique national experimental facilities for qualified investigators.

- To educate and train future generations of scientists and engineers to promote national science and education goals.

- To transfer knowledge and technological innovations and to foster productive relationships among Berkeley Lab's research programs, universities, and industry in order to promote national economic competitiveness.

Berkeley Lab's research and the Laboratory Directed Research and Development (LDRD) program support DOE's Strategic Themes that are codified in DOE's 2006 Strategic Plan (DOE/CF-0010), with a primary focus on Scientific Discovery and Innovation. For that strategic theme, the Fiscal Year (FY) 2007 LDRD projects support each one of the three goals through multiple strategies described in the plan. In addition, LDRD efforts support the four goals of Energy Security, the two goals of Environmental Responsibility, and Nuclear Security (unclassified fundamental research that supports stockpile safety and nonproliferation programs). The LDRD program supports Office of Science strategic plans, including the 20year Scientific Facilities Plan and the Office of Science Strategic Plan. The research also supports the strategic directions periodically under consideration and review by the Office of Science Program Offices, such as LDRD projects germane to new research facility concepts and new fundamental science directions.

Berkeley Lab LDRD program also play an important role in leveraging DOE capabilities for national needs. The fundamental scientific research and development conducted in the program advances the skills and technologies of importance to our Work For Others (WFO) sponsors. Among many directions, these include a broad range of health-related science and technology of interest to the National Institutes of Health, breast cancer and accelerator research supported by the Department of Defense, detector technologies that should be useful to the Department of Homeland Security, and particle detection that will be valuable to the Environmental Protection Agency.

The Berkeley Lab Laboratory Directed Research and Development Program FY2007 report is compiled from annual reports submitted by principal investigators following the close of the fiscal year. This report describes the supported projects and summarizes their accomplishments. It constitutes a part of the LDRD program planning and documentation process that includes an annual planning cycle, project selection, implementation, and review.

The Berkeley Lab LDRD program is a critical tool for directing the Laboratory's forefront scientific research capabilities toward vital, excellent, and emerging scientific challenges. The program provides the resources for Berkeley Lab scientists to make rapid and significant contributions to critical national science and technology problems. The LDRD program also advances Berkeley Lab's core competencies, foundations, and scientific capability, and permits exploration of exciting new opportunities. All projects are work in forefront areas of science and technology. Areas eligible for support include the following:

- Advanced study of hypotheses, concepts, or innovative approaches to scientific or technical problems;

- Experiments and analyses directed toward "proof of principle" or early determination of the utility of new scientific ideas, technical concepts, or devices; and

- Conception and preliminary technical analyses of experimental facilities or devices.

The LDRD program supports Berkeley Lab's mission in many ways. First, because LDRD funds can be allocated within a relatively short time frame, Berkeley Lab researchers can support the mission of the Department of Energy (DOE) and serve the needs of the nation by quickly responding to forefront scientific problems. Second, LDRD enables Berkeley Lab to attract and retain highly qualified scientists and to support their efforts to carry out worldleading research. In addition, the LDRD program also supports new projects that involve graduate students and postdoctoral fellows, thus contributing to the education mission of Berkeley Lab.

Berkeley Lab has a formal process for allocating funds for the LDRD program. The process relies on individual 
scientific investigators and the scientific leadership of Berkeley Lab to identify opportunities that will contribute to scientific and institutional goals. The process is also designed to maintain compliance with DOE Orders, in particular DOE Order 413.2A, dated January 8, 2001, and its successor 413.2B, dated April 19, 2006. From year to year, the distribution of funds among the scientific program areas changes. This flexibility optimizes Berkeley Lab's ability to respond to opportunities.

Berkeley Lab LDRD policy and program decisions are the responsibility of the Laboratory Director. The Director has assigned general programmatic oversight responsibility to the Deputy Director. Administration and reporting on the LDRD program is supported by the Directorate's Office for Planning and Development. LDRD accounting procedures and financial management are consistent with the Laboratory's accounting principles and stipulations under the contract between the University of California and the Department of Energy, with accounting maintained through the Laboratory's Chief Financial Officer.

In FY2007, Berkeley Lab was authorized by DOE to establish a funding ceiling for the LDRD program of $\$ 22.0$ $M$ including General \& Administrative (G\&A) overhead, which equated to $4.2 \%$ of Berkeley Lab's FY2007 projected operating and capital equipment budgets. This funding level was provided to develop new scientific ideas and opportunities and allow the Berkeley Lab Director an opportunity to initiate new directions. Budget constraints limited available resources, however, so a little more than $\$ 16.1 \mathrm{M}$ was expended for operating and $\$ 0.1 \mathrm{M}$ for capital equipment (3.22\% of actual Berkeley Lab FY2007 operating and equipment costs).

In FY2007, scientists submitted 165 proposals, requesting about $\$ 29.3 \mathrm{M}$ in total funding. Eighty-three projects were funded, with awards ranging from $\$ 20 \mathrm{~K}$ to $\$ 534 \mathrm{~K}$. These projects are identified in the Table of Contents. 
FEL Concepts for Multiple Independent X-ray Beamlines

Principle Investigators: William Fawley, Alexander Zholents, Gregory Penn, John Corlett

\section{Project Description}

We propose to address fundamental issues and develop concepts for a next generation, FEL-based light source facility LBNL. We will design concepts for flexible photon beam performance, with multiple independent beamlines, each with performance tailored to individual experimental needs for average and peak flux and brightness, pulse repetition rate, resolving power, and wavelength, serving a wide variety of user needs. Each FEL will be independently tunable, and as am ensemble will provide a high degree of flexibility in providing for both short pulse, time-domain and narrow bandwidth, high resolution experiments.

Implementation of an optimized electron beam in a variety of FEL configurations will be studied, and a range of performance characteristics developed to meet the wide variety of user needs. Both SASE and seeded configurations will be simulated and developed, using existing accelerator and FEL expertise and simulation codes. Start-to end modeling of the electron beam is critical in determining performance capabilities, and these studies will be performed for a variety of potential electron sources and beam conditions.

\section{Accomplishments}

Our work in this LDRD concentrated on surveying needed FEL parameters for obtaining reasonable output at $1-\mathrm{nm}$ wavelength ( $1.2 \mathrm{keV}$ photon energy), investigating various optimizations in beam parameters using a 6D brightness formalism, and doing detailed simulation studies of the use of "reverse taper" undulator configurations for short pulse production. The parameter surveys utilized the M. Xie empirical formalism for FEL gain and resulted in a clear conclusion that very short period undulators (e.g., 15-mm period) would be necessary for reasonable undulator lengths if the electron beam energy remained at $2 \mathrm{GeV}$ or less. Output powers at the GW-level are possible for currents in the $0.5-1.0 \mathrm{kA}$ range; higher currents reduce the gain length but are more difficult to achieve with low emittances. Our studies also indicated that at the shortest wavelengths a large premium is attached to obtaining extremely low emittances, e.g., $0.5 \mathrm{~mm}$-mrad. These results are highly influenced by the short wavelength goal of $1 \mathrm{~nm}$; backing off to $2 \mathrm{~nm}$ would allow an emittance twice as large.

A 3D analysis of FEL radiation generation in the small signal regime examined scaling with 6D beam brightness (an invariant under Liouvillian flow). Brightness-scaled equations allow for the succinct characterization of the optimal FEL performance. We evaluated gain enhancement schemes based on beam phase space manipulations such as emittance exchange and conditioning, comparing their performance for both round and flat beams.

The reverse-taper work extended an idea by Saldin et al. (DESY) who found for hard x-ray FEL parameters that the combination of e-beam energy modulation by a few cycle optical laser together with an undulator strength taper that increased with $z$ could produce sub-fs duration output radiation pulses. We did detailed simulations of this scheme at XUV wavelengths (2 to 32 $\mathrm{nm}$ ) for both SASE and external laser-seeded FEL configurations. Our results found that the scheme works well, producing output pulses of 1-2 fs FWHM at 1-GW power levels for undulator lengths of $20 \mathrm{~m}$ or less. The output pulses have a wavelength chirp that could be utilized by users and/or allow further temporal pulse compression. Reversing the sign of the undulator taper together with a $\pi$ phase shift in the optical modulation pulse reverses the chirp. The scheme works well for both seeded and SASE configurations. 


\section{Ultra-compact Field Desorption Neutron Source for Cancer Research \\ Principal Investigator(s): Qing Ji}

Project Description

The objective of this proposed project is to develop and construct a proof-principle ultracompact, field-desorption neutron generator for cancer research, using a field desorption technique. The generator can be small enough to be housed in a catheter, which allows insertion of the neutron source into the human body and, by adding MRI capability, can be placed at the exact location of the tumors.

The device can be preloaded with pure deuterium, tritium gas, or a mixture, depending on the desired neutron energy and spectrum. For the case of the $\mathrm{D}-\mathrm{D}$ reaction, deuterium ions, $\mathrm{D}^{+}$, are generated by electrostatic field desorption of deuterium adsorbed on coated tip surfaces. They are then accelerated to $\sim 80 \mathrm{keV}$ when reaching the target surface where the nuclear reactions take place to produce neutrons. By powering on and off, the neutrons can be produced on-demand, thus eliminating expensive shielding for the protection of clinical personnel and patients when radioactive neutron sources are involved. The neutron yield from this type of system is proportional to the number of tips one can pack into the source. With a tip density of $10^{4} / \mathrm{mm}^{2}$, the estimated D-D neutron yield is approximately $10^{5} \mathrm{n} / \mathrm{s}$. Manufacturing of these tips requires microfabrication.

\section{Accomplishments}

We have successfully developed the process flow of microfabricating coated silicon-base field desorption tips. We use standard CMOS microfabrication technologies, start with 4"-diameter $<100>$ oriented n-type single crystal silicon wafers, go through a number of processes, such as plasma enhanced chemical vapor deposition, photolithography, metal sputtering deposition, chemical wet etch and plasma dry etch etc. Even though we are limited by the capability of photolithography tools we have access to in UC Berkeley Microlab, we've achieved the proposed tip density of approximately $1.6 \times 10^{4}$ tips $/ \mathrm{mm}^{2}$.

We've also designed and constructed a neutron tube for neutron yield measurement. Testing of the first generation of field emitter tips have yielded a measurable positive current, however, the exact ion current collected is unknown due to electron emission from the target and breakdown problems inside the test chamber. The experiment of neutron yield measurement is still in progress.

We've also upgraded our fabrication processes to accommodate 6" wafers, which will allow us to achieve ten times higher tip density by using DUV lithography system. The experiment of neutron generation is in progress. More work is underway to continue the fabrication of more field emitter arrays and test them under various pressures and voltages. 


\section{Enabling High Energy Density Physics at LBNL Principle Investigator(s): Grant Logan, Jonathan Wurtele, Wim Leemans Project Description}

High Energy Density Physics (HEDP) is an emerging field of science to understand the behavior of matter in extreme conditions of temperature and density. HEDP experiments offer us the potential to resolve fundamental questions about plasmas in several regimes important to our understanding of the universe. Our goal is to advance the technological basis of HEDP by developing computational tools and necessary diagnostic capabilities to conduct exciting high energy density plasma experiments using laboratory lasers and accelerators. Our proposal is to a) study new directions in HEDP experiments driven by intense focused particle beams using theory and advanced computation necessary for HEDP, and to b) develop cutting edge short pulse x-ray diagnostics for HEDP scientific exploration. This was accomplished by two tasks directed at computer codes and laser/X-ray scattering experiments over three years FY05-06-07:

Task 1: Conduct broad surveys of existing experimental techniques used to study equation of state and other properties of high energy density plasmas.

Task 2: Using the LOASIS laser facility and with particle, fluid and hybrid codes, study production of x-ray beams from laser-accelerated electron beams for HEDP target diagnostics. Accomplishments

Task 1 We developed algorithms for the purpose of predicting the behavior of targets heated to the warm dense matter (WDM) regime. In collaboration with the Computational Research Division, we have implemented a version of QEOS (an equation of state commonly used in inertial confinement fusion codes) in a modern automatic mesh refinement (AMR) fluid code for incorporation two-phase equation of state. The implementation of the two-phase model is currently one-dimensional. A simple one dimensional hydrodynamics code DISH has been developed as a tool for teaching students about computational hydrodynamics and as a research tool for testing new equations of state or ion deposition algorithms. It has been rigorously tested by an undergraduate during the summer of 2007, and is in continued use. We studied models to incorporate droplets and bubbles and other surface tension effects into hydrodynamics codes without resolving the actual droplets themselves, and an algorithm for studying the behavior of metallic foams. We initiated organizing an international Warm Dense Matter school, to be held at LBNL from January 10 - January 16, 2008.

Task 2 Optimization of x-rays from laser-accelerated electron beams undergoing betatron emission has been studied with a model using prescribed field simulations of laser wakefield acceleration to evaluate effects of density modulations versus changing of dephasing and other techniques to increase betatron radiation yield and peak energy. The model has been tested against simulations using a particle-in-cell based code (VORPAL), and indicates that betatron emission can be enhanced using longitudinally modulated plasmas but that the strongest signal gains are realized by increasing the electron beam energy. This work will be submitted for publication. Experiments on the $10 \mathrm{TW}$ laser system were carried out to further develop and characterize X-ray diagnostics including $\mathrm{CCD}$ /phosphor and silicon strip as well as single element detectors. These X-ray diagnostics are now ready for implemented on the GeV electron beam line at LOASIS, and supports FEL proof-of-principle experiments that are now pursued for the development of a compact EUV source. The efforts funded under this LDRD also support proposals anticipated to DNDO and/or NA-22. Simulations to study proton acceleration in collaboration with UNR indicate potential for ion acceleration at high but subcritical densities, with potential for higher energies than obtained from conventional solid targets. 
Laser-Plasma Accelerator Driven Free-Electron Laser with High-Harmonic Seeding

Principal Investigators: Carl Schroeder, William Fawley, Eric Esarey, Wim Leemans

\section{Project Description:}

The purpose of this project is to develop and design a novel, compact, high peak flux free-electron laser (FEL) driven by a laser-plasma accelerator. A theoretical and numerical study was performed to determine the technical feasibility of using a laser-plasma-accelerated electron bunch to drive an FEL to generate pulses of coherent, ultra-short, VUV radiation. In this study we considered the interaction of an ultrashort $(<50 \mathrm{fs})$ electron bunch produced by the LBNL laser wakefield accelerator (LWFA) in a conventional undulator (the THUNDER undulator, recently obtained by LBNL on extended loan from Boeing). The 0.5-GeV LWFA electron beam interacting with the THUNDER undulator produces 31-nm radiation at the fundamental. The ultra-high current of the LWFA electron beam $(\sim 10 \mathrm{kA})$ allows for saturation of the FEL after 5 meters of undulator operating in the self-amplified spontaneous emission (SASE) regime. Highharmonic generation (HHG) from a laser-gas interaction is considered as a coherent seed for the FEL. HHG seeding has several advantages over SASE: reduced undulator saturation length, improved temporal coherence properties, and reduced the power fluctuations. Since the HHG seed and LWFA electron beam can be driven by the same laser, intrinsic synchronization can be achieved between the seed and LWFA beam. At saturation the proposed FEL would be capable of producing $>10^{13}$ photons/pulse.

\section{Accomplishments:}

We have preformed a series of numerical studies, using the FEL code GINGER, to evaluate the FEL performance. Inasmuch as several of the LWFA beam parameters are not well determined (e.g., transverse emittance and instantaneous energy spread), we performed FEL output sensitivity studies. In particular, we considered variations in the beam energy spread, emittance, and mismatch in the focusing system. For the HHG-seeded cases, we found that the saturated power dropped by a factor of two when the relative instantaneous energy spread exceeded $0.5 \%$, whereas the emittance may be as large as $4 \mathrm{~mm}$-mrad (presuming a matched beam). The third harmonic power was found to be considerably more sensitive to the emittance than the energy spread. For SASE cases, increasing the relative instantaneous energy spread beyond $0.25 \%$ quickly prevents power saturation in the $5-\mathrm{m}$ length of the THUNDER undulator. Simultaneously varying the beam charge and duration shows large sensitivity to the charge but relatively little to the duration over a range of 5 to $30 \mathrm{fs}$. Shot-to-shot fluctuations in the SASE mode were also examined; in the absence of any accelerator jitter, the RMS shot-to-shot SASE output jitter is $\pm 25 \%$ in photon number at the fundamental and $\pm 50 \%$ at the third harmonic.

We also modeled vacuum-chamber wakefields and space-charge effects (using analytic models). Even for the ultra-high beam currents and small undulator gaps considered, we found resistive wall wakefields do not significantly degrade the performance of the FEL for peak currents $<30 \mathrm{kA}$. The induced energy chirp over a coherence length created by the longitudinal Coulomb self-fields was also found to be small compared to the FEL parameter for the parameters considered.

In addition, we examined the use of spontaneous undulator radiation as an electron beam diagnostic (for energy spread and emittance). The spontaneous radiation was calculated for various electron beam parameters using the code SPECTRA. It is envisioned that the initial set of experiments will characterize the LWFA beam via measurement of the undulator radiation. 


\section{High Brightness Photocathodes as Electron Sources for FELs \\ Principal Investigator(s): Weishi Wan, Howard Padmore}

\section{Project description}

Free Electron Lasers (FELs) offer instantaneous flux and brightness 10 orders of magnitude higher than a $3^{\text {rd }}$ generation synchrotron source like the ALS. They offer in addition pulse lengths of fsecs or less in comparison to the 100 psec of a conventional synchrotron. However, in order to operate, the electron beam has to occupy a very small phase space volume, and, in order to do this, presently operating and planned FELs start with a relatively hot beam from a laser driven photocathode gun, and compress its phase volume by acceleration to high energy. In addition, present day cathodes have limited quantum efficiency, and so, limited by available laser power, FELs typically have low repetition rates. We are addressing these issues by investigation of a new generation of photocathodes, that have engineered electronic properties. The aim is to develop a cathode that produces a beam with very low transverse energy spread; this will enable higher photon energies from a FEL, or simply reduce its scale. In addition, we aim to increase the quantum efficiency to the point at which conventional laser technology can provide enough pulse power to operate at $\mathrm{MHz}$ repetition rate. Successful development of photocathodes would result in reduced cost and technical risk, and enhanced opportunities, for new FEL technical approaches.

On the experimental side, we have used the technique of angle resolved photoemission spectroscopy (ARPES) to investigate copper photocathodes. On the one hand, we have done experiments at Brescia, Italy. On the other hand, we are building a more optimized version of the apparatus in our own lab. On the theoretical side, we have used a direct $\mathrm{N}$ body simulation code to study the stochastic Coulomb scattering among the electrons to predict the final emittance of the electron beam from the photocathode.

\section{Accomplishments}

We have investigated metallic photocathodes, as used at the LCLS. We have determined that photo-current at the very low photon energies typically used is dominated by surface states. The emittance deduced from the experimental data agrees well with the theoretical prediction. This directly has led to a prediction of the minimum transverse momentum, and to a direction for producing lower emittance though use of other crystalline surfaces. At the same time, we have ordered all major equipment for our lab and have started the assembling and commissioning of the experimental system. On the theoretical side, we have obtained much more detailed understanding of the effect of the stochastic Coulomb scattering on the electron beam. The overall emittance growth obtained from the simulation agrees qualitatively with the estimate based on a simplistic thermodynamics model. 
Emittance Manipulation and Beam Conditioning for FEL's Principle Investigators: John Corlett, Steve Lidia, Gregory Penn, Ji Qiang, David Robin, Alexander Zholents

\section{Project Description}

The purpose of the project is to design electron beam emittance control and manipulation techniques for application in a low-energy accelerator feeding several independent FEL beamlines, which could be built at LBNL. Implementation of anticipated techniques would help to obtain a high brightness electron beam in a cost effective facility where control of the electron beam quality will be gained in the electron gun and maintained through the whole accelerator.

Accomplishments

Our most significant accomplishment has been developing of understanding of the microbunching instability that has its roots in the shot noise of electrons. This inevitable and fundamental process limits the brightness of the electron beam. Reliable predictions for the effect of this instability were impossible until completion of our work. During our study we developed better theoretical understanding and unique computational tools that allowed us to follow the development of the instability with the needed precision. We performed a series of numerical simulations for various accelerator configurations and obtained a clear understanding about what works the best. The knowledge gained and tools developed will guide us in future work where we actually want to produce an optimized design of the accelerator feeding ten independent FELs. We reported results of our work at several workshops and already published them or submitted them for publication (see list of publications and presentations).

We performed an optimization study of a new type of the injector based on a radio frequency photocathode gun. The goal was to identify a set of injector components needed to support a low emittance production and to understand the ultimate brightness of the electron beam that can be produced in such injector. Independent optimizations were performed for the electron beam with the electron bunch charges varying from $10 \mathrm{pC}$ to $1 \mathrm{nC}$ and electron bunch repetition rate up to $1 \mathrm{MHz}$.

We began comparative analysis of various layouts for the entire machine including the injector, accelerator, electron beam switch yard to distribute beam between ten independent FELs, and ten arrays of FEL undulators. Initially we produced a skeleton design for the switch yard and identified specific performance requirements for the high repetition rate kicker magnet. We also produced a feasibility study for this kicker magnet and investigated various options to minimize wake fields due to resistive wall losses. 
Low Energy Spread Electron Source

Principal Investigators: Max Zolotorev, Eugene D. Commins, Jim Oneill, Fernando Sannibale, Anton Tremsin, Weishi Wan.

\section{Project Description for FY07.}

The purpose of the project is to explore a novel approach for a pulsed electron source that has the potential to approach the quantum-limited brightness and lowering the effective source temperature from the current $\sim 100 \mathrm{meV}$ to $\sim 10 \mu \mathrm{eV}$. Such a source can open a wide range of novel applications that utilize angstrom-scale spatial resolution and $\mu \mathrm{eV}$-scale energy resolution. Examples include angstrom-scale resolution electron microscopy, electron holography and investigations of dynamics on the ps time scale using pump-probe techniques. The technology available within the proposed budget will constrain the effective temperature to the meV-range. In this multi-year LDRD we have designed, fabricated and constructed a proof of principle source, and we are completing the characterization of its performance.

In the complete scheme, an effusive source generates a beam of neutral Cesium atoms. These atoms are then excited to a Rydberg level very close to ionization $\left(\sim 10^{-5} \mathrm{eV}\right)$ in the interaction region (IR) defined by the overlapping of 3 laser beams. Two of the lasers are CW while the third is pulsed with a rep-rate of $10 \mathrm{MHz}$. The parameters are tuned to produce an average of 1 Rydberg excited atom per pulse. After the laser pulse, a delay ( $\sim 40 \mathrm{~ns})$ is introduced to allow the electron in the excited atom to travel far from the ion $(\sim 70 \mu \mathrm{m})$ reaching the turning point of its orbit so as to lose practically all of its kinetic energy. At this point, a pulsed voltage is applied to finally ionize the atom and to deliver the electron to the optical system for the final acceleration and for the energy analysis. Last, a second pulsed electric field removes the remaining ion from the IR. In this way the Coulomb interaction effects are minimized and a very high brightness electron beam is obtained.

In the proof of principle scheme the Rydberg atoms are generated and subsequently ionized by the electric pulse, and the energy of resulting electrons is measured by a time of flight system based on micro-channel plate detectors.

\section{Accomplishments}

During this third year of LDRD, all the parts have been fabricated or purchased, and the assembling of the proof of principle source has been completed.

A diagnostics system, based on micro-channel plate (MCP) detectors has been fully integrated with the source and successfully tested with a thermionic electron for calibration purposes. Time of flight measurements of the generated electrons will allow for the source tuning and for the characterization of its performance.

A novel scheme for the frequency control feedback for the two continuous-wave lasers (852 and $1470 \mathrm{~nm}$ wavelengths) have been developed and successfully operated.

The theory of the source has been completed in the detail and a paper on it has been published on Physical Review Letters.

A seminar at the Lawrence Berkeley National Laboratory on the source has been given on October 2007.

A patent on the source concept has been filed with the competent governmental agency. 


\section{Advanced Light Source Division}

Development of Fast Switching Superconducting Magnets for Spectroscopy Applications Principal Investigator(s): Elke Arenholz, Soren O. Prestemon

\section{Project Description}

The goal of this project is to develop the core technology for superconducting magnets providing peak fields of 5T in arbitrary directions and capable of full field reversal in 5s for soft $\mathrm{x}$-ray spectroscopy applications. Magnetic fields of this magnitude are needed to align magnetic moments along hard magnetization directions in novel materials (molecular magnets, ferromagnetic semiconductors, functional oxides). Arbitrary field directions will allow us to take full advantage of the strengths of soft x-ray magnetic dichroism measurements. Rapid field ramping suitable for point-by-point field reversal in spectroscopy experiments will provide much improved sensitivity enabling new insights into the magnetic properties of nanostructures.

We expect to achieve peak fields of $5 \mathrm{~T}$ by using novel superconductors for the magnet coils and holmium for pole pieces. The steel return yoke will increase the field strength at the center of the device, eliminate stray fields in its vicinity, and provide structural support of the system. Arbitrary field orientations will be obtained by the superposition of the field generated by four coils pairs arranged octahedrally. The four dipole pairs will be powered by four individual power supplies allowing to truly point the field vector in any direction. Rapid field ramping can induce magnet quenches from heating associated with superconductor AC losses. They will be minimized by proper selection of conductor and optimization of the cryogenic design.

\section{Accomplishments}

The key technical difficulties in the magnet development arise from the combined requirements of high field, arbitrary field direction, and rapid field variation. We have designed a prototypical superconducting eight pole magnet and performed a detailed magnetic and cryogenic analysis to a) identify the most suitable conductor, b) minimize heat sources associated with rapid field ramping, and c) optimize the cryogenic design for device performance.

To generate $5 \mathrm{~T}$ at the center of eight octahedrally arranged coils with a $60 \mathrm{~mm}$ gap, the peak field on the conductor will be approx. 12T at current densities exceeding $1000 \mathrm{~A} / \mathrm{mm}^{2}$. A range of commercially available superconducting materials were reviewed. Specific $\mathrm{Nb}_{3}$ Sn wires were identified as the most suitable material, with a critical current density of $1190 \mathrm{~A} / \mathrm{mm}^{2}$ at $12 \mathrm{~T}$ and $4.2 \mathrm{~K}$. To identify and resolve fabrication issues posed by heat-treatment of the conductor after winding, a prototype single coil was fabricated and thoroughly tested.

Due to the importance of fast-ramp capabilities, much design effort focused on reducing heat sources associated with varying fields. AC-losses in the superconductor were minimized by appropriate selection of the conductor. Eddy currents in pole, yoke, coil mandrel, and cryostat were minimized through appropriate slits and laminations. Despite these efforts some eddycurrent heating has to be expected and was accounted for in the design of the cryogenic system. The significantly higher $\mathrm{T}_{\mathrm{C}}$ of $\mathrm{Nb}_{3} \mathrm{Sn}\left(\mathrm{T}_{\mathrm{C}}=18 \mathrm{~K}\right)$ provides a larger temperature margin to resist sudden heat deposition than conventional NbTi devices $\left(\mathrm{T}_{\mathrm{C}}=9.8 \mathrm{~K}\right)$. Moreover, the coils will be immersed in liquid He eliminating temperature gradients common in conduction-cooled systems. Helium vapor from the system will be admitted to a cryocooler and returned as liquid. This eliminates the cost and personnel time associated with liquid Helium refill systems and significantly improves the user friendliness of the system.

The new capabilities amount to an improvement in peak operating field by $50 \%$ and in switching rate by a factor of $\sim 1000$ compared to existing superconducting devices, and will enable new classes of experiments pushing forward our understanding of magnetic materials. 


\section{Building In-situ Electronic Structure Study Capability with Photon-in/Photon-out Soft-X-Ray Spectroscopy \\ Principle Investigator(s): Jinghua Guo}

\section{Project Description}

The purpose of the project is to build in-situ electronic structure study capability of the chemical reactions using photon-in/photon-out soft-x-ray spectroscopy on beamline 7.0 at ALS. The goal is to demonstrate a unique way to handle the real systems (gas-, liquid-phase samples, wet surfaces and interfaces) for electronic structure studies. The focus in science will be on understanding the binding mechanism of hydrogen adsorption and probing efficient hydrogenstorage materials.

The photon-in/photon-out soft-x-ray spectroscopy can be used to study different chemical species of interested in depth, or under the presence of electric and/or magnetic fields, or under wet condition and ambient pressure. Here, the in-situ electronic structure study using photonin/photon-out soft-x-ray spectroscopy will be realized by constructing a gas cell for the electronic structure study of hydrogen adsorption/desorption and a liquid cell for the electronic structure study of liquid mixing and diffusion.

The proposed effort requires the new experimental equipment to be built. The gaseous and liquid phase samples will be maintained in cells inside the vacuum chamber with windows to allow the penetration of soft x-rays. We will build the chemical cells for in-situ photonin/photon-out soft-x-ray spectroscopy studies, including to build the hydrogen cell for in-situ electronic structure studies of hydrogen adsorption/desorption process involving selected potential hydrogen storage materials and to build liquid cell and make it compatible with vacuum environment, and perform a test experiment of in-situ electronic structure studies of molecular liquids and solutions.

\section{Accomplishments}

Thin membrane windows of silicon nitride and carbon have been tested to stand a pressure up to 2 atms of gas. We have build the hydrogen cell for in-situ electronic structure studies of hydrogen adsorption/desorption process on the samples of highly oriented pyrolytic graphite (HOPG) and single-walled carbon nanotubes (SWNTs). A few on-going test experiments were performed to determine a good buffer layer materials (Pd, Nb, etc.) on windows for hydrogen adsorption in metal hydrides, such as $\mathrm{Mg}_{2} \mathrm{Ni}$.

It was shown in-situ interaction between SWNTs and gases at room temperature and gas adsorption caused changes in electronic structure of SWNTs that can be recovered by evacuation of gas. The collision can be responsible to the pressure-dependent spectral change. The raising gas pressure up to 10 torr induces deformation of SWNTs thus decreases the conductance, but further increase of pressure up to 450 torr will recover the deformation due to inner tube collision.

We have built a liquid flow cell for electronic structure study of liquids in a temperature range of $5-50{ }^{\circ} \mathrm{C}$, and a number of test experiments of in-situ electronic structure studies of molecular liquids and solutions are performed. 
Measurement of Molecular Shape and Assembly using X-ray Scattering

Principal Investigator(s): M. Marcus, H. Padmore (ALS), P. Adams (PBD), N. Balsara, R. Segalman (MSD, UCB), J. Berger, J. Kuriyan (PBD,UCB), J. Kornfield (Caltech),

E. Kramer (UCSB), W. Bras (ESRF)

\section{Project Description}

The goal of this work is to establish a capability at the ALS for the measurement of the shape and assembly of complex molecules and polymers. Applications range from determination of the structure of large protein complexes, to the study of the dynamics of polymer nanostructure assembly. In protein structure for example, it is often difficult or impossible to crystallize very large complexes. In the polymer nanostructure arena, many methods have been developed for synthesis of the basic building blocks, but engineering the chemistry of the self assembly of these units into useful structures requires knowledge of the assembly pathways. X-ray scattering allows one to measure these processes.

We also plan to establish an x-ray scattering capability at the ALS for use by the structural biology and nanoscience communities. Normally the cost of establishing such a capability would be around $\$ 2 \mathrm{M}$ for the beamline and detector, but due to the relocation of an existing research team to a superconducting bending magnet source, a normal bending magnet beamline and hutch will be available for use. In addition, two excellent detectors have being made available, due to upgrades that have occurred in the instrumentation on the ALS structural biology beamline.

\section{Accomplishments}

We have succeeded in establishing a scattering capability at the Advanced Light Source. In the fall of 06 we built and installed a multilayer monochromator operating at $10 \mathrm{keV}$ with a $\mathrm{E} / \Delta \mathrm{E}$ of 100 downstream of a pair of newly installed Be windows. Further downstream, a set of scattering slits and exit slits, which reduce scatter from the existing mirror, were installed. In January 07 we got first monochromatic light into the x-ray hutch. Shortly following this, we installed two ADSC Q4 CCD detectors, which we are borrowing from BCSB/PBD. These two detectors allow us to perform simultaneous small- and wide-angle $\mathrm{X}$-ray scattering. The small angle detector is positioned on a track system, allowing for easy and quick change of sample-detector distance. A vacuum path was placed between sample and detector to eliminate air scatter. The wide-angle detector is suspended from an aluminum gantry that was designed and successfully built to allow five dimensions of freedom for positioning the detector. Acquisition software was developed that controls both detectors as well as sample environments simultaneously, thus allowing measurements of varied structures from $150 \mathrm{~nm}$ down to $0.2 \mathrm{~nm}$. In June of 07 we commenced the commissioning phase of beamline development during which we have invited the PI's and collaborators of this LDRD to use the new capabilities of the beamline to collect data. The response has been extremely positive, resulting in a completely booked schedule for several months in advance. The design of the endstation has proven to be very flexible, allowing investigation of samples ranging from bones and shells to polymers and liquid crystals in phase transitions as well as shape determination of proteins and nanoparticles in solution.

The beamline is planned to transition from the commissioning phase to a proposal based schedule in June 08. 


\title{
Left-Handed Nanoscale Meta-Materials: Towards the Optical Domain
}

\author{
Investigators: Michael C. Martin, Zhao Hao, Alex Liddle, Bruce Harteneck and Erik Anderson
}

\section{Project Description}

The index of refraction of normal materials is positive, requiring curved surfaces or special structures to construct lenses which focus light. Recently, new artificial materials have been reported that have a negative index of refraction, which allows a homogeneous flat slab to behave as a "perfect", lens, possibly even creating sub-diffraction limited focusing. Numerous applications in science, technology, communications and medicine can be expected. Scaling these composite meta-materials to nano dimensions should increase the frequencies at which novel left-handed phenomena can be observed and exploited. The goal of this LDRD is to fabricate and measure nanoscale meta-materials, leading ultimately to left-handed optics for visible wavelengths.

\section{Accomplishments}

We received LDRD funding in FY2005 and FY2006 with the goal of creating fully lefthanded nano-scale metamaterials at infrared frequencies. We have developed detailed electric and magnetic response simulation capabilities, micro- and nano-fabrication techniques to place these test structures on thin $\mathrm{SiN}$ windows, and careful optical measurements of the resultant meta-materials using the ALS infrared beamlines 1.4.2, 1.4.3 and 1.4.4.

During FY 2007 we have fabricated a variety of nanoscale metamaterials. We succeeded in making structures with a negative index of refraction composed of individual closed ring magnetic dipole resonators. The experimentally observed resonance frequencies of 60 and $100 \mathrm{~nm}$ linewidth resonators are $\sim 7000 \mathrm{~cm}^{-1}(210$ $\mathrm{THz})$ and $\sim 5000 \quad \mathrm{~cm}-1 \quad(150 \mathrm{THz})$, respectively, well in the near-IR range. We show that negative index of refraction is obtained for the $100 \mathrm{~nm}$ resonators at $\sim 5000$ $\mathrm{cm}^{-1}(150 \mathrm{THz})$. This experiment on magnetic dipolar resonators confirms the existence of magnetic dipolar moments in
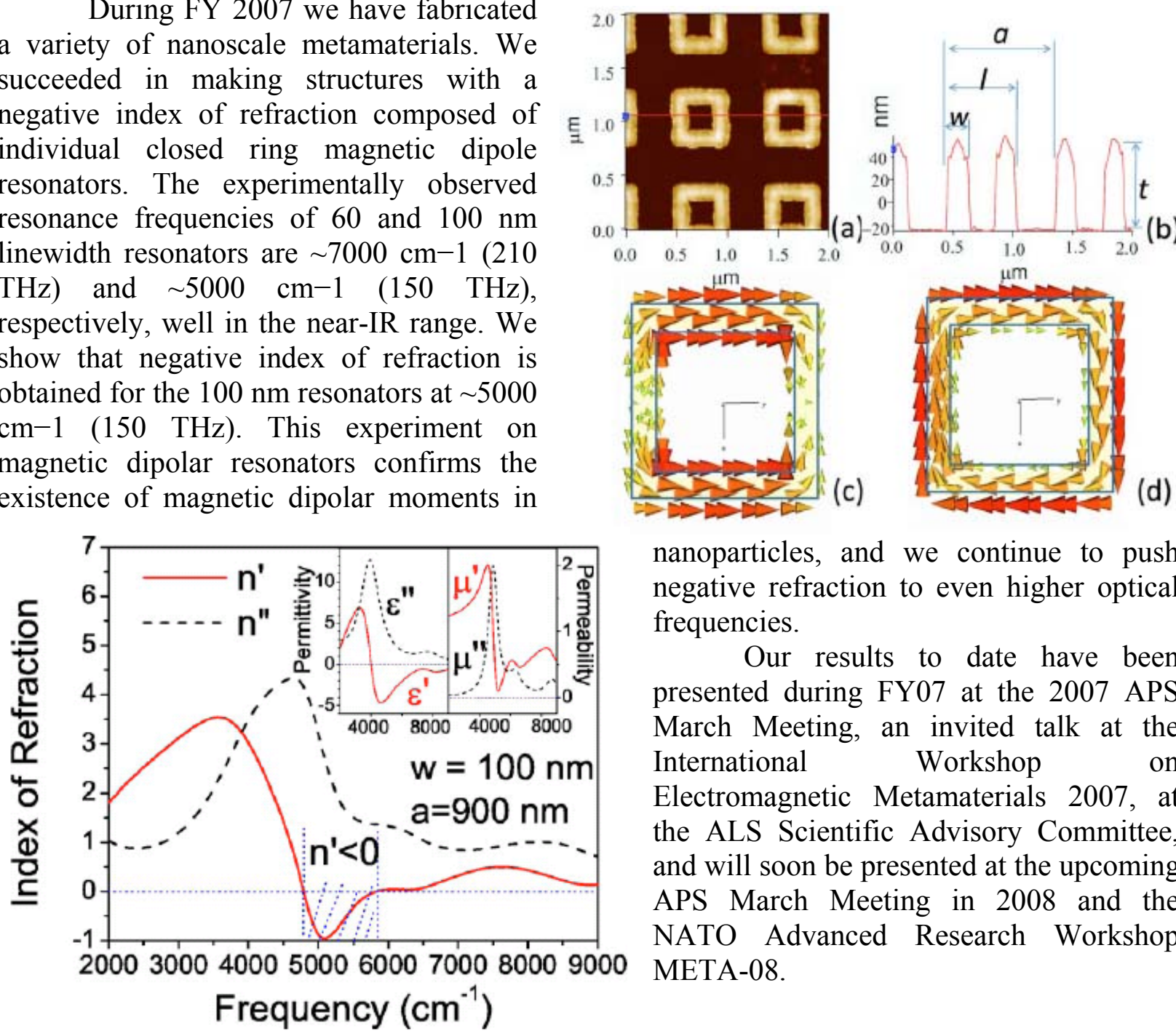

nanoparticles, and we continue to push negative refraction to even higher optical frequencies.

Our results to date have been presented during FY07 at the 2007 APS March Meeting, an invited talk at the International Workshop on Electromagnetic Metamaterials 2007, at the ALS Scientific Advisory Committee, and will soon be presented at the upcoming APS March Meeting in 2008 and the NATO Advanced Research Workshop META-08. 
nanoARPES: A New Detector for nm-scale Electronic Structure Measurements Principal Investigator: Eli Rotenberg and Aaon Bostwick (LBNL, ALS)

\section{Project description}

The goal of the nanoARPES is the development of a new probe for electronic structure measurements at the $50 \mathrm{~nm}$ or smaller length scale. The uniqueness of this probe arises from its ability to resolve the spectral function and the momenta of the electronic states, fundamental properties that cannot be determined by any other technique than Angle Resolved Photoemission Spectroscopy (ARPES). Determining these properties for nanometer devices is expected to have an impact on the fundamental understanding of correlated systems, quantum phase transitions, as well as practical problems in semiconductor physics, such as in solid-state lighting and solar power generation. But extension of conventional ARPES, in which a $\sim 50 \mathrm{~mm}$ focused beam of soft x-rays induces photoelectron emission into an angle- and energy-resolving detector, to the nanometer scale requires novel solutions to problems on both the photon focusing and electron detection sides of the problem. In addition, real-life problems require operation at low temperature.

\section{Project Accomplishments}

This is the third and final year of funding for this project. In the first year, we established $300 \mathrm{~nm}$ spatial resolution and with this performed pioneering experiments in sub- $\mu \mathrm{m}$ resolution for the first time. In year 2, we designed a new piezo-scanning system with the ability to operate at low temperature and at ultra-high vacuum for the first time (Mark II instrument).

In this third year, we fabricated the Mark II UHV instrument and tested its operation. We achieved UHV operation for the first time, and we implemented a rudimentary sample preparation facility. The present instrument now meets the basic capabilities for general use and
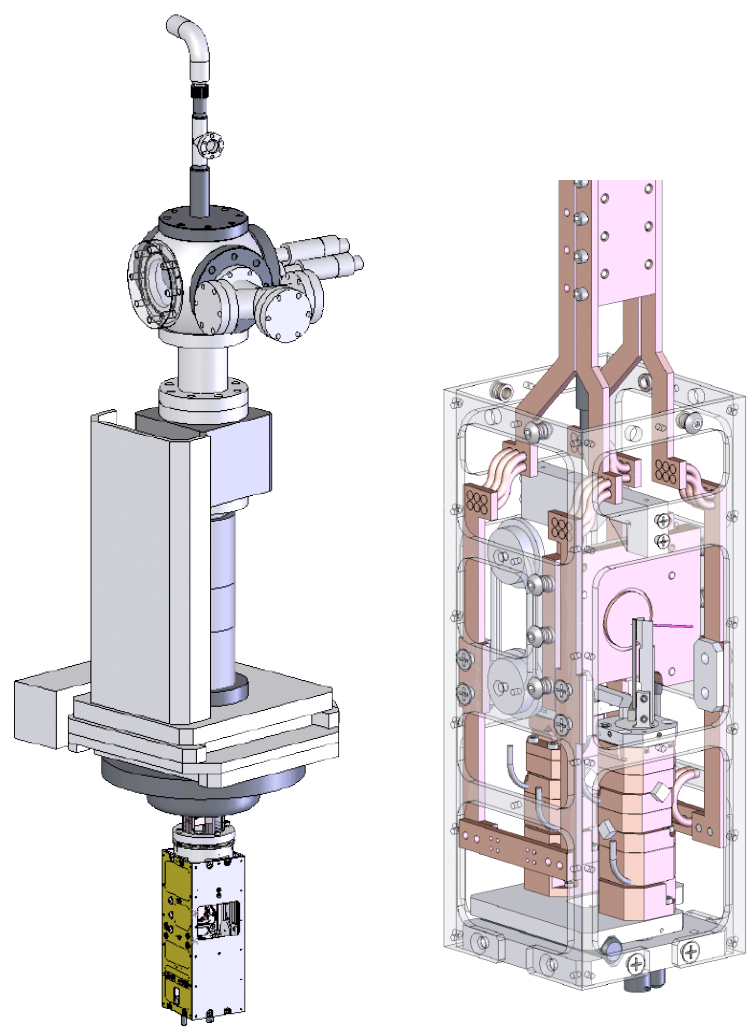
the first user operation is expected in early FY08. We also designed the next version (Mark III) of the instrument, which adds the capability for an optical telescope view of the sample, as well as independent motion of the zone plate optics and order-sorting aperture. Mark III parts were fabricated and qualified. This feature is required for continous scanning of the photon energy, which is needed for certain experiments such as near-edge xray absorption spectroscopy (NEXAFS).

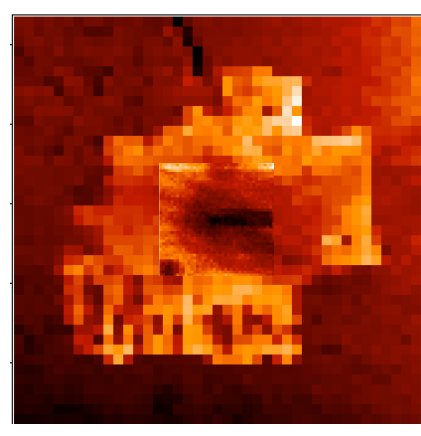

(right) First image taken with Mark II instrument. The sample is a single monolayer flake of graphene (center rectangle) surrounded by gold mask (left, center) The future Mark III version of the nanoARPES microscope in its shielded and a cutaway view. The present (operational) Mark II version looks similar. 


\section{Ultra-high resolution optics for soft x-ray inelastic scattering}

Principal Investigator(s): Valeriy V. Yashchuk

\section{Project Description}

The goal of this work is to demonstrate and establish the technology required for ultrahigh resolution Resonant Inelastic soft X-ray Scattering (RIXS). RIXS is one of the most important potential new probes in electronic structure determination due to its elemental selectivity, high momentum resolution, and ability to probe bulk materials. However, important advantages of the method are limited due to energy resolution limits imposed by traditional grating optics, especially at soft x-ray energies. For example, energy scales imposed by collective phenomena in correlated electronic systems dictate that meV energy resolution is required for experiments at 500-1000 eV energy, requiring a resolving power of about $10,{ }^{6}$ unachievable with conventional optics.

Here we develop a revolutionary new way to construct gratings to get to the extremely high resolving powers required. We investigate two major ways to achieve high resolution, a) use of a grating in high order, or b) use of extremely high line density gratings, formed by cutting a multilayer mirror at a small angle. For approach a), simulations have shown that it is possible to design a multilayer grating that effectively diffracts almost all the energy into a defined order, only if the land to period ratio is significantly reduced. In this respect, the approach b) looks more promising. Method b) assumes the need for a grating with line density of $\sim 50,000 \mathrm{l} / \mathrm{mm}$. We plan to achieve this in 3 steps: 1) creation of a perfect low resolution echellette grating, 2) deposition of a multilayer reflector on the echellette, and 3) slicing of the multilayer at a small angle by polishing. Each step has been independently developed by various authors, but we work to put all the steps together, and test it within a spectrograph.

\section{Accomplishments}

Our most significant accomplishment has been to develop numerical methods for soft x-ray diffraction simulation and evaluation the performance of high resolution diffraction gratings according to the suggested approaches, depending on fabrication tolerances on different steps of grating fabrication. The simulation has allowed us to distinguish the most favorable approach and concentrate efforts on the most promising way, method b). It has been demonstrated that an extremely high diffracted intensity of more than $25 \%$ and resolution of about $10^{6}$ are in principle possible with such a grating fabricated of approximately 400 bilayers with relative error of about $1 \%$.

We have also succeeded in developing in house fabrication of echellette substrates with super smooth etched surfaces. The fabrication process has been optimized by varying such etching parameters as the concentration and temperature of $\mathrm{KOH}$ mix, the etching time, the mechanical interfusion, and the ultra-sonic excitation, as well as the azimuthal disorientation of (111) Si wafer and lithographic mask, or the concentration of oxygen in the Si wafer. The figure of merit for the optimization is surface roughness, and that has been achieved as small as $3 \AA$ (rms).

We are in the progress of testing the etched substrates with scanning electron, interferometric, and atomic force microscopes, and applying different multilayer coatings on the echellette substrate. 


\section{Chemical Sciences Division}

\section{Synthetic and Electrochemical Approaches to Metal-Metal Bonds in Actinides}

Principal Investigator: John Arnold

\section{Project Description}

Understanding the chemistry and, in particular the bonding in actinide compounds is of fundamental importance to advance research in the areas of nuclear weapons and fuel cycles as well as the management of radioactive waste and related environmental cleanup. Indeed, it is from the design and development of processes for large-scale separation and recovery of radioisotopes that much of our knowledge of the chemical behavior of these elements has been derived. We believe that to fully understand the electronic and thermodynamic properties of actinide systems, a thorough assessment of the type and strength of bonding in actinide-ligand, actinide-actinide, and actinide-metal linkages is required. Numerous studies on the former have involved organometallic systems; the removal of aqueous media removes the complicating factors of metal hydrolysis and solvent exchange, and provides more direct comparison of chemical reactivity and structural properties derived from the metal, its oxidation state and ligand type; indeed organometallic systems which feature a variety of metal-ligand multiple bonds have been observed, key examples including organoimido, phosphinidene, and sulfide systems, and recent progress in the molecular chemistry of the actinide elements has led to many notable advances including the isolation of the bis-imido analog of the important uranyl moiety.

\section{Accomplishments}

Our initial attempts are focusing on using bulky terphenyl ligands $\left(\mathrm{C}_{6} \mathrm{H}_{3}-2,6\left(\mathrm{C}_{6} \mathrm{H}_{3}-2,6-\right.\right.$ $\left.\left.\operatorname{Pr}_{2}^{\mathrm{i}}\right)_{2}, \mathrm{Ar}^{\prime}\right)$, in the form of both the lithium salt and as the Grignard reagent, in combination with $\mathrm{U}(\mathrm{III})$ and $\mathrm{U}(\mathrm{IV})$ salts. In the case of the reaction of $\mathrm{UO}_{2} \mathrm{Cl}_{2}(\mathrm{thf})_{3}$ with two equivalents of $\mathrm{Ar}^{\prime} \mathrm{MgBr}$, initial ${ }^{1} \mathrm{H} \mathrm{NMR}$ data suggest the formation of a bis-aryl uranyl complex; further characterization is currently underway. We also began looking at the use of N-based terphenyl ligands such as $\mathrm{Ar}^{*} \mathrm{NH}_{2}\left(\mathrm{C}_{6} \mathrm{H}_{3}-2,4\left(\mathrm{C}_{6} \mathrm{H}_{2}-2,4,6-\mathrm{Me}_{3}\right)_{2}\right.$, $\left.\mathrm{Ar}^{*}\right)$ and in order to gain insight in the potential bonding modes attainable, the less sterically hindered $\left[\left(\mathrm{SiMe}_{3}\right) \mathrm{N}\left(\mathrm{C}_{6} \mathrm{H}_{3}-2,6-\mathrm{Pr}_{2}{ }_{2}\right)\right] \mathrm{Li}$. Our initial reactions proved successful, leading to the isolation and characterization of $\left[\left(\mathrm{SiMe}_{3}\right) \mathrm{N}\left(\mathrm{C}_{6} \mathrm{H}_{3}-2,6-\mathrm{Pr}_{2}^{\mathrm{i}}\right)\right]_{2} \mathrm{UCl}_{2}$, $\left[\left(\mathrm{SiMe}_{3}\right) \mathrm{N}\left(\mathrm{C}_{6} \mathrm{H}_{3}-2,6-\mathrm{Pr}_{2}^{\mathrm{i}}\right)\right]_{2} \mathrm{UI}_{2}$ (from the disproportionation of the $\mathrm{UI}_{3}(\text { thf })_{4}$ starting material $)$ and $\left[\left(\mathrm{SiMe}_{3}\right) \mathrm{N}\left(\mathrm{C}_{6} \mathrm{H}_{3}-2,6-\mathrm{Pr}_{2}^{\mathrm{i}}\right)\right]_{2} \mathrm{ThCl}_{3} \mathrm{Li}(\text { thf })_{2}$. Subsequent reactions of these compounds with a range of alkyl and aryl reagents have yielded a tractable product from the reaction of two equivalents of mesityl lithium with $\left[\left(\mathrm{SiMe}_{3}\right) \mathrm{N}\left(\mathrm{C}_{6} \mathrm{H}_{3}-2,6-\mathrm{Pr}_{2}^{\mathrm{i}}\right)\right]_{2} \mathrm{UCl}_{2}$. Instead of observing the bis-aryl U(IV) compound, however, we observed intermolecular metallation of a $\beta$-silyl methyl yielding the product $\left[\left(\mathrm{SiMe}_{3}\right) \mathrm{N}\left(\mathrm{C}_{6} \mathrm{H}_{3}-2,6-\mathrm{Pr}_{2}{ }_{2}\right)\right]$ $\left[\left(\mathrm{SiMe}_{2}\left(\mathrm{CH}_{2}\right)\right) \mathrm{N}\left(\mathrm{C}_{6} \mathrm{H}_{3}-2,6-\mathrm{Pr}_{2}^{\mathrm{i}}\right)\right] \mathrm{UCl}$. We have also started work on the preparation of complexes featuring interactions between the heavier Group 16 elements (Se, Te) and U and Th. Further studies with these ligands include extending the scope of the reactions to encompass the +3 and +4 oxidation states. In addition to further synthetic work and electrochemical studies on these new compounds, we plan to extend our use of computational methods as both an interpretive and predictional aid to this project. 


\title{
Conversion of Glycerol and Aromatic Compounds from Biomass to Major 3- and 6- Carbon Industrial Organic Compounds
}

\author{
Principal Investigators: Robert G. Bergman, Jonathan A. Ellman
}

\section{Project description}

As the production of biodiesel increases, an effective conversion of glycerol (alternative name: glycerin), a major byproduct in the production of this renewable fuel source, to useful substances is becoming increasingly important. The objective of this project is the efficient and practical conversion of glycerol into synthetically useful three-carbon industrial chemicals. The key for approaching this goal is exploring rational synthetic routes and undertake thorough investigations on the mechanisms of the transformations that are successful.

We have focused on proton-catalyzed reactions, as glycerol is known to undergo dehydration in the presence of acidic substances. Our aim is understanding the fundamentals of these transformations under mild conditions in order to optimize them and reduce formation of undesired byproducts. The mechanistic studies of glycerol conversion, as a simple model molecule for carbohydrates, would also contribute towards the development of other biomass conversions.

\section{Accomplishments}

The main accomplishment of this project was the optimization of the formic acidmediated conversion of glycerol into allyl alcohol with substantially improved yield and selectivity. We have also succeeded in establishing an unpredicted mechanism for this reaction by deuterium labeling experiments. Our results support a mechanism involving formation of a critical transient intermediate, and suggests that the reaction might take place through a potentially isolable orthoester. That intermediate would rearrange at high temperature, resulting in deoxygenation of two vicinal hydroxy groups (syn elimination from the cyclic derivative) giving the diene and releasing an acid and carbon dioxide. We are in the process of confirming this mechanism and exploring suitable conditions for the experimental determination of reaction rates.

In addition, we have carried out extensive exploration of the acid-catalyzed conversion of glycerol into acrolein using inexpensive Brönsted acids. Some of our results show clean conversion to acrolein. Our aim is to understand the fundamentals of this transformation in order to optimize it and reduce formation of undesired byproducts. We have initiated an investigation on the solution phase mechanism of the conversion of glycerol to acrolein. We have developed an independent route to a key cation and have found that it leads to acrolein under carefully controlled conditions. This method of cation generation will enable us to carefully study the reaction mechanism and should provide valuable information useful in the design of more efficient and selective methods for glycerin to acrolein conversion. 


\title{
Chemical reactions at liquid/vapor interfaces probed by photoemission spectroscopy
}

\author{
Principal Investigators: Hendrik Bluhm and Kevin R. Wilson
}

\section{Project Description}

The goal of this project is to build a novel instrument for the investigation of chemical reactions at liquid/vapor interfaces under atmospherically and environmentally relevant conditions. The properties of liquid/vapor interfaces strongly influence heterogeneous processes in atmospheric and environmental chemistry. In particular aqueous aerosols catalyze many heterogeneous reactions in the troposphere and function as both sinks and sources for atmospheric trace gases. Up to now little is known about the concentration of solution phase species at the liquid/vapor interface, which can significantly differ from the bulk solution concentration and is an important quantity in the modeling of heterogeneous reactions at liquid/vapor interfaces. A direct measurement of the liquid/vapor interface under atmospherically and environmentally relevant conditions is difficult due to the lack of quantitative experimental techniques that are surface-sensitive, chemically specific and can operate at elevated pressures in the Torr range. By combining synchrotron-based ambient pressure photoemission spectroscopy (APPES) and a liquid droplet train into a single experimental setup we will address this need and provide quantitative information about the chemical nature of the liquid/vapor interface under varying conditions of bulk concentration and temperature, as well as gas phase composition and pressure.

We have planned our experiments in two stages. In the first phase we have implemented a droplet train generator in the ambient pressure photoemission endstation at beamline 11.0.2 at the Advanced Light Source. This setup allows us to optimize the experimental parameters and, in addition, to perform first experiments on solution/vapor interfaces. In the second phase we are building a dedicated machine that will allow us to vary the interaction time between droplets and gas molecules. This will enable us to investigate heterogeneous reactions that have great relevance to atmospheric chemistry but are currently incompatible with conventional ultra-high vacuum systems (e.g., $\mathrm{HNO}_{3}, \mathrm{H}_{2} \mathrm{SO}_{4}$, and $\mathrm{HCl}$ ).

\section{Accomplishments}

In the first stage of this project we have successfully implemented a droplet train generator in the APPES endstation at beamline 11.0.2. The train of droplets is positioned close to the entrance aperture of the electrostatic lens system of the APPES endstation where incident photons from beamline 11.0.2 irradiate the droplets in front of the aperture, and the resulting electrons are collected by the differentially pumped lens system and probed using the hemispherical analyzer.

To test the feasibility of our approach we have conducted a number of proof-of-concept experiments on various liquid/vapor interfaces, including aqueous salt and alcohol solutions. One example is the investigation of the relative concentration of methanol and water molecules at the liquid/vapor interface of a $0.6 \mathrm{M}$ aqueous methanol solution in equilibrium with its vapor (in this example $\mathrm{p}=2.5$ Torr at $\mathrm{T}=-25^{\circ} \mathrm{C}$ ). We have found that the concentration of methanol directly at the liquid/vapor interface is enhanced by a factor of $\sim 3$ compared to the concentration in the bulk solution. This example shows that our combined droplet train/ambient pressure photoemission spectrometer allows us to directly interrogate the chemical composition of liquid/vapor interfaces under ambient conditions of temperature and pressure. 


\author{
Properties of Ionic Liquids \\ Principal Investigator: John M. Prausnitz
}

\title{
Project Description
}

Ionic liquids are salts that have low melting points, near or below ambient temperature. Ionic liquids typically contain large organic cations and organic or inorganic anions. These liquids are stable to high temperatures, have high electric conductivity, often have low toxicities and exhibit essentially zero vapor pressures at normal temperatures. Ionic liquids are therefore useful as "green" solvents for separation operations (e.g., extraction and absorption), as media for chemical and biochemical reactions, and for electrochemical devices like fuel cells and lithium batteries. Although ionic liquids have been known for many years, the available experimental data base for their properties is small. The goal of this research is to synthesize new ionic liquids and to measure some of their properties such as melting point, thermal stability, viscosity, electrical conductivity, phase equilibria with water, glass-transition temperature and toxicity. Attention is also given to distribution coefficients for some offensive cations between an ionic liquid and a wastewater.

\section{Accomplishments}

Approximately 23 new ionic liquids were synthesized with special attention to those liquids that contain pyridinium, pyrrolidinium, and piperidinium cations. Several taskspecific ionic liquids were synthesized for extraction of selected cations from water. Measurements were made to determine melting and glass-transition temperatures and thermal stabilities (up to about $300^{\circ} \mathrm{C}$ ), viscosities, electric conductivities with and without dissolved lithium salts, mutual solubilities with water (liquid-water and gaseous water in air). Spectroscopic data were obtained to determine polarity and three parameters in the Kamlet-Taft equation commonly used to correlate solubilities and chemical-kinetic rate constants. Further, for a variety of ionic liquids, distribution coefficients were obtained for copper, mercury, silver and palladium salts in water. Chemical reactions at electrode-ionic liquid-electrolyte interfaces were studies by interfacial impedance measurements for a lithium battery. Finally, a perturbed-hardsphere theory was developed to correlate solubilities of common gases in ionic liquids over a wide range of temperature.

Results from the experimental studies serve to identify a suitable ionic liquid for a particular application. For example, ionic liquids containing octylpyridinium cations that exhibit very low solubility in water are highly efficient in removing mercuric ions from wastewater.

As of October 2007, results from this research project have been published in five articles. Two additional articles are now in press and a third is under review. 
New Directions in Radionuclide Materials Characterization Using the ALS Principal Investigator(s): D.K. Shuh, W. W. Lukens, and A. Canning

Project Description

The tradition of innovative experiments with radioactive materials at the ALS will be continued by initiating two new avenues of research that can be explored over a very short timescale yet can produce meaningful results that can be built on for more extensive studies in the future should the investigations yield tangible results as anticipated. The first will be to examine the chemistry of the troublesome fission product technetium (Tc) with soft x-ray synchrotron radiation methods using the experience and the synthetic expertise with Tc materials that has been developed within the Actinide Chemistry Group (ACG) over the past decade. The second will be to inject a theoretical component into the actinide soft X-ray spectromicroscopy effort investigating the fundamental electronic structure of plutonium $(\mathrm{Pu})$ and cerium $(\mathrm{Ce})$ complexes using the scanning transmission x-ray microscope (STXM) to better understand the coordination chemistry of Pu for improved separations.

The soft x-ray investigations will utilize both the Advanced Light Source-Molecular Environmental Science (ALS-MES) Beamline (BL) STXM and the soft X-ray fluorescence capabilities of ALS BL-7.0.1. One priority will be to probe the Tc M-edges while collecting special fluorescence signals since preliminary calculations show that the Tc M-edges would yield valuable Tc speciation information only from fluorescence techniques. To take full advantage of the soft x-ray STXM spectromicroscopy capabilities for the unique investigation of actinide complexes provided by the ALS, a strong component of theory is highly desirable. This theoretical support interaction will be initiated with Computational Research Division (CRD) scientists. CRD scientists are developing new density functional theory (DFT) methods ideal for treating small actinide complexes such as the ones being examined at the ALS by the ACG.

Accomplishments

Magnetite, $\mathrm{Fe}_{3} \mathrm{O}_{4}$, is a possible specialized host material for the long term immobilization of separated Tc from nuclear wastes. Magnetite was synthesized with a $10 \%$ Tc content in which the Tc stoichiometrically substitutes for Fe. Spectromicroscopy of the Tc-doped magnetite was performed with the STXM on the ALS-MES BL. Near-edge X-ray absorption fine structure (NEXAFS) experiments were conducted at the O K-edge and Fe L-edge. Comparison of the Tcdoped magnetite NEXAFS spectra to that from magnetite showed little change in the overall electronic structure. The normal contrast x-ray images of the Tc-doped magnetite particles were obtained on the $35 \mathrm{~nm}$ scale, which are the first micrographs from this material, and showed interesting morphology. The Tc M-edge fluorescence investigations of the Tc-doped magnetite at were complicated by detector performance issues and could not be successfully completed.

The initial studies of the $\mathrm{Ce}$ and $\mathrm{Pu}$ maltol complexes, for which the experimental crystal structures have been determined, have been performed using first-principles electronic structure codes based on density functional theory. In particular, for the Ce maltol complex, the energy levels and density of states of the different valence electrons have been obtained, which can be compared to the experimental NEXAFS measurements. The theoretical predications and the experimental results are in reasonable agreement. However, the calculations with Pu proved more difficult and have some convergence problems due to the partially filled $\mathrm{f}$ band. Other theoretical methods and potentials for Pu that have better convergence properties were explored. 


\section{A New Approach for the Catalytic Conversion of Methane and Other Inert Hydrocarbons Principal Investigator(s): T. Don Tilley}

\section{Project Description}

The selective, catalytic functionalization of saturated hydrocarbons represents one of the most important challenges in chemical research. While some progress has been made, there are very few processes that allow conversion of the cheapest and most abundant hydrocarbon, methane. Intense interest in this topic has led to many important advances including the discovery of several mechanisms by which transition metal species react with unactivated $\mathrm{C}-\mathrm{H}$ bonds. While these studies have revealed a number of interesting stoichiometric transformations, there have been significantly fewer reports describing selective conversions of alkanes via homogeneous catalysis. Methane is a particularly attractive substrate for such conversions since it is cheap and readily available, and represents a potentially useful reagent for the incorporation of methyl groups into molecular structures.

For some years, we have been studying $\sigma$-bond metathesis (the concerted exchange of substituents between two $\sigma$-bonds) as a fundamental process for bond activations, in the context of metal-mediated polymerization chemistry. Studies on the interactions of silanes with $\mathrm{d}^{0}$ metal complexes revealed several pathways for the activation of $\mathrm{Si}-\mathrm{H}$ and $\mathrm{Si}-\mathrm{C}$ bonds via $\sigma$-bond metathesis. This rich reaction chemistry suggested that similar activation steps might be used in catalytic hydrocarbon functionalizations, given appropriately active and selective catalysts. In preliminary tests of this concept, we observed the first catalytic processes that allow conversion of methane to other products via $\sigma$-bond metathesis with $\mathrm{Cp}_{2}{ }_{2} \mathrm{ScMe}\left(\mathrm{Cp}^{*}=\mathrm{C}_{5} \mathrm{Me}_{5}\right)$ as catalyst. These observations raised the possibility for development of homogeneous catalytic processes that utilize methane as a cheap and convenient methylating reagent. The aim of this project was to utilize this chemistry in the development of more efficient catalysts for methane conversions.

\section{Accomplishments}

Efforts in this program have centered on exploration of systems related to the scandium one described above, to learn as much as possible concerning the influence of ancillary ligands, reaction conditions, and substrate variations. It is clear that a soft, strongly donating ligand (e.g., $\left.\mathrm{Cp}^{*}\right)$ supports $\sigma$-bond metathesis reactivity, and variations in the structure of such ligands should reveal factors that influence reactivity in this chemistry. Since electron-rich, anionic $\pi$ systems are generally good candidates, we chose to target the synthesis and development of the permethylfluorenyl ligand $\left(\mathrm{C}_{13} \mathrm{Me}_{9}, \mathrm{Flu}^{*}\right)$. Due to its size and electronic properties, this ligand has the potential for promoting $\sigma$-bond metathesis reactivity and selectivity in bond activations.

The development of a synthetic route to octa- and nona-methylfluorene was accomplished and these compounds were used to prepare mixed-ligand ruthenocene complexes. Structural studies demonstrated that Flu* is $\eta^{5}$-coordinated to the metal center, and initial electrochemical studies of the ruthenium compounds revealed that Flu* is more electron-donating than the $\mathrm{Cp}^{*}$ ligand. We also prepared a family of Flu*-containing zirconium and hafnium compounds, and IR spectra of $\operatorname{Zr}$ (II) dicarbonyl derivatives demonstrated the very electron-donating nature of these highly methylated fluorenyl ligands. Attempts to prepare a zirconium dihydride derivatives led to the isolation of a complex derived from hydride transfer from the metal to the Flu* ligand, and hydride complexes in this system participate in a wide variety of reactions such as hydrogenation, olefin dimerization, and $\mathrm{C}-\mathrm{H}$ activation of pyridine derivatives. Methane activations have not been observed in this system, but these are expected to result from analogous group 3 derivatives. 


\author{
Versatile Mini-Scanning Transmission X-ray Microscope (mSTXM) \\ Principal Investigator(s): Tolek Tyliszczak and David K. Shuh
}

Project Description

The objective of this project was to develop a new Scanning Transmission X-ray Microscope (STXM) for the Advanced Light Source (ALS). There was a timely opportunity to design and implement a compact, stand-alone, prototype STXM instrument that will provide additional STXM capacity for scientific investigations utilizing existing beamlines at synchrotron radiation sources such as the ALS without the high opportunity cost. The research team that designed and built the existing ALS STXMs was to develop a compact STXM microscope which can be fabricated at a lower cost than existing STXMs, while at the same time providing the experimental versatility with nearly all of the current STXM performance characteristics but at the same time allow for some additional experiments. The new microscope, the mini-STXM (mSTXM), was envisioned as a portable end station which could be attached to several existing ALS beamlines for experiments and will also serve as a developmental test platform for future STXM innovations at the ALS.

There are three major technical design challenges which have to be overcome in an innovative and economical manner to successfully realize the portable mSTXM instrument. Very low relative vibrations, precise and fast sample scanning and efficient and time resolved photon detection are those challenges. The approach to meet these technical requirements and the overall goals for the mSTXM, is the universal utilization of compact, state-of-the-art piezoelectric scanning/positioning stages.

\title{
Accomplishments
}

One fundamental part of the initial mSTXM conceptual design was to employ a long range piezo flexure as the sample scanning stage. Intensive experimental studies with a demonstration nanocube unit showed that this piezoelectric stage had too much mechanical noise and therefore can not be used as a basis for the mSTXM. An extensive search for an alternative solution led us to re-examine the entire overall conceptual design. This has resulted in a new, updated design of an even more compact mSTXM, the mSTXMII, that has performance specifications akin to existing large STXMs but comes with an accompanying increase in the estimated per unit fabrication cost.

The new mSTXMII design uses ultra-high vacuum (UHV) compatible linear positioning and scanning stages obtained from a commercial manufacturer. These stages are commonly used in this manufacturer's atomic force microscopes and according to the specifications, should provide mechanical noise in the range of few $\mathrm{nm}$. This should ensure that the mSTXMII resolution will be limited by the zone plate even when the best foreseeable zone plates become available. Furthermore, the new mSTXMII design eliminates the energy range limitation inherent in the original $\mathrm{mSTXM}$ design by using a linear positioning zone plate stage with a laser interferometer.

The mSTXMII has been built and commissioning conducted. During initial commissioning some potential problems have been discovered which may impair the final performance of the microscope. The problems stem from inadequate precision of the positioning stages. Unfortunately, there were no other commercial alternatives to these stages which operate in a UHV environment and have very low vibrations. During further commissioning, attempts to rectify some of these problems will be continued. Even with these limitations, the innovative design opens doors to new experiments such as low temperature time-resolved magnetization measurements with opaque samples using the electron yield signal. This microscope will also help in very important measurements of magnetization dynamics by spin injection. The samples are typically $100 \mathrm{~nm} \times 100 \mathrm{~nm}$ in size and require multi-hour exposure, which in the existing STXM leads to a thick carbon layer being deposited on the sample which partially obscures the signal. 


\section{Computing Sciences}

A Structured, Adaptive Mesh Refinement Method for Multiphase Reactive Transport in Groundwater

Principle Investigator: John B. Bell

\section{Project Description}

High-fidelity simulations of groundwater flow have the potential for providing valuable insights into long-term fate of contaminants. However, realizing this potential presents significant computational challenges. We propose to perform a proof of concept study to demonstrate the effectiveness of adaptive mesh refinement (AMR) for reactive subsurface transport. The project will develop a basic flow module for modeling multiphase, multicomponent non-isothermal flow with reactions. This methodology will include second-order discretization methods that provide a robust and accurate treatment of transport processes and the coupling of that integration scheme to parallel AMR methodology. The overall goal is to validate and demonstrate the effectiveness of this approach.

The flow of subsurface fluids is governed by conservation laws for chemical species and energy, including the effects of geochemistry and phase behavior, together with phase velocities given by Darcy's law. Our approach is based on a sequential formulation of the porous media flow equations. In this approach we first solve a pressure equation. We then solve the multicomponent conservation and energy equations using a fractional flow formulation. The overall integration approach is combined with AMR methods that include support for large distributed multiprocessors. Specialized linear solver must also be developed to address the anisotropic meshes typically used for subsurface flow problems.

\section{Accomplishments}

We have made two significant accomplishments in this project. First, we have performed a mathematical analysis of two-phase, multicomponent, non-isothermal porous media flow equations. This analysis shows that the component conservation and energy equations are hyperbolic so that a total velocity splitting approach can be applied to this type of flow. The results of this analysis also form the basis for the development of highresolution simulation methodologies for these types of systems. Our results show that the structure of the system is determined by an isenthalpic flash calculation in which the phase behavior is characterized by maximizing the entropy at given composition and total enthalpy.

Our second accomplishment has been the development of a framework for AMR for subsurface flow. The framework currently provides support for incompressible systems on isotropic meshes and has been tested on some simple single-phase, multicomponent problems. The current approach is second-order in space and time and supports subcycling in time so that finer spatial grids are advanced with smaller time steps. Specialized synchronization algorithms are used so that the integration of the finer levels of refinement does not require global elliptic solves over the entire grid hierarchy. 
On-Demand Overlays for Scientific Applications

Principal Investigator(s): Karlo Berket, Chin Guok

\section{Project Description}

The practice of modern science is increasingly dominated by large-scale collaborations of multi-disciplinary teams integrating results from both simulation and observation. Scientific computations and collaborations increasingly rely on the network to provide high-speed data transfer, dissemination of results, access to instruments, support for computational steering, etc. ESnet is establishing a science data network to serve large point-to-point transfers, a common case for applications such as gridFTP. It is also possible to use this network to serve data dissemination from a single sender to multiple receivers, e.g. for monitoring the Large Hadron Collider.

Application overlay networks have been effective in providing data aggregation and dissemination services in the general Internet. An application overlay network creates a virtual application-layer topology that sits on top of the physical network. This application-layer topology can be dynamically configured either through a centralized decision point or a distributed algorithm. This enables the application overlays to optimize network utilization for the application data traffic. This is in contrast to current MultiProtocol Label Switching (MPLS) approaches that always choose a single "best" path. The majority of overlay algorithms and protocols have been designed to work at the application layer of the networking stack. We believe that the existing work at the application layer can be applied to produce algorithms and protocols that make efficient and effective use of resources at lower layers of the network stack. We also believe that we can leverage the research done on QoS routing via multiple paths and recent work on Automatic Multicast Tunneling (AMT).

\section{Accomplishments}

The goal in this project has been to perform the research and development necessary to understand and enable the building of a framework for creating on-demand overlays for scientific applications. A critical goal of this work is to enable efficient and effective use of the available on-demand network resources. In this project we are focused on the two important scientific use-cases: creation and use of redundant paths for bulk traffic and creation and use of a dissemination tree for one to many traffic. Specifically, we researched and developed methods for instantiating on-demand virtual overlays on the physical ESnet network, and the understanding of algorithms tuned to the application requirements and underlying network characteristics.

This past year, our work focused primarily on the point-to-point bulk data transfer use case. We developed a prototype overlay for transmitting bulk data over multiple paths to a single destination. GridFTP was used as the bulk transfer application on the end hosts and we conducted a range of tests to understand the properties of the transfer. Our tests showed the value of the multi-path overlay but illuminated a weakness in gridFTP that prevented it from using the multiple paths effectively. Our results have been documented in a paper and submitted for publication. An important step in this research is developing a capability to instantiate multiple paths on-demand. We began work this year on algorithm development to enable path selection based on ESNet data from the OSCARS project. OSCARS provides on-demand circuit reservation in ESNet. 


\title{
Cryptographic Foundations for New Generation Distributed Systems
}

\author{
Principal Investigator: Olivier Chevassut, Abdelilah Essiari
}

\section{Description}

New generation distributed systems (e.g., peer-to-peer and Grid middleware) are networking technologies that enable complex interactions among computational and data resources. These distributed communication middleware employ complex message exchange models (e.g., one-way, asynchronous, bilateral, multi-node paths) that cannot be supported by existing security solutions. If these distributed systems are to be successful and widely deployed in production computing environments the systems need to be enhanced with the adequate cryptographic mechanisms. While the usual security notions are still valid, generation distributed systems require new cryptographic foundations. This project provided the cryptographic foundations and technologies for these distributed systems.

\section{Accomplishments}

This project defined the requirements of authentication and key exchange for the nextgeneration distributed applications. Technologies were developed to allow for the authentication of two or more parties using short secret keys called passwords. It extended the technologies to allow cryptographically secure authentication and key exchange involving low-power mobile devices. Examples of these devices are PDAs and laptops. Corruption of users' password was also taken into account by adding one-time password features to the cryptographic technologies.

A second requirement of a security infrastructure is to protect against servers' database corruptions which occur when hackers penetrate an enterprise (or laboratory) security perimeter and access the database storing all the users' passwords. The hackers can then run a dictionary attack and recover the users' names and passwords. Preventing this type of attacks involves adding verifier-based features to authenticated key exchange technologies. This project tackled this issue as well.

The issue of patents in the area of encryption technologies is often a major factor preventing the wide use of a cryptographic technology. The present project looked at this difficult and controversial issue in order to see how the cryptographic technologies developed in the context of this project can be freely implemented and distributed. Promising avenues were identified, and preliminary analysis and modifications of the cryptographic technologies were undertaken and published.

Specifically, in this last year we studied the problem of authentication and key exchange to provide solid cryptographic foundations to secure next generation services communications. 
Software Application Infrastructure For Efficiently Managing Large-Scale Computational Biology Experiments

Principal Investigator(s): David Konerding

\section{Project Description}

The purpose of this project is to enable large-scale computational experiments being carried out by the Brenner laboratory which are designed to increase our understanding of gene regulation and genetic diseases in humans. These experiments will elucidate nonsense-mediated decay, a eukaryotic mRNA transcript surveillance mechanism which ensures that improperly spliced transcripts are degraded before being translated into protein. The Brenner laboratory performed a combination of computational screens and lab experiments showing that NMD plays an important role in several disease-related genes, including Calpain-10 (susceptibility to Type-II diabetes in several populations), Presenilin1 (linked to Alzheimer's disease susceptibility) and lymphocyte associated receptor of death (associated with lymphoid malignancies). The list of diseases associated with NMD is growing rapidly. However, current work only scratches the surface of detecting NMD candidates. We need a computation-based experimental infrastructure (C-BEI) so we can make a number of important methodological improvements to the NMD detection pipeline, significantly extending its sensitivity and specificity. With this improved pipeline, significantly more sophisticated experiments can be carried out.

We will enhance our prior work on C-BEI with a Parameter Mesh Refinement (PMR) method, which has the potential to dramatically reduce the number of computations required to find the maxima. PMR samples the performance of an algorithm by trying an initial random set of parameters, then fitting a multidimensional surface to the output function of the algorithm. The interpolated maxima predicted by the surface fitting will then be sampled to progressively refine the estimated output function surface until a termination threshold is reached. We will develop the PMR technique and implement a workflow component, which will allow scientists to easily convert their existing parameter sweeps into PMR sweeps.

\section{Accomplishments}

We developed Python interfaces to two surface fitting libraries, OpenRM (www.openrm.org) and WildMagic (www.geometrictools.com) to implement the surface fitting functionality and ran a large collection of full parameter space exploration using the SSEARCH sequence search algorithm. We then implemented our algorithm for sampling the surface and attempted to find the global maximum. However, we found that the underlying data that we were surface fitting was not smooth enough, and had too many unpredictable maxima, meaning the algorithm was not able to reliably produce a valuable computational speedup relative to full parameter space exploration. 


\title{
Integrated Microbial Community Genomes Data Management System
}

\author{
Principal Investigators: Victor M. Markowitz, Natalia N. Ivanova, \\ Nikos C. Kyrpides, and Phil Hugenholtz
}

Project Description

Studies of the collective genomes (also known as metagenomes) of environmental microbial communities (also known as microbiomes) are expected to lead to advances in environmental cleanup, agriculture, industrial processes, and alternative energy production. Similarly, studies of the metagenomes of human microbiomes will provide new insights into variations of microbial populations associated with the human body in health and disease, and will lead to the development of new treatment strategies.

The purpose of this project is to develop metagenome data management and analysis tools in the context of integrated microbial genome and metagenome data. These tools will provide support for the integration and interpretation of metagenome sequence data and sample metadata; functional annotation and metabolic reconstruction for metagenome sequence data; comparative data analysis and visualization of metagenome sequence data in the context of sample metadata and isolate microbial genome sequence data. Metagenome data analysis tools These tools will allow addressing fundamental biological questions, such as how are functions distributed within a community and how does a community respond in both the near and long term to environmental changes as well as applied environmental questions.

Accomplishments

In FY 2007, new metagenome data management and analysis tools were gradually developed and provided to scientists for analyzing environmental metagenomes. A substantially extended metagenome data warehouse was released to the scientific community on January $22^{\text {nd }} 2007$ as IMG/M (http://img.jgi.doe.gov/m). IMG/M includes a total of 2,301 genomes consisting of 595 bacterial, 32 archaeal, 13 eukaryotic genomes, and 1,661 viruses, integrated with metagenome sequences generated from several environmental microbial community samples including an acid mine drainage biofilm, an agricultural soil sample, and two biological phosphorus removing sludge samples, two human distal gut samples, a gutless marine worm sample, and obese and lean mouse gut samples.

Data processing tools have been developed for handling an increasing number of metagenome data sets generated using 454 Life Sciences pyrosequencing technology. Additional metagenome data analysis tools have been developed to support the exploration and comparative analysis of metagenomes in the context of isolate microbial genomes and other metagenomes. Tools for comparing the functional capabilities of metagenomes have been extended with tests for estimating the statistical significance of the observed differences.

IMG/M has proved valuable in completing the analysis of the hydrogen-producing microbiome colonizing the termite hindgut. The recently published mouse gut metagenome data set has been included into IMG/M, thus providing a unique opportunity to compare allopatric populations of the same bacterial species inhabiting drastically different environments in terms of their gene content and metabolic capabilities. 


\section{Enhancing Commodity Scalar Processors with Vector Components for Increased Scientific Productivity \\ PIs: Leonid Oliker, John Shalf, Katherine Yelick, William T. Kramer}

Project description:

The increasing gap between processor and memory speeds has become a wellknown problem in computer architecture. Furthermore, techniques designed to hide memory latencies, such as out-of-order superscalar instruction processing, speculative execution, multithreading, and stream prefetching engines, may actually increase the memory bandwidth requirements. This so-called "memory wall" is one of the primary reasons many high performance applications run well below the peak arithmetic performance of the underlying machine.

The goal of our research is to address these challenges by exploring the opportunities for improved scientific productivity on two fronts. First we examine the potential of combining vector processing features with commodity processors. In particular, we are identifying the minimum set of hardware features required to see the benefits of vectorization without compromising the cost-effectiveness of commodity processors for general purpose processing. Next, our work explores alternative caching strategies, such as software controlled memory architectures, with the goal of leveraging these techniques to improve scientific productivity.

Accomplishments:

We developed the Virtual Vector Architecture (ViVA), which combines the memory semantics of vector computers with a software-controlled scratchpad memory in order to provide a more effective and practical approach to latency hiding. ViVA offers the hardware simplicity of software-controlled memory hardware implementations, with familiar vector semantics that are amenable to existing vectorizing compiler technology. A unique advantage of this approach is its ability to coexist with conventional cache hierarchies. ViVA requires minimal changes to the core design and could thus be easily integrated with conventional processor cores. Our work demonstrate ViVA's capabilities using IBM's Mambo cycle-accurate full-system simulator. Results show that ViVA offers superior performance compared to a microprocessor using conventional hardware and software prefetch strategies.

Additionally, we conducted extensive analysis of important scientific kernel performance across a variety of leading multicore platforms. Results show that the Cell processor offered the highest raw performance and power efficiency for our evaluated numerical kernels, despite having peak double-precision performance and memory bandwidth that is comparable to other platforms in our study. The key architectural feature of Cell is explicit software control of data movement between the local store(cache) and main memory. However, this impressive computational efficiency comes with a high price - a difficult programming environment that is a major departure from conventional programming. Nonetheless, these performance disparities point to the deficiencies of existing automatically-managed cache hierarchies, even for architectures with sophisticated hardware and software prefetch capabilities. Thus there is considerable room for improvements in the latency tolerance techniques of microprocessor core designs. 


\section{Advanced Computational Tools for Electric Power Systems}

Principal Investigators: Ali Pinar, Bernard Lesieutre, and Juan Meza

\section{Project Description:}

As society's dependence on engineered networks such as the electric power, communication networks, and the Internet expand, the need for secure and reliable operational standards for these systems becomes vital for the economic, energy and national security of our country. The August 14, 2003 blackout in the Northeast depicted the possible catastrophic consequences of a few broken power lines. This blackout affected 50 million people in the Northeast, its estimated cost in the U.S. was $\$ 4-10$ billion, and it took as many as 4 days to recover.

The need for improved operational and reliability standards for the electric power system is incontestable. Such efforts however, are hindered by the lack of computational tools that can provide better modeling capability and system understanding. We claim that the improved reliability standards for electric power systems will come with advanced computational tools and high performance computing, and LBNL as a renowned institution in optimization and environmental energy research has a great opportunity to step up to be the center of research in computational electric power systems.

\section{Accomplishments:}

Our work in the previous years of this project, focused on designing mathematical models for the vulnerability analysis problem, where we seek a small group of lines, removal of which will cause a significant disturbance to the system. This year we focused on designing algorithms to solve the associated optimization problems for our mathematical models. Our first model leads to a mixed integer nonlinear programming (MINLP) problem, where nonlinearity comes from the power flow equations and the blackout severity metric that we have developed, while the integer variables correspond to identifying broken power lines. This leads to an inherently difficult problem to solve. While the continuous relaxation technique that removes the integrality constraints on the line parameters, worked for small-sized problems, its effectiveness reduced significantly as the problem sizes increased.

As an alternative solution technique, we restricted the power flow model to only active power, by fixing voltages values. This reduced model still leads to a MINLP problem, but our analysis of the structure of a feasible solution identified a special combinatorial property: At a feasible solution to our MINLP formulation, the power grid is divided into two a generation rich region and a load rich region, and the severity of a blackout can be accurately estimated by the load/generation mismatch between the two regions and the capacity of the active edges between these regions. The key new observation here was the correspondence between the Jacobian matrix that represents the nonlinear power flow feasibility and the Laplacian that represents the power grid topology. We took advantage of this observation to reduce the problem to a pure combinatorial problem. Our empirical studies verified the accuracy of our approximation.

We have defined the inhibiting bisection problem, and an integer programming formulation for this NP-complete problem. As the third step we defined the inhibiting bisection problem, which is close to the network inhibition problem, but is polynomial-time solvable, as we have showed. We applied our techniques to benchmark power systems with more than $13 \mathrm{~K}$ nodes and $16 \mathrm{~K}$ lines, where the solution times were only in the order of minutes.

Our results drew attention from other fields, where network models are used and we are investigating applicability of our techniques other areas, such as subsurface flows on fractured rock, biological networks, the transportation network, and data analysis. 


\section{Statistical Feature Modeling for Scientific Data Via Basis Decomposition Principal Investigators: Raquel Romano and Chris Ding}

\section{Project Description}

This project applies machine learning algorithms to problems in scientific data analysis. In contrast with many task-specific scientific data analysis methods, machine learning techniques are data-driven and easily adapted to changing data sets and experimental conditions. Statistical models are built directly from observed and simulated data and then queried in order to answer questions in the domains of radiation biology, astrophysics and climate modeling. Supervised learning techniques are used to detect and quantify phenomena of interest from microscopy and astronomy imagery, e.g., the detection of DNA-repair proteins in high-throughput biology and the recognition of supernovae and other celestial objects in large-scale digital sky surveys. Unsupervised learning algorithms extract structural (e.g., spatial, temporal, spectral) patterns and trends from observed and simulated data, and are useful when the behavior of the signal is due to both deterministic processes and stochastic processes that introduce uncertainty into measured values. Linear and nonlinear decomposition methods are applied to synthetic and observed Type Ia supernovae spectra in order to characterize the spectral features that describe a spectrum's variability due to physical parameters such as age or luminosity. Statistical models of climate simulation data at multiple scales are investigated in order to characterize the spatiotemporal patterns in simulations run under different external forcing scenarios.

\section{Accomplishments}

The first year of the project focused on supervised and unsupervised methods for analyzing protein signals in microscopy imagery. In the second year, we began collaborating with the Nearby Supernova Factory at LBNL to deploy a fully integrated, state-of-the-art classifier, the Support Vector Machine (SVM), into the pipeline that searches $80 \mathrm{MB}$ of imagery per night for early Type Ia supernovae. In the third year, we continued to collaborate with astrophysicists to finish the work on supernova image analysis.

We further investigated data analysis tools for data clustering and dimension reduction. We implemented the affinity propagation algorithm, which was proposed recently by Frey and Dueck (Science, v. 315, pp. 972-976, 2007) for solving the data clustering problem and compared it with the K-medoids method. The preliminary results showed that the Frey-Dueck algorithm typically gave better solutions. We also found that the behavior of the Frey-Dueck algorithm was very similar to that of the K-means clustering method.

To reduce the work required in clustering analysis, we proposed an adaptive dimension reduction approach, which employs K-means clustering technique to generate class labels and uses linear discriminant analysis for subspace selection. Experimental results on real-world datasets indicated that the approach was effective. Finally, we also developed techniques for solving consensus and constrained clustering problems using nonnegative matrix factorization. Consensus clustering refers to the situation in which a number of different (input) clusterings have been obtained for a particular data set and it is desirable to find a single (consensus) clustering that is a good fit in some sense to the existing clusterings. Constrained clustering refers to situations where constraints are known a priori. 


\section{Power Efficiency Metrics for High Performance Computing John Shalf, NERSC Center Division}

\section{Project Description:}

The goal of our effort is to identify appropriate metrics for rigorously comparing the effective computational efficiency of radically different HPC system architectures. Our effort is distinct from existing power efficient computing research activities because it provides a sharper focus on the computational requirements of large-scale scientific problems. To accomplish this, we will use NERSC workload analysis to establish throughput metric based on efficiency on an accurate model of a typical HPC workload, define data collection procedures to establish common metrics for performance for strong and weak scaled scientific problems on different computational platforms, and Benchmark different existing HPC system deployments to refine the benchmarking process. Initially we plan to study factors for uniform power efficiency metrics that will also enable us to provide concrete answers regarding the effectiveness of less-radical approaches to improving power efficiency such as reducing CPU clock rates or reducing DRAM size.

\section{Accomplishments:}

We have worked closely with Erich Strohmaier and the Top500 list maintainers to retarget the list to emphasize power efficiency. This entailed defining a strategy for accurately collecting power efficiency data and incorporating it into the list. We presented our plans at the SC2006 Top500 meeting.

In order to test out our proposed strategy, we collected detailed power efficiency data from a number of microprocessor architectures. We then moved to benchmarking largescale systems, including collecting benchmark and power-efficiency data on the new NERSC flagship computing system - a 20,000 processor 100 Teraflop Cray XT4 (franklin). NERSC provided dedicated time on the Franklin system for our assessment as well as access to secure areas containing the power distribution equipment where we could take reading of power draw under many conditions. The results are summarized in technical report LBNL-64259.

The approach for measuring power efficiency for large-scale systems was then presented to the HPC community at the ISC2007 meeting in Dresden, Germany for public input. We refined our approach, presenting results at IEEE SC2007 Conference.

Finally, the models developed in this LDRD played a central role at the Power Efficiency workshop at SC2007, and have resulted in sustained collaboration with the LBL EERE department. The proceedings of this meeting can be found at (http://esdc.pnl.gov/12 SC07_BOF.stm )

A comprehensive report on all of our activities is in development and has been accepted at the IPDPS workshop on High Performance Power-Aware Computing (HPPAC). This project has accomplished the necessary first steps towards a comprehensive energyefficiency metric that can be used for EPA Energy Efficiency standards such as ServerMetrics but for the HPC domain. 


\title{
Integrated Performance Monitoring: From Application to Workload David Skinner
}

Project Description:

\author{
Lawrence Berkeley National Laboratory
}

Monitoring the performance of scientific computation through passive profiling provides a reasonable middle ground to accommodate the needs of the science users and center managers in getting the most out of large scale scientific compute resources. This LDRD increases the coverage of Integrated Performance Monitoring (IPM) across HPC workloads via a two pronged approach. 1) Increasing the applicability of IPM to the segments of the HPC application space which are currently not covered. 2) Improving the backend infrastructure of IPM so that it can be used for quantitative analysis of the center-wide workload.

These two goals reinforce one another and can be pursued in parallel. They also reinforce the current deployment of IPM at the NERSC center. This LDRD has performed software development and research to ensure that IPM will be available on tomorrow's architectures and applications. It will also enhance the generality of IPM by encouraging funding and collaboration outside the NERSC center.

Accomplishments:

In 2007 we added profiling for both parallel POSIX I/O at scale as well as data transfer profiling of WAN data movement through grid-ftp.

The POSIX I/O profiling extensions to IPM have been implemented and are now in use by HPC researchers on NERSC Linux, Cray, and IBM parallel machines. It was found that the rate at which I/O calls are made is significantly lower than the rate at which MPI function calls are made. As a result we have been able to collect both profiles as well as full traces of several DOE applications. Such profiling reveals the importance of I/O performance in addition to communication and computation. The figure below shows a 256 processor computational cosmology.

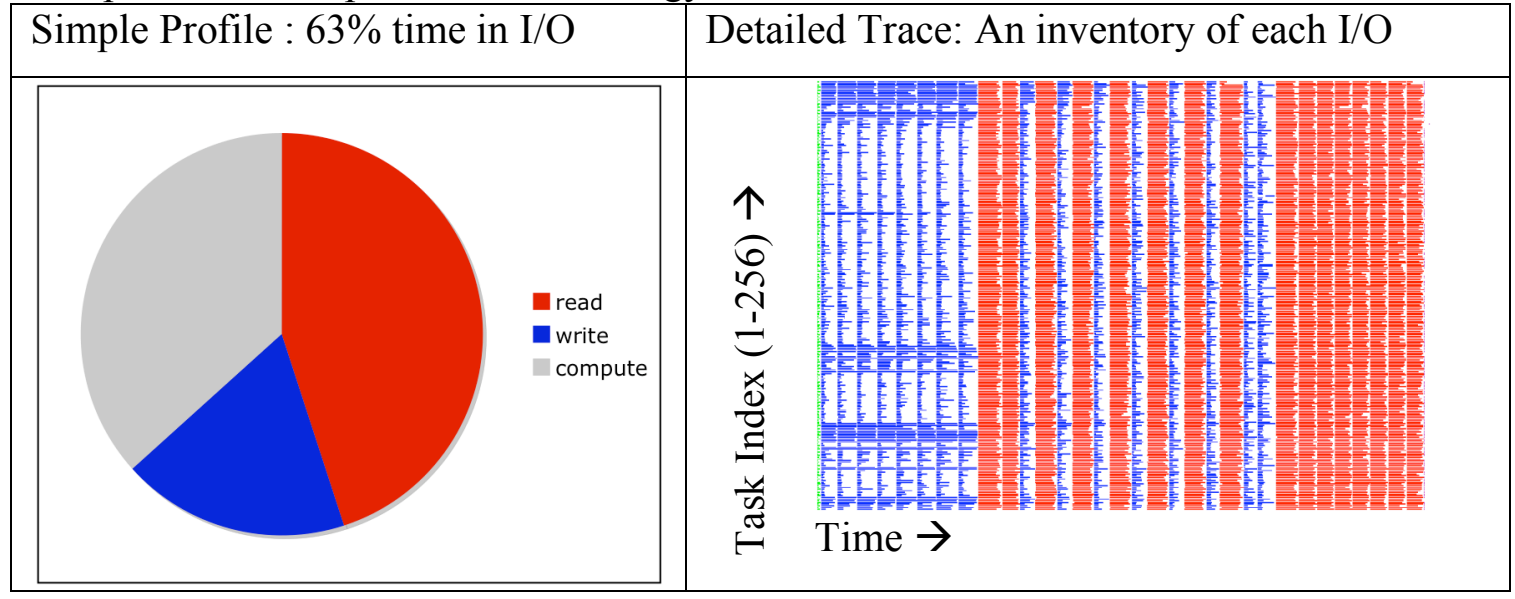

Bandwidth profiles from inter-lab data transfer have also been collected and a workflow which populates a performance database with bandwidths, data sizes, and more detailed information which profiles data being moved through the grid-ftp interface. 
Extended First Order System Least Squares Finite Elements Principal Investigator(s): Jon Wilkening

\section{Project Description}

The purpose of this project is to develop numerical methods in the least squares finite element setting for adjoining special basis functions to the finite element space in order to capture features of the solution which are not accurately represented by the usual basis functions. We intend to apply these methods to problems in solid mechanics involving stress singularities (e.g. crack propagation and grain boundary evolution), and to problems in fluid mechanics involving coupling with elastic or inertial bodies, and in thin channel-like geometries with rough walls.

This project may be divided into three key components. First, the asymptotics of the solution near corners and interface junctions must be understood and a stable way of computing basis functions to represent the singular part of the solution must be devised. Second, these basis functions must be adjoined to the finite element space and an algorithm for computing the stiffness matrix must be developed to avoid the difficulties which are associated with the presence of the singularities. This should be done in the context of interesting physical problems so that the method is of practical use. And third, a strategy for performing an error analysis in the presence of the singular functions must be developed in order to identify the quantities that affect the error and show that the method can be trusted for other application problems which we have not yet explored empirically.

\section{Accomplishments}

We have performed an error analysis of our algorithm for finding canonical systems of Jordan chains for analytic matrix functions. This algorithm lies at the heart of our method for computing stable representations of singular functions describing the asymptotic behavior of the solution near a corner or interface junction. In FY06, we had shown that there is a one to one correspondence between generalized power solutions of the partial differential equation and Jordan chains of an associated analytic matrix function, but we had not yet understood the stability properties of the reduced (matrix) problem. This work was published in June, 2007.

We have also used the techniques we developed to study asymptotic expansions for corner singularities to derive rigorous, a-priori error estimates for Reynolds' lubrication approximation and its higher order corrections in a narrow channel. Instead of solving the equations of fluid mechanics near a corner, we now solve the equations in a thin domain and perform the asymptotic expansion in powers of the aspect ratio $\varepsilon=H / W$. We have derived a nice formula showing how the constants in the error bound depend on the geometry of the channel, which has been an open question for many years. This work was submitted for publication in June, 2007.

We are currently considering the combined problem in which the channel boundary itself has a corner, which is a very interesting synthesis of the two asymptotic regimes studied already. (We are using our X-FOSLS method to study this problem numerically). Finally, we continue to work on the rigorous error estimates of our X-FOSLS method; we believe the tools we used to analyze the lubrication approximation will prove valuable here as well, but this is work in progress. 
Microarray technology for fungal identification: Its utility from the environment to homeland security, biomedicine, and beyond.

Principal Investigator: Gary Andersen

\section{Project Description}

This project will yield biomarker gene sequences from a unique collection of fungi (collected around Chernobyl over 15 years), will provide the nucleus for a web-based database of fungal biomarkers available to other researchers, and will result in the development of a prototype DNA microarray specific for fungal identification. Such a microarray has many uses, from environmental to medical applications and many advantages over current technologies.

The database constructed through this work will be used to select DNA probe sets that will allow the detection and identification, to the level of genus or species, of fungi from any source material. This microarray could greatly advance our knowledge of the role fungi play in important matters such as bioremediation, carbon sequestration and human diseases in addition to providing a monitoring tool for protection against bio-terrorism.

We will sequence variable regions of the large subunit nuclear ribosomal RNA gene and the preceding two internal transcribed spacer (ITS) regions of up to 2000 fungi collected from the Chernobyl Exclusion Zone over the past 15 years. As the entire region cannot be sequenced at one time, two overlapping primer sets will be used to provide a way to concatenate the sequences. The sequences obtained will be used to develop a database similar to our previously developed bacterial biomarker sequence database (Greengenes.lbl.gov), which will be available to other researchers online. The compiled sequences will then be used to select DNA sequences unique to fungal families, genera and species. These DNA sequences will provide the data necessary to construct a prototype microarray for detecting and identifying fungi from collected samples.

\section{Accomplishments}

Our accomplishments have been in three main areas: obtaining DNA sequences from novel fungi isolated from Chernobyl, preparing an online database for environmental mycologists and adapting probe selection software for the design of the microarray for fungi detection.

We have organized sequences for ribosomal genes from the public databases. In addition, we sequenced new fungal biomarker sequences from the Chernobyl Exclusion Zone. In FY'07 we collected an additional full-length $18 \mathrm{~S}$ rRNA genes. Similarly, we have greatly expanded our database of sequences from the D1D2 region of the $28 \mathrm{~S}$ rRNA. For strain-level resolution we have compiled all known ITS sequences, and have chromatograms for nearly half. These three sequence sets were derived from vetting data from multiple studies surveying 2,347 fungal taxa and are now the largest curated compilations in the field. The alignments can be viewed online with restricted access in our pre-release database.

We modified our custom microarray design software to select sets of 25-mer probes for each taxa for sequence-specific detection. Prototype arrays are being manufactured by Affymetrix and will first be tested by monitoring the populations of Candida and the xylose fermenting yeast, Pichia, within the guts of wood eating beetles. This study will demonstrate the utility of the microarray to monitor fungal populations involved in lignocellulose degradation, which is a critical step to DOE's biofuels program. 
Applications of adjoint field methods and time-reversal data processing to inverse problems in electromagnetics, seismics, and ultrasonics

Principle Investigator: James G. Berryman

\section{Project Description:}

Research in complex geophysical imaging situations has shown that one robust method of attacking many imaging and target localization problems confronting geoscientists is the adjoint field method. This method had appeared early in reflection seismic imaging for oil industry applications, in part because the huge size of the data sets precluded any other method. This approach and its generalizations have considerable value in many applications including nonlinear imaging problems in ultrasonics, electromagnetics, and also seismics. Of special relevance, the related method of time-reversal data processing has been developed for both diagnosis and treatment in biomedical problems and, also, for communications in underwater applications, as well as for more traditional ultrasonic imaging in NonDestructive Evaluation (NDE). Time-reversal data processing is itself another example of the successful use of this very general adjoint field method. Two areas of important applications being pursued in the project are therefore ultrasonic/seismic imaging for laboratory/field applications and electromagnetic target location and characterization.

Accomplishments:

In the first year, work on time-reversal analysis targeted at locating and using the information in multi-static data arrays to characterize various targets of interest was completed and published in the journal Inverse Problems. For electromagnetic sensing, targets could be metallic objects hidden in foliage, or buried UXO (unexploded ordnance). Both the eigenvalue number (3 or 6) and character (how their magnitudes decline with distance, for example) permits information to be gathered about objects of interest. Work completed and published includes extensions of the analysis of scattering from spherical targets to scattering from ellipsoidal targets of arbitrary aspect ratio and orientation (collaboration with D. H. Chambers of LLNL).

Work in progress concerns a closely related problem being studied wherein the same sorts of tools will be applied to scattering from nonspherical objects using elastic (seismic/acoustic) waves. Applications of the results to problems in the ocean, earth, and also medicine abound. Extensions are nontrivial because typical work in time-reversal has concentrated on scalar inhomogeneities/scatterers (like the bulk modulus in an acoustic setting). However, the method itself is very robust because it depends mostly on the existence of pertinent Green's functions, and not on the details of the propagation medium, or the targets of interest. The recently published work expands on these ideas to include dipole scatterers (nonuniform density in acoustics). The next step is to include quadrupole scatterers (like inhomogeneous shear modulus in an elastic or seismic setting). This work (in progress) is technically difficult, but conceptually a very natural extension of what has already been accomplished. 


\section{An investigation of the microbial processes involved in a biological fuel cell PI - John D. Coates}

\section{Project Description}

In a microbial fuel cell (MFC) bacteria transfer electrons derived from the catabolism of various alternative compounds onto an electrode (anode). The electrons are subsequently conducted through an electrical circuit towards a cathode in a separate chamber thus generating energy to do work. Chemotrophic biofuel cells are based on the ability of chemotrophic microorganisms to generate reducing equivalents through the oxidation of organic or inorganic chemicals and pass electrons onto an electrode either directly or indirectly through an electron shuttling compound. Many organic and inorganic substrates can be converted into electrical energy in biofuel cells, and the utilization of a given compound is dependent on the metabolic capability of the particular organism on which the fuel cell is based. The goals of this project are to investigate the microbial processes in energy generation in both the anodic and cathodic chambers of an MFC.

\section{Accomplishments:}

A. Research on the anodic chamber. Our studies on MFCs FY 2007 focused on investigating the operation and microbiology associated with thermophilic MFCs operating at $55^{\circ} \mathrm{C}$. Over 100 -day operation, these MFCs were highly stable and achieved a maximum power density of $24 \mathrm{~mW} \cdot \mathrm{m}^{-2}$ and a columbic efficiency of $89 \%$ with acetate as the sole electron donor. In order to characterize the microbial community involved in thermophilic electricity generation, 16S rRNA and rRNA gene were isolated from the electrode and PhyloChip analyses performed. Exploring the changes in the microbial community over time revealed an increase in relative abundance of populations belonging to the Firmicutes, Chloroflexi, and alpha Proteobacteria by at least one order of magnitude. In contrast, these populations decreased in the open circuit and no electron donor amended controls. To better characterize the active microbial populations, we enriched and isolated a novel organism, strain JR, from an operating MFC. Based on 16S rRNA sequence analysis, strain JR has been identified to have a $98 \%$ similarity with a known bacterial species. Phenotypic characterization revealed that strain JR was capable of thermophilic dissimilatory reduction of insoluble electron acceptors such as amorphous Fe(III). Similarly to other known Fe(III) reducing bacteria, strain JR was also capable of producing current by coupling acetate oxidation to anodic electron transfer. This represents the first organism isolated from a thermophilic microbial fuel cell and also the first representative of this genus capable of anodic electron transfer. The results of this study indicate the potential advantages for thermophilic MFCs and the novel microbiology associated with their operation.

B. Research on the cathodic chamber. Our lab has also focused on the cathodic chamber, its potential for biological activity, and the final oxidant utilized. To this end we focused our efforts on stimulating the biological reduction and treatment of perchlorate by perchlorate reducing bacteria (PRB) in the cathodic chamber. In FY06 we demonstrated that perchlorate bioreduction could be stimulated in a cathodic chamber and developed a novel bioreactor based on this. As part of that study we isolated a novel perchlorate reducing organism strain VDY. Over the last year we performed an indepth characterization analysis of this organism and compared its phenotype and genotype to known perchlorate reducing bacteria. 
Micro-characterization and chemical micro-dynamics of atmospheric mineral dust

Principle Investigators: Donald J. DePaolo and John N. Christensen

\begin{abstract}
Project Description
The objective is develop isotopic analysis and modeling techniques to better characterize the sources and transport of atmospheric mineral dust and the chemical and physical transformations that affect its properties. Mineral dust is an important component of the atmospheric aerosol budget. It is now recognized that dust travels long distances in the atmosphere, even to the point that a substantial fraction of the dust present over the western U.S. comes from Asia. Dust therefore constitutes a means of intercontinental material exchange and has important health and security implications. Dust also affects the atmospheric radiation balance and therefore is a critical component of the global climate change. Our objective is to evaluate the extent to which isotopic techniques can be used to fingerprint dust sources and evaluate how these techniques can be best applied. A critical aspect of this is sampling techniques. Another aspect is microchemical analysis and imaging. A third component of the study is the development of Lattice-Boltzmann codes for modeling the micro- physics and chemistry of dust grain surfaces. This project combines lab capabilities in isotopic geochemistry, synchrotronbased analysis and imaging, atmospheric transport, and computational physics and chemistry.
\end{abstract}

\title{
Accomplishments
}

In the third year of the project we have, in collaboration with researchers at U.C. Davis and the California Air Resources Board, we have carried out a study of filters from Heifei, China, taken in Spring 2002 during a dust storm that eventually carried dust to the western U.S. Strontium isotopic measurements, combined with chemical analysis, clearly show that the aerosol load has three sources - local eastern China lowlands, the Western Mongolian-Northwest China deserts, and the marine atmosphere over the adjacent ocean. Tracer results were compared with model air mass trajectories and are in excellent agreement. The isotopic results also allowed portioning of sources and calculation of atmospheric mass loadings of dust aerosols. We have also developed techniques for isotopic analysis of continuous aerosol sampling records. The aerosols are laid down on a strip of mylar, in contrast to a bulk filter sample, allowing for finer temporal resolution. We have been able to analyze such samples taken on Jeju Island, S. Korea that overlap and continue the Hefei time-series. The analyses are consistent with the Hefei samples, and fall on a mixing line between dust and marine aerosol.

To aid in developing models for chemical reactions at dust particle surfaces we adapted a 2D Lattice Boltzmann code for the simulation of microscale kinetics of crystal growth from a fluid medium. The code now handles multiple reacting species, accurately conserves mass, and accommodates the boundary conditions necessary to appropriately simulate surface reactions. We have used it to model the growth of ice crystals in air as a function of temperature and water vapor oversaturation, and the $18 \mathrm{O} / 16 \mathrm{O}$ and $\mathrm{D} / \mathrm{H}$ isotopic fractionation associate with crystal growth at different conditions. The results will allow for improved understanding of isotopic fractionation in the global water cycle. 


\section{The Behavior and Impact of Nanoparticles in the Environment}

Principal Investigator: Benjamin Gilbert

\section{Project Description}

Natural nanoparticles are virtually ubiquitous products of inorganic precipitation and weathering, as well as both sources and products of microbial metabolism. They participate in numerous biogeochemical processes but their impact depends on factors such as redox behavior and transport properties that are generally not well characterized. Research in many areas is required to understand the fate of nanoparticles in the environment, and for beneficial applications of nanoscience in the application and monitoring of remediation technologies. In the final year of the continuing LDRD proposal we concentrated on investigating size effects on the chemical properties of nanoparticles.

\section{Accomplishments}

An important goal of the LDRD is to address the electronic and chemical properties of natural nanoparticles. Previously, we used soft-x-ray absorption and emission spectroscopy at the LBNL ALS to reveal differences between the band-edge electronic states in a variety of ferric iron oxide and oxyhydroxide nanoparticles (hematite, maghemite and goethite). The observation of changes in band-edge electronic structure in nanoparticles versus bulk materials implies that the nanoparticles may more efficiently drive aqueous chemical reactions when illuminated. In 2007 work, we have developed a methodology to connect the results of x-ray spectroscopy with actual observations of aqueous chemical reactions. Specifically, we suspend the nanoparticles in aqueous solution containing a $\mathrm{pH}$-independent redox-sensitive dye (methyl viologen, "MV") and observe the extent of $\mathrm{MV}$ reduction as a function of $\mathrm{pH}$ when the nanoparticles are illuminated. Increasing the $\mathrm{pH}$ raises the absolute energy positions of electrons that are photoexcited to the conduction band, and a threshold in MV reduction is observed when the conduction band energy exceeds the electrochemical potential for MV reduction.

The principal goal of this work was to verify that x-ray spectroscopy can be used to predict the electrochemical potential of photoexcited conduction band electrons. We investigated this for relatively large $(40 \mathrm{~nm})$ hematite nanoparticles, for which the band-edge states were observed to be identical to bulk hematite. We attained very good agreement in the energy position of the hematite conduction band measured by the aqueous photochemical method and $\mathrm{x}$ ray spectroscopy. We anticipate that this study will be important for the development of soft $\mathrm{x}$ ray spectroscopies in characterizing nanomaterials with potential applications in solar energy technologies. More work will be required to ensure that the results are applicable to other systems, and it is hoped that our proposal to the DOE Solar Energy call (see below) will facilitate this research now that the LDRD period has expired.

Our 2006 results were the basis of a full proposal submission to the recent DOE Solar Energy Program. The goal of this proposal is to validate the predictions of the x-ray spectroscopy by demonstrating that the nanoparticles exhibit greater reactivity than the bulk phase for specific aqueous (photo)redox reactions. We are still waiting for DOE to announce the proposals that will receive funding in a second round. The work performed in 2007 will provide a substantial start on the solar energy project if funded. In addition, 2007 research was valuable for a LBNL-UCBerkeley contribution to a preproposal for a major NSF-funded center on the impacts of nanomaterials on the environment (NSF 07-590). 
Compositional and Functional Analysis of Cell-Wall during Metal-Bacterial Actions

Principal Investigators: Hoi-Ying N. Holman, Ping Hu, Sharon Borglin

\section{Project Description}

The purpose of this project is to initiate the development of a dual imaging technology at the Advanced Light Source. It builds upon the novel tunable vacuum ultraviolet (VUV) nanoimaging of biomolecules (developed by the Ahmed Group) at the Chemical Dynamics Beamline and our Fourier transform infrared spectromicroscopy of live cells at the Infrared Beamline. These results will enable us to further the development of existing molecular microprobe tools for characterizing bioremediation of heavy metals. Data generated also would allow us to help creating computational tools to infer both immediate and long-term stability of these biologically transformed metal ions in subsurface environments, which will enable a more realistic assessment of sustainable bioremediation technologies.

We will develop a cell-wall-like system in which its physical and chemical properties can be rigidly controlled for investigating biomolecule-metal interactions. After establishing these model systems, we will use them as test beds for the development of the VUV nano-imaging, and for the extension of the existing infrared micro-imaging technology. We will test the existing instrumentation components and operation parameters, and will modify them if necessary. These data will also be used to extend existing molecular dynamic algorithms, which will enable a more in-depth analysis of the dynamics of an interacting system.

Accomplishments

Our most significant accomplishment has been to advance the reproducible protocol that we developed in FY2006 for preparing cell-like giant unilamellar liposomes (as an outermembrane system) for entrapping macromolecules, and for studying their interactions with metal ions and the long term stability of the complexes.

These cell membrane-like liposome systems are designed with specific attention to meet conditions required by the novel technique of the tunable vacuum ultraviolet (VUV) nanoimaging of biomolecules at the Chemical Dynamics Beamline and by the emerging technique of the Fourier transform infrared (FTIR) spectromicroscopy of live cells at the Infrared Beamline.

To improve the capability of making well-controlled giant liposomes, we also have designed an electroformation cell feasibility study, which will be tested in FY2008.

Dr. Kristina N. Woods, who joined us as a full-time postdoc to work with the PIs on the project, used the interdisciplinary experience she gained from this LDRD project and had successfully obtained a faculty position at the prestigious Carnegie Mellon University. Mr. Schuyler Kain, who joined us as a full-time research assistant to work on the LDRD project, also used the interdisciplinary experience he gained and had become a PhD student in the nanomaterial science group at the University of Wisconsin.

Finally, we have succeeded in obtaining funding support from DOE to purchase a micromanipulator, which allows us in the future to have precise controls on experiment conditions. Based on our cell-wall expertise gained from this LDRD project to date, we have prepared a successful proposal to extend one aspect of our LDRD experience into further frontiers of bioenergy research. 


\section{Interrelation of Global Warming and Hydrate Dissociation in Oceanic Accumulations George Moridis and Matthew Reagan}

\section{Project Description}

Great quantities of methane hydrate exist in deep ocean sediments, where low temperatures and high pressures promote formation and stability. Estimates report total hydrate deposits of up to $10^{19} \mathrm{~m}^{3}$ worldwide. An increase in the temperature of the ocean water in contact with the seafloor could induce dissociation of these hydrates, leading to the large-scale release of methane, a powerful greenhouse gas, into the atmosphere. There is considerable concern that such a release could have dramatic climatic consequences and lead to a disastrous feedback loop. Dissociating hydrates have been suggested as a contributor to rapid and significant climate change in the past, and should be investigated as a contemporary concern.

The purpose of this study is 1) to investigate the effect of rising ocean temperatures on oceanic hydrate accumulations and to determine under which conditions carbon release is likely to occur, 2) to estimate the quantity of carbon that could escape into the environment, accounting for hydrate thermodynamics, fluid transport, and chemistry, 3) to provide a hydrate dissociation model that can be coupled to climate models to determine the overall effect on global climate, and 4) to test the hypothesis that oceanic hydrates may contribute to rapid climate change.

\section{Accomplishments}

We have investigated, through numerical simulation, the effects of ocean warming on the stability of oceanic hydrate deposits using the TOUGH+HYDRATE code. Deep, cold hydrate systems are found to be insensitive all but the most extreme temperature changes, eliminating concerns about the vast deep-ocean hydrate reservoir. For deposits at moderate or shallow depths (known Gulf of Mexico and Arctic Ocean hydrates) we find significant response to temperature changes. Ocean bottom-water temperature increases of as little as $1^{\circ} \mathrm{C}$ over the next century could dissociate these hydrates, releasing methane in both gaseous and aqueous form. Such dissociation would continue until the deposit is depleted. Scenarios with the greatest rate of methane release produce more methane than anaerobic oxidation could be expected to consume, and high fluid velocities may inhibit carbonate sequestration and push considerable gaseous methane through the thin benthic reactive zone into the ocean water column.

For the coldest, shallowest hydrates, such as found on the arctic continental shelf, small temperature increases can produce rapid hydrate dissociation with continuous production of methane gas and high fluid flow rates transporting gaseous and aqueous methane to the seafloor. Less permeable sediments may entrain more of the methane gas and slow transport, and thick layers of overlying hydrate-free sediments may delay the appearance of methane, but any change in temperature shifts the limits of the hydrate stability zone such that the release of gas, even if attenuated, is inevitable.

When applied to the case of a contemporary scenario - shallow hydrates in the Barents Seathe dissociation model indicates that both gradual changes, through warming, or rapid changes, through shifts in ocean currents, could cause rapid release of significant quantities of methane. If this methane does not escape to the atmosphere as gas, it would still threaten the ecosystem of the Arctic Ocean through a severe disruption of ocean chemistry. If it does escape into the atmosphere, it could contribute to arctic ozone depletion as well as to global climate change. Follow-up research coupling these results to future hydrate surveys, global climate models, and atmospheric chemistry is crucial to understand the possible risks. 


\section{A New Technology for Permeability Enhancement for Natural Gas Extraction in Tight Reservoirs}

\section{Principal Investigator(s): Seiji Nakagawa and Andrea Cortis}

\section{Project Description}

With the dwindling supply of foreign and domestic oil and the push to reduce $\mathrm{CO}_{2}$ emissions, natural gas is destined to be one of the premier energy sources by the next decade. A significant part of technically recoverable gas is believed to be trapped within very low-permeability, "tight" gas reservoirs. The primary objective of this project is to develop an entirely new approach for enhancing the production of natural gas in tight reservoirs. The approach employs a self-growing, high-permeability "slot" that is driven from a borehole. The slot is advanced through a coupled hydro-mechanical process in which large stress concentrations around the borehole (or the advanced slot) in concert with high velocity fluid circulation combine to advance a permeable plane through a repeated process of stress concentration $\rightarrow$ rock failure $\rightarrow$ hydraulic debris removal. This method is potentially superior to the conventional hydraulic fracturing method to enhance formation permeability, because the orientation of the slot growth is optimal to encounter preexisting fractures.

\section{Accomplishments}

A laboratory setup was built to apply a low-to-medium-speed water jet (flow speed up to about $100 \mathrm{~m} / \mathrm{s}$ ) to a rock sample during a borehole breakout experiment (a specially designed setup was developed during the previous year). The purpose of the water jet is to remove debris from a borehole surface and advance a slot-shaped breakout. For a very tight and strong synthetic rock core (porosity 7\%), the jet successfully created a narrow, slot-like breakout. With the current power of the jet, however, we found it difficult to advance the slot further deep into the rock. This may be partly due to a too high strength of the synthetic rock (estimated uniaxial compression strength $>200 \mathrm{MPa}$ ). Also, this result may indicate that an application of this technology in the field requires an insertion of a micro-tubing into the slot to sustain the jet strength.

For a better understanding of the physical processes that govern the evolution of the debris erosion, we have implemented a quasi-stationary, dynamically coupled computer code. This code computes the water-jet induced stress on a granular rock matrix, and compares it against the intergranular cohesive force that holds the granular aggregate together. The minimum energy configurations of the sandy aggregate are calculated by means of a Discrete Element Model (DEM) developed in collaboration with Dr. Bruno Chareyere (Institut National Polytechnique de Grenoble, France). The turbulent jet fluid flow simulations are performed by means of the commercial Finite Element Method (FEM) code COMSOL-Multiphysics. The interface between the DEM and the FEM modules was developed in the Matlab language. The hydraulic coupling between the two codes has been so far tested on a small number of spheres, and its numerical performance needs to be assessed for more realistic sandy aggregates. The full numerical solution of this problem may require the use of large computing facilities. To our knowledge, this is the only existing working example of a fully coupled hydro-mechanical code. 
Coupled Modeling of Hydrology, Nutrient Cycling, and Vegetation: Applications to Water Quality and Water Balance

Principal Investigators: Curt Oldenburg, Bill Riley, Norm Miller (LBNL) Post-doctoral fellows: Federico Maggi, Chuanhui Gu (BWC)

Project Description

This joint Earth Sciences Division (ESD) and Berkeley Water Center (BWC) LDRD project is focused on understanding biogeochemical cycles of nitrogen species and production of $\mathrm{NO}, \mathrm{N}_{2} \mathrm{O}$, and $\mathrm{CO}_{2}$ gas and $\mathrm{NO}_{2}{ }^{-}$and $\mathrm{NO}_{3}{ }^{-}$solutes in nitrogen-rich agricultural fields, and the interaction between soil microbial biomass, the hydrological cycle, plant dynamics, and climate forcing. The motivation for the effort is to understand the release of $\mathrm{NO}, \mathrm{N}_{2} \mathrm{O}$, and $\mathrm{CO}_{2}$ from the soil surface to the atmosphere as a key factor in controlling the greenhouse effect, a topic assuming ever greater importance in light of expected increases in crop-based biofuels, food, and fiber production. The objectives of this research are to develop a mechanistic model (TOUGHREACT-N) for N cycling and losses from agricultural fields, and to use this model to investigate effects of fertilizer application amount and type, water application, and fertilizer application depth.

We have developed the model TOUGHREACT-N, based on TOUGH2 and TOUGHREACT, for various nitrification and denitrification pathways, multiple microbial biomass dynamics, hydrological water flows, and various chemical reactions under local equilibrium and kinetic controls. The soil column is represented as a 1D vertical column with hydraulic properties described by a water tension/saturation model. Biotic and abiotic reactions are assumed to follow Michaelis-Menten kinetics, while biomass is assumed to follow multiple Monod growth kinetics accounting for electron donor, electron acceptor, and inhibitor concentrations. Water flow is modeled with the Darcy-Richards equation, while nutrient transport is modeled by Fickian advective and diffusive processes in both gaseous and liquid phases.

\section{Accomplishments}

We have tested TOUGHREACT-N against $\mathrm{pH}, \mathrm{NH}_{4}{ }^{+}, \mathrm{NO}_{2-}$, and $\mathrm{NO}_{3}{ }^{-}$ion concentrations, and $\mathrm{NO}, \mathrm{N}_{2} \mathrm{O}$, and $\mathrm{CO}_{2}$ gas emission data from an experimental site in Sacramento, CA. Good agreement between field observations and model predictions has been found for all quantities, and a publication on this work is in press in JGRBiosciences (Maggi et al, 2007). We have also carried out a comprehensive analysis of gaseous emissions and solute leaching from various fertilizers with good agreement with measurements collected in a field site in Burgundy, France, and have manuscript is under consideration in JGR-Bio ( $\mathrm{Gu}$ et al 2007). We are in the process of coupling TOUGHREACT-N with a land-surface model. 


\section{Biogeochemical Reaction Rates and Pathways in Porous Media Principal Investigator: Carl Steefel}

\section{Project Description}

The purpose of this project is to dramatically increase our understanding of the scale dependence of biogeochemical and geochemical reaction kinetics in natural porous media. The present lack of understanding limits our ability to develop effective bioremediation schemes for contamination cleanup, to develop predictive models for $\mathrm{CO}_{2}$ sequestration in deep aquifers, and even to determine the fundamental controls on the rates of chemical weathering, an important long-term regulator of atmospheric $\mathrm{CO}_{2}$ levels.

\section{Accomplishments}

Reductive Dissolution of Fe-Hydroxides: During the reduction of Fe-hydroxides by dissimilatory iron-reducing bacteria, the ferrous ion reaction products will absorb on to the surface of remaining Fe-hydroxides surfaces and result in the structural transformations of Fehydroxides by electron transfer processes. The detailed mechanisms and pathways of these $\mathrm{Fe}(\mathrm{II})$-catalyzed transformations of Fe-hydroxides are still largely unknown. Synthetic ferrhydrite was selected as the starting material because of its large capacity as scavengers for environmental contaminants. Inorganic ferrous ion solution was directly injected into $100 \mu \mathrm{m}$ I.D. capillary tubes packed with ferrihydrite-coated glass spheres to study its transformation pathways and rates during flow through the micro-porous environment. The phase transformations were monitored by synchrotron XRD method on ALS beamline 11.3.1 and 10.3.2. The reaction products were further examined by Transmission Electron Microscopy at NCEM of Berkeley lab. The study results showed a complete conversion into magnetite without any intermediate phase being detected. The transformation rates are very rapid compared to the values obtained in traditional bench reactors. In addition, instead of a constant rate, the transformation rates were slow at the beginning, and then increased dramatically with time until most of the ferrihydrite was consumed. The overall fast transformation process indicates a solid state transformation mechanism and the rates profile showed a dispersive kinetic behavior. Continuing experiments with another important Fe-hydroxides phase, goethite, are currently under preparation.

Kaolinite Precipitation Kinetics: Experiments on the precipitation kinetics of kaolinite showed a complex behavior. One conducted using kaolinite seed that had previously undergone extensive dissolution under far from equilibrium conditions for 5 months showed a quasi-steady state precipitation rate for 105 hours that was compatible with the TST expression above. After this initial period, however, precipitation rates decreased by an order of magnitude, and like other precipitation experiments conducted at higher supersaturation and without kaolinite seed subjected to extensive prior dissolution, could not be described with the TST law. The initial quasi-steady state rate is interpreted as growth on activated sites created by the dissolution process, but this reversible growth mechanism could not be maintained once these sites were filled. Long-term precipitation rates showed a linear dependence on solution saturation state that is generally consistent with a two dimensional nucleation growth mechanism.

Further analysis using Synchrotron Scanning Transmission X-ray Microscopy (STXM) in Total Electron Yield (TEY) mode of the material from the precipitation experiments showed spectra for newly precipitated material compatible with kaolinite. 


\section{Engineering Division}

Novel Imaging Detectors for Materials and Biology Research Principal Investigator(s): P. Denes and H. A. Padmore

\section{Project Description}

The purpose of this project is lay the groundwork for a new program in imaging detectors. LBNL's long experience in specialized detectors for particle physics and astronomy provide the technical foundation, and this project includes specific R\&D to realize high-impact, new types of detectors. We are focusing on improving the ubiquitous detector in synchrotron radiation research and electron microscopy, based on a scintillating phosphor fiber-coupled to a CCD. In order to improve sensitivity and live time, as well as enabling new kinds of dynamic experiments, we have developed a highspeed CCD which can be read out at the rate of $5 \mathrm{~ms}$ per megapixel while maintaining a dynamic range in excess of 14 bits. This combination of speed, dynamic range and number of imaging pixels is unprecedented. In addition, we are developing an innovative electron microscopy detector, which will not only be fast and sensitive, but provide excellent spatial resolution - particularly at the higher energies which are of interest for thick biological specimens.

\section{Accomplishments}

During FY07, we fabricated and tested the 16-channel custom integrated circuit used to readout the fast CCD described above. Based on the concept developed for the SNAP experiment, the fast CCD IC runs ten times as fast, and packs four times the number of channels into the same space as the SNAP chip. Each channel includes a preamplifier, floating point gated integrator, correlated double sampler and pipelined ADC. The circuit was fabricated in $0.25 \mu \mathrm{m} \mathrm{CMOS} \mathrm{technology,} \mathrm{and} \mathrm{performs} \mathrm{as}$ expected.

During FY07, we also developed an all-silicon hybrid technology to mount the CCD and custom readout chips on a silicon substrate. This allows good thermal expansion coefficient matching, so that the entire assembly can be cooled. While similar to a multi-chip module, the all-silicon hybrid uses a thick silicon substrate.

We also continued design refinements for a fast electron microscopy detector, implementing a pixel with in-pixel correlated double sampling. Dark field images confirm a sensitivity of about 1000 times that of film. 
Understanding the chemistry of innovative air cleaning technologies

Principal Investigator: Hugo Destaillats

\begin{abstract}
Project Description
The goal of this project is to investigate the use of ozonation, photocatalytic oxidation, sorption and chemisorption treatments for the removal of volatile organic compounds (VOCs) from indoor air. Air-cleaning technologies employing these combined approaches can achieve important pollutant removal enhancements while extending the lifetime of catalysts and sorbents. Effective air cleaning devices may contribute significantly to improving the energy efficiency of ventilation systems. We intend to describe the underlying chemical basis by developing a technique for the characterization of gas-phase and sorbed reaction products and intermediate species generated in the reaction of model pollutants. This analysis will allow us to characterize the performance of innovative sorbents and catalysts designed using novel meso- and nanoscopically tailored materials.
\end{abstract}

\title{
Accomplishments
}

Several research efforts are currently underway. We designed and built an Attenuated Total Reflectance (ATR) flow cell reactor which allows for the simultaneous examination of surface and gas-phase reactants and products formed during the irradiation of a $\mathrm{TiO}_{2}$ thin film with UV light in the presence and absence of ozone. This system was initially used to characterize carboxylic acids adsorbed on standard $\mathrm{TiO}_{2}$ samples (Degussa P25). We also evaluated the formation of surface-bound species during photooxidation of other organic compounds, such as alkylnaphthalenes and cyclic syloxanes. Oxidation of these chemicals is considered to be involved in inactivation mechanisms that affect the long-term performance of $\mathrm{TiO}_{2}$-based photocatalytic oxidation (UVPCO) air cleaners. We identified stable oxidation products that are likely responsible for site inactivation, and verified their inertness under UV irradiation. Once the chemical mechanisms were elucidated, we used the same experimental approach to test novel materials being developed as alternative photocatalysts, in order to quantitatively determine their enhanced performance with respect to inactivation.

The ATR flow system was also used to evaluate the performance of novel $\mathrm{TiO}_{2}$ clay nanocomposites synthesized and characterized specifically for this project. These materials showed a photocatalytic activity that was comparable with that of P25, and in one case (hectorite composites) a significantly larger active surface area for VOC adsorption. A different flow reactor was used for the performance evaluation of photocatalytic oxidation of toluene (a model VOC) under various experimental conditions, including UV frequency of irradiation, relative humidity and catalyst film thickness. 
Exploring Electrochemical Arsenic Remediation of Drinking Water for Bangladesh Principle Investigators: Ashok Gadgil, Robert Kostecki, Venkat Srinivasan

\section{Project Description}

This project aims to investigate accelerated formation of $\mathrm{Fe}(\mathrm{OH})_{3}$ and $\mathrm{FeOOH}$ for affordably removing arsenic from drinking water in Bangladesh, where 60 million people drink arsenicladen water from shallow wells. Approaches currently considered suitable for Bangladesh use rusted metallic iron (usually nails) as a source of ferric hydroxide $\left(\mathrm{Fe}(\mathrm{OH})_{3}\right)$ or ferric oxyhydride $(\mathrm{FeOOH})$ which bind the arsenic into insoluble ferric arsenate. This approach is limited by the natural rate of $\mathrm{FeOOH}$ production given an affordable quantity of nails. We intend to increase $\mathrm{Fe}(\mathrm{OH})_{3}$ or $\mathrm{FeOOH}$ production rate from metallic iron by factors of 10 to 100 using electrochemistry, substantially reducing the first-cost barrier to using this technology, and investigate the performance of this approach in synthetic and real Bangladesh water samples.

We will develop a small-scale device that will be analyzed with electrochemical system models to permit performance prediction of full-scale devices. In addition, we will study the chemical reactions of interest in a synthetic ground water with the same composition, $\mathrm{pH}$, dissolved oxygen, and arsenic content of typical Bangladeshi ground water. This will allow us to understand how the reactions will behave in the unique Bangladeshi water environment, an area of study that has so far been neglected in the literature. The questions we will answer are related to the effects on the electrochemical system of the specific ionic species in the raw water, the $\mathrm{pH}$, dissolved oxygen and temperature effects on system performance, and the engineering design to make the system robust, fail safe, and most importantly, affordable to rural poor Bangladeshis.

\section{Accomplishments}

First project year demonstrated a working electrochemical device and measurements of the arsenic removal capacity in synthetic Bangladeshi groundwater over a small range of charge and current densities. Important achievements in the current year include successfully reducing the arsenic in two Bangladesh water samples to below the WHO health limit of $10 \mathrm{ppb}$ using our device. We designed and built a second electrochemical cell as a small prototype batch reactor and tested it over a small range of charge and current densities using a second recipe for synthetic Bangladeshi groundwater. This second recipe is designed to mimic the "worst case scenario" water in Bangladesh for arsenic removal, including the highest ion concentrations measured in Bangladeshi water for several key ions known to interfere with arsenic removal. We have also designed and built a hand-held household scale prototype and measured its arsenic removal capabilities across current and charge densities.

In addition we have performed a number of experiments to uncover the mechanism of arsenic removal in our process. Cyclic voltammetry and Impedance Spectroscopy using iron electrodes in synthetic Bangladeshi water have been performed, revealing the probable chemical reactions occurring in different regions of current density. EXAFS and XANES spectra at the Arsenic and Iron K-edges have been measured at the ALS and SSRL to identify the key iron hydroxides or oxyhydroxides responsible for removal. Optimization of the electrochemical removal process thus far has reduced the estimated operating costs to 1 US cent per person per day, assuming 10 Liters per person and a photovoltaic electricity source. An invention disclosure has been filed and is expected to lead to a patent filing by UC/LBNL. 
Soot in Ice: Does Soot Enhance the Melting of Ice?

Principal Investigator: Thomas Kirchstetter

\section{Project Description}

This project aims to examine the effect of black carbonaceous soot contamination on the melting of snow and ice. General circulation and radiation models used in the prediction of climate indicate that the perturbation by soot in snow and ice, which reduces the surface albedo (the fraction of incident sunlight that is scattered back to space), may be responsible for a significant portion of the global warming of this past century, including thinning of Arctic sea ice and melting land ice. A number of studies provide estimates of the amount of soot in surface snow/ice and precipitation, but there is little experimental or empirical data that support theoretical estimates of the radiative effects of soot in snow/ice. This project aims to provide data - measured changes in surface albedo and in the rates of heating and melting of snow/ice/soot systems exposed to light - to complement field measurements by others and to corroborate or refute theoretical estimates.

The project involves 1 ) developing methods of making standardized ice and snow samples contaminated with known amounts of well-characterized soot and 2) conducting experiments that measure the effect of soot contamination on the spectral albedo and the heating and melting rates of snow and ice.

\section{Accomplishments}

One significant accomplishment has been the development of a method of making stable suspensions of soot in water. Soot is produced with an inverted, co-flow diffusion flame of methane and air. The soot particles, which are almost entirely elemental carbon in composition, are initially hydrophobic. Through oxidation of the particle surface with ozone, the soot is transformed from hydrophobic to hydrophilic, at which point it readily forms homogeneous suspensions in water. A specialized, highly sensitive, optical spectrometer provides detection of soot in water at the low levels found in environmental samples - as low as 1 nanogram of soot per gram of water. Through these measurements, the mass absorptivity of soot in water has been determined over a broad spectral region. This work demonstrates that it is possible to use optical spectroscopy to quantify soot in melted samples of snow and ice, a technique that is much simpler and likely much less error prone than other techniques currently employed by other investigators.

Temperature-controlled chambers have been used to make and observe pristine and sootcontaminated ice samples. Preliminary results indicate that soot-contaminated ice samples heat at a faster rate than pristine samples when exposed to light. The project will continue with measurements to improve our confidence in these initial results. Our observations demonstrate the redistribution of soot when a homogeneous suspension of soot in water freezes: the soot becomes concentrated in the water that freezes last. This redistribution may have a significant impact on the evolution of the radiative forcing of soot in snow and ice during periods of melting and refreezing. 
Surface Plasmon-Enhanced Photovoltaic Device

Principal Investigators: Robert Kostecki, Samuel Mao

\section{Project Description}

The purpose of this project is to develop and test an entirely different approach to produce a prototype of a photovoltaic (PV) device. This new concept offers an opportunity to create a new class of photovoltaic devices as compared to presently known PV systems. We investigated whether intense photoemission of hot electrons from nano-structured thin-films can be used to design and develop a new class of plasmonic PV devices. We proposed that photo-excited plasmons in a thin film nano-structured electrode discharge into hot electrons, which can be collected through a semiconductor Schottky diode during the steady illumination of the electrode with near UV and visible radiation. The electrons injected into the diode were detected and measured as a short-circuit current.

The project goals are to: (i) detect and quantify the plasmon-assisted electron emission at a nano- patterned electrode/Schottky junctions, (ii) determine the effect of roughness of the electrode surface on the spectral range of optical excitation of SP's and the magnitude of photocurrent response, and (iii) build a prototype photovoltaic device which operates based on plasmon-enhanced photo-emission. We also carried out studies of dynamic plasmon-assisted photoemission and use the results to develop deeper understanding of charge transfer mechanisms at or near the solid-solid interface. We used ultrafast Pulsed Laser Deposition (PLD) and other vacuum deposition methods to fabricate the photosensistive electrode structure.

Accomplishments

Our primary accomplishment in the past year has been the further advancements in a reproducible experimental protocol for preparing thin-film photovoltaic devices, fabrication of prototype devices via different deposition techniques, and testing. Our methodology combines a standard sputtering, chemical vapor deposition (CVD) and PLD techniques. This experimental procedure involves a p-type $\mathrm{Si}(100)$ and deposit various layers and a nanostructured "sandwichlike" thin-film architecture. The deposition process was optimized to produce thin-films with a high concentration of oxygen vacancies and imperfections to establish the proper energy barrier for the electrode/Shottky diode. It was critical to assure an ohmic contact between the thin-film layer and the electrode. The morphology of the layer was tailored to particle size.

We have succeeded in manufacturing several enhanced prototypes of the PV device. Many of the prototypes displayed a weak (a few nA) photocurrent in response to illumination. The current signal always decayed very quickly and almost completely disappeared after about 60 min. These preliminary results verify positively the concept but also indicate that the diode design and materials must be further optimized to improve the photon collection and charge separation efficiency. 


\section{China’s Energy Future: Changes in Energy Intensity \\ Principal Investigator: Mark Levine}

\section{Project Description}

The topic of China's energy system is receiving increasing attention around the world. In many quarters, this attention has turned to alarm. Coal consumption is growing at an enormous rate (75\% growth in 5 years), power plants are being constructed at the fastest pace in history, and greenhouse gas emissions are growing apace. One-third to one-half of the world's incremental demand for oil has come from China in the past five years. After a long and successful experience in implementing energy efficiency (19802000), China has seemingly lost its ability to carry out energy efficiency to scale. This project has investigated the factors in the Chinese economy that have brought about these changes, in the effort to design ways out of the dilemma.

\section{Accomplishments}

The work will produce two separate outputs. The first is an analysis of the factors that have led to the enormous and unexpected growth in energy demand in China from 2001 to the present. The second is a comparison of different energy demand and $\mathrm{CO}_{2}$ scenarios for China.

In the first paper, we have performed statistical analyses to assess the contribution to energy intensity of changes in structure of the economy as a whole. The analysis showed that the increase in energy demand could be largely attributed to increasing energy intensity of industry. At first glance, these results are counter-intuitive. In a rapidly expanding economy, new and more efficient technologies are typically deployed throughout the economy, which should lead to a reduction in energy intensity in industries. However, industrial energy intensity is determined by two factors: 1) energy efficiency in industrial sub-sectors, 2) the relative outputs of the sub-sectors. Thus, it is possible that overall industrial energy intensity could increase, even when energy efficiencies at the sub-sector level are improving because the relative outputs of energy intensive sub-sectors such as cement and iron and steel are rising. This is in fact what has happened in China since 2001.

A second element of this work involved analyzing what would be necessary for China to achieve its goal of reducing energy intensity by $20 \%$ in five years. The conclusion of the analysis was that China could achieve about half of the desired goal by energy efficiency, but that success would depend on China's ability to reduce the growth of energy-intensive industry and to close very inefficient facilities.

Work on a review paper describing energy and $\mathrm{CO}_{2}$ scenarios for China has demonstrated the failure of the most widely used models to predict the rapid growth of energy demand in China since 2001. As late as 2004, the major model results did not have China overtaking the United States in energy-related $\mathrm{CO}_{2}$ emissions until 2020, when that happened in 2006 or 2007. The paper will explore what can be learned from the models' failure to improve modeling of energy demand. 


\section{Integrated Decision Support Tool for Joint Optimal Control of Energy and Water Systems under Uncertainty}

James McMahon, Larry Dale, Juan Meza, Peter Schwartz, Boris Faybishenko, Norman Miller, David Sunding

\section{Project Description}

In this project we investigate water-energy trade-offs and the impact of changes in climate, electricity prices and water supply on resource management. We will focus on the water supply process from reservoir storage to conjunctive groundwater storage, irrigation, and delivery, and provide a systematic estimate of the value of water and energy conservation. We will also evaluate net energy requirements with water use options, including water for ethanol production. We will develop robust decision support tools using dynamic programming for optimal control of energy and water systems. We will develop an integrated decision support tool from existing energy and water models, use explicit characterizations of sources of uncertainties, and develop a ranking of key uncertainties as targets for further research. Finally, we will develop a clear path forward for applying this technology.

We hypothesize that optimal control of our proposed integrated system provides a better match between supply and demand for water and energy, fewer failures to meet demand and lower total costs to society. Our approach to these problems brings algorithms from high performance computing to bear on these problems. In particular, we have constructed parallizable adaptive algorithms based on finite difference methods to numerically solve the partial differential equations of stochastic dynamic programming. The most challenging part of this algorithm is developing a second order method for solving the eikonal equation. For high dimensional problems approximate solutions to the PDE will be calculated from neurodynamic algorithms. The results of this work will be summarized into feedback control release rules, to be integrated into the Sacramento hydrology crop model. Our integrated decision support tool will improve our ability to plan given large uncertainties.

\section{Accomplishments}

The LDRD includes four major accomplishments, including definitional, mathematical, modeling and economic accomplishments. We've defined two applications of this work including optimal conjunctive use of surface and ground water and the option-value of anticipated learning about climate-change in the Sacramento Valley of California. We have a working hydrology model of the Sacramento Valley, which indicates river flows, reservoir storage, groundwater pumping, crop water demand and urban water demands in the region. The model is being programmed to allow for changes in water storage, cropping and landscape demands, in response to changes in energy and water prices resulting from climate change. The mathematical group brings high-performance computing algorithms to bear on these optimal control problems in water/energy resource management. The computing algorithms will provide rules for storing water, given changes in water energy and crop prices. We have defined an economic methodology for estimating crop production functions, which show changes in cropping patterns in response to changes in energy and water prices and changes in the price of biofuels. The functions will be programmed into the hydrology Sacramento hydrology model and used to evaluate a variety of policy, economic and climate scenarios. 


\section{Project Title: Bio-oil Accumulation In Unicellular Green Algae - A Pilot Project}

\section{Principal Investigators: Tasios Melis and Rolf Mehlhorn}

\section{Project Description:}

A variety of green microalgae naturally accumulate a large fraction of their biomass as oils, which can be products of either the fatty acid or terpenoid biosynthetic pathways. This project seeks an understanding of the molecular and cellular events that govern the oil-producing processes, which may make it possible to generate large amounts of oils from mass-cultured photosynthetic microorganisms. The oils and related compounds could become important feedstock in the synthetic chemistry industry and serve as renewable biofuels. Elucidation of the associated metabolic processes will improve yields of bio-oils from microalgae and, possibly, from terrestrial plants. The research will exploit the modern tools of genetics, molecular and cell biology. Concurrently, separation of bio-oils from the biomass will be pursued with novel engineering technologies.

This pilot project began by applying DNA insertional mutagenesis, UV-induced mutagenesis and chemical mutagenesis, and further, by applying a stringent high throughput screening protocol for the isolation of mutant Chlamydomonas reinhardtii strains with unusual bio-oil accumulation characteristics. The primary aim was to reach a genome-saturated mutagenesis and high throughput screening, resulting in the isolation of bio-oil accumulating strains of the model green alga $C$. reinhardtii. Successful isolation of such mutants would lead to the cloning of genes and characterization of bio-oil accumulation pathways. The mutagenesis and cloning expertise available in the Melis lab made it possible to expeditiously pursue the objectives of this novel project. As the project evolved, necessary improvements were made in culturing cells, analyzing their composition and separating biomass fractions.

\section{Accomplishments}

Preliminary attempts to isolate mutants with greatly increased oil production by inducing random mutations in $C$. reinhardtii were unsuccessful, owing in part to the limited sensitivity of the assay we used to estimate oil production. We tentatively conclude that the desired mutations in this microorganism are rare, and their detection will require improved assays of oil accumulation within cells.

We shifted our emphasis to the organism that has the desired oil-producing capacity but not productivity, Botryococcus braunii, with the intention of exploring ways by which to increase its rate of growth and oil production. A major challenge in quantifying productivity parameters for this organism is the composition of the biomass, comprising large aggregates of cells that vary in size and density. A significant achievement during the project period was the development of reliable assays of growth rates and hydrocarbon synthesis suitable for working with small sample volumes. Another achievement was the development of GC-MS analytical tools for the rapid identification and quantification of specific oil fractions. Towards the end of the project period substantial progress was achieved in efficiently separating the bio-oils from the biomass while retaining cell viability. 


\section{The Electrolysis of $\mathrm{CO}_{2}$ to a Liquid Fuel \\ Principal Investigators: John Newman and Douglas Clark}

\section{Project Description}

The reasons to move away from fossil-based fuels are prevalent, but the ability of our society to rid itself completely of liquid fuels, especially in the sector of long-haul transportation such as trucks, aircraft, and ships, is not an easily foreseeable option. Thus, to keep from increasing our carbon dioxide emissions while maintaining our present standard of living, it is vital that we discover a means of making liquid fuels from renewable sources in a cost-effective manner. The goal of this project is to evaluate the feasibility of reducing carbon dioxide $\left(\mathrm{CO}_{2}\right)$ to a liquid fuel precursor at a high rate (approaching $0.1 \mathrm{~A} / \mathrm{cm}^{2}$ ) and high energy efficiency $(>50 \%)$. To this end, two electrochemical reactors were investigated: 1$)$ the direct reduction and oxidation of $\mathrm{CO}_{2}$ and water $\left(\mathrm{H}_{2} \mathrm{O}\right)$, respectively, to syngas (hydrogen and carbon monoxide) and oxygen in a high throughput cell utilizing metal catalysts, and 2) the reduction of $\mathrm{CO}_{2}$ to formate (a precursor to methanol) utilizing a redox mediator in the presence of an enzyme. The goal of the project, ultimately, is to demonstrate the ability to make a liquid fuel from $\mathrm{CO}_{2}$ in a single reactor and evaluate the costs versus a more conventional, multiple-reactor scheme.

In the first approach, a flow-by electrochemical reactor was designed with an emphasis on high rate and moderate efficiency $(>50 \%)$. This reactor originally resembled the typical proton-exchange-membrane fuel cell with composite electrode assemblies and a thin proton conducting separator. A number of metallic catalysts, reported to demonstrate preferential reduction of $\mathrm{CO}_{2}$ to $\mathrm{CO}$, were evaluated along with different electrolyte configurations. In the second approach, an aerobic/anaerobic electrochemical reactor was designed with an emphasis on efficiency and selectivity. In this initial investigation, C13-NMR was used to measure the turnover rate of $\mathrm{CO}_{2}$ reduction to formate by intact microbial cells containing the enzyme formate dehydrogenase (FDH) in the presence of reduced methyl viologen.

\section{Accomplishments}

First approach: We devised a novel cell configuration that includes a proton-exchangemembrane (PEM) pressed between the two composite gas diffusion electrodes that results in a measurable amount of $\mathrm{CO}_{2}$ reduction from a short list of candidate catalysts. The selection of a certain metal-based catalyst resulted in high selectivity of $\mathrm{CO}_{2}$ to $\mathrm{CO}$ at a current density of 30 $\mathrm{mA} / \mathrm{cm}^{2}$ at -1.7 to $-1.75 \mathrm{~V}$ versus a SCE (saturated calomel electrode.) At lower potentials, both $\mathrm{CO}$ and $\mathrm{H}_{2}$ are produced. A CO/ $\mathrm{H}_{2}$ ratio of $1 / 2$, suitable for methanol synthesis, is achieved at a potential of ca. $-2 \mathrm{~V}$ versus a SCE and a total current density of $80 \mathrm{~mA} / \mathrm{cm}^{2}$.

Second approach: Enzyme-mediated reduction of $\mathrm{CO}_{2}$ to formate was quantified and directly confirmed in intact cells of Syntrophobacter fumaroxidans by C13-NMR. Measured initial rates indicate a turnover of $100 \mathrm{~s}^{-1}$, which is two orders of magnitude higher than the highly active $\mathrm{CO}_{2}$-reducing FDH from Pseudomonas oxalaticus. Product analysis by $\mathrm{C}-13 \mathrm{NMR}$ did not show an increase of formate yield relative to that seen in the absence of the electrochemical system. We interpret our results to indicate that methyl viologen is unlikely to act as an electron shuttle that diffuses back and forth across the membranes of S. fumaroxidans. 


\title{
Switchable Mirror Prototype Fabrication
}

\author{
Principal Investigators: Thomas J. Richardson and André Anders
}

\begin{abstract}
Project Description
Two different switchable mirror systems were discovered in an exploratory research program supported by DOE Building Technologies. One of these (metal hydrides) was chosen for further development within the ongoing buildings technology program, while the exploratory work and development of devices using the other system (lithium alloys) has not received further funding. LBNL holds patents on both technologies and they have generated considerable interest from a wide variety of industrial firms from window glazing and automobile manufacturers to the aerospace and electronics sectors and solar energy developers. The project called for fabricating selfcontained prototype devices that could be used for demonstration purposes including future funding opportunities. Such fabrication also strengthens our capabilities to proceed from ideas and concepts to actual devices. We proposed to produce the necessary components for assembly of about twenty electrochromic lithium alloy switchable mirror devices. We proposed to use and modify existing coating equipment and prepare switchable alloys and counter-electrodes for testing and further assembly with electrolyte to working prototype devices.
\end{abstract}

\section{Accomplishments}

A co-sputtering system for deposition of tantalum contacts and copperantimony alloy electrodes was set up. The $\mathrm{Cu}$ and $\mathrm{Sb}$ ratio was carefully calibrated and the alloy films produced and optimized such that the thickness gradient was minimal. Small residual visible gradients of optical transmittance could be eliminated by substrate rotation during deposition but that improvement needs to wait for further funding. The thickness of the CuSb films was selected such that the transmittance in the as-deposited (reflecting) state was about $1 \%$ or less. The CuSb films were found adequate and more than 20 films on carefully cleaned glass substrates of about $30 \mathrm{~cm}^{2}$ have been deposited.

In the next step, the counter electrode was produced by co-deposition of titanium oxide and vanadium oxide on indium-tin oxide (precoated) substrates of the same size. The counterelectrode is transparent with a slight yellow tint. The counterelectrode capacity for lithium was measured in an electrochemical cell prior to assembly of the switchable mirror device using a lithium-containing polymer electrode. The devices were sealed with epoxy so that they could be removed from the glove box and operated with a low-voltage power supply or batteries.

A local solar energy company has now purchased an option to license this technology from Berkeley Lab. Discussions are also in progress to establish a Work for Others project with this firm. 


\section{Genomics Division}

Computational and Experimental Testing of Methods for Binning Sequences from Metagenomic Studies

Principle Investigator: Jonathan A. Eisen

\section{Project description}

The purpose of this project is to improve upon our ability to perform metagenomic analysis (sequencing DNA directly from environmental samples). We have two main goals. First is the development model metagenomic data sets where the organism source of the DNA is known. This will allow the testing of bioinformatics methods that attempt to assign sequence reads to organisms (generally known as binning) which is a critical step in metagenomic analysis. Our second goal is to develop new methods for performing binning, making use of the model data sets to test our methods against other methods that have been described.

\section{Accomplishments}

We are taking two approaches for the development of model metagenomic data set computational simulations and laboratory simulations. For the computational simulations we have generating simulated metagenomic data sets by (1) selecting sets of completed genomes and (2) randomly sampling the original sequence reads from those genome projects where the number of sequence reads from each organism is varied to create different ratios of mixes of organisms. We have generated twenty simulated data sets and are using them to test various binning algorithms since we know the "answer" regarding which reads map to which organisms.

For our laboratory simulations, which are just beginning, we have selected ten organisms which are easy to grow and which have their complete genomes determined. We have grown these species, counted cells in cultures, and made pools that are predicted to contain the same number of cells. We then extracted DNA from these pools and used this DNA for metagenomic sequencing using both Sanger and 454-Roche sequenc ing methods. We are in the process of analyzing these data.

One paper making use of some of the computational simulations has been submitted. 


\title{
Metagenomics-Enabled Analysis of Termite Hindgut Microbiota for Biomass Conversion and Cleaner Energy
}

\author{
Principle Investigator: Philip Hugenholtz
}

\section{Project description}

Termites efficiently transform recalcitrant plant biomass, lignocellulose, to valuable sugars and fuels (hydrogen, methane) by exploiting the metabolic capabilities of microbial symbionts inhabiting their hindguts. An understanding of the enzymes and biochemical pathways used in the termite hindgut will therefore lead to more efficient strategies for converting biomass to fuels and chemicals. However, the mechanisms used to achieve these transformations remain elusive in the absence of molecular data. The purpose of this proposal was to begin the research to reconstruct the metabolic pathways responsible for biomass conversion in termites using a metagenomic approach, paving the way for industrial applications such as biological ethanol and hydrogen production.

\section{Accomplishments}

During FY07 the analysis of the metagenomic sequence dataset obtained from lumen fluid from the P3 hindgut segment of the higher termite Nasutitermes was concluded. A manuscript concerning the analysis has been prepared and published in Nature. Based on this research LBNL is in negotiations with a company concerning licensing on P3 cellulases. In addition a material transfer agreement (MTA) has been signed so that the alkaline P1 segment samples collected from Nasutitermes workers in May 2006 were sent to JGI for metagenomic sequencing and analysis.

LBNL has decided to focus on $\mathrm{C} 4$ grass feedstocks for its cellulosic ethanol program. Therefore, grass-feeding higher termites belonging to the genus Gnathamitermes may be more relevant than the drywood-feeding Nasutitermes, and would also provide a useful comparative system for understanding lignocellulose hydrolysis. Gnathamitermes are found within the US which aides in sample acquisition. To our knowledge, no research has been conducted on Gnathamitermes hindgut microbiota. Therefore, we conducted a sampling trip to southern Texas with our collaborator Dr. Rudolf Scheffrahn (U Florida) who is an entomologist specializing in termites. We collected specimens of higher termites from 4 different species (Gnathamitermes tubiformans, Amithermes wheeleri, Anoplotermes fumosus, Tenuirostritermes cinereus) at different locations. We plan to assess P3 and P1 community structure and function by $16 \mathrm{~S}$ PCR clone libraries and metagenomic sequencing. Due to the end of the LDRD funding period, we will pursue this research using a grant from the Energy Biosciences Institute (EBI) which was accepted for funding at the end of 2007. 
Project Title: Development of Cost Effective Sequence-Based Technologies to Identify Genomic Alterations in Cancer

Principal Investigators: Len Pennacchio, Paul Spellman, Jan-Fang Cheng

\section{Project Description}

Recurrent somatic chromosomal aberrations in malignant cells have been identified as a principle cause of cancer development and progression. The technology capable of detecting these aberrations has been slow and costly and as a result the vast majority of such events remain unidentified. The purpose of this project has been to develop a cost-effective, high-throughput sequence-based approach to drastically increase our ability to identify structural alterations in genomic DNA. The resulted technologies will enable systematic efforts to catalog all structural variations in the non-cancerous and cancerous genomes and a robust detection of genes disrupted by these aberrations.

We have been developing and improving two technologies. The first technology (aka DiTags) utilizes the type IIS restriction enzyme to isolate the terminal 18 nucleotides from both ends of large DNA fragments. The end sequences can then be determined by the massive sequencing of the Illumina platform and mapped back to the reference genome to determine structural variants. The second technology is recently developed by 454 and named the LT paired-end technology. This technology combines the affinity tagging and capture of both ends of $3 \mathrm{~Kb}$ DNA fragments and the 454 pyrosequencing to generate longer end sequences. Both technologies can be applied to either genomic or cDNA fragments to determine structural variants that disrupt or fuse genes and lead to functional changes of their gene products. The resulted datasets will advance the studies of aberrant gene structures and their roles in oncogenesis.

\section{Accomplishments}

Our most significant accomplishment has been to develop a set of experimental procedures and computational tools that can reproducibly generate and analyze massive and low cost end sequences from DNA of selected sizes using the next generation sequencing platforms. We have constructed several $40 \mathrm{~Kb}$ DiTag libraries from non-cancerous and cancerous cells and sequenced them using the 454 technologies. We were able to map more than $80 \%$ of the short 18 bp DiTag sequences to a unique genomic position. The analysis of the mapped DiTag mate pairs has identified many structural variants such as translocations, deletions, inversions, and amplicons in the cancerous genome. A large fraction of these rearrangements and amplicons have been previously identified in independent experiments, which validates the DiTag approach as a fast and cost-effective way of detecting chromosomal aberrations in cancer genomes. To lower the cost further, the same libraries are being sequenced using the Illumina platform.

We have also succeeded in constructing and sequencing several 454 LT paired-end libraries. The low chimera rates and randomness of genome coverage resulted from this technology makes it suitable for identifying structural variants particularly in expressed cDNA populations due to its smaller insert size libraries. As we had expected, this technology is more amenable to a high throughput operation than the DiTag technology. We are now testing this technology for producing large insert sizes. Our hope is to eventually integrate the high throughput 454 paired-end library creation process with the low cost Illumina sequencing to achieve the future goal of analyzing hundreds of cancer genomes to unravel the basis of cancer through discovering genes disrupted by the structural aberrations. 


\section{Life Sciences Division}

Functional Interactomics: Integrating Physical and Functional Interaction Networks Principal Investigator: Gareth Butland

\section{Project Description}

Interactions, both physical and genetic, define the organization of the cell. Physical interactions, such as protein-protein interactions, help us understand how individual polypeptide chains come together to form protein complexes which perform many of the biochemical reactions in a cell. Other types of interactions, such as functional interactions, propose organizational relationships between gene products and protein complexes within the cell. Epistatic (genetic) interactions, one of the most commonly encountered forms of functional interaction, exist between genes which encode proteins involved in parallel pathways or processes. These interactions, whilst not physical in nature, provide information regarding functional redundancy within the cell and help understand the basis of suppressor mutations and synthetic lethality. This LDRD project is designed to develop and implement a methodology capable of screening for functional interactions in $E$. coli on a genome wide scale. The initial technology development phase will be validated using examples of known functionally linked pathways before being applied to uncover novel gene function.

Classical genetic approaches have often combined null alleles in the same strain to assess if a functional relationship exists between two or more genes of interest. These experiments have however usually been restricted to specific biological niches in order to limit the number of null mutations that have to be made sequentially by laborious methods. This project will utilize the recent availability of a complete set of $\sim 4000$ single gene knock-out $(\mathrm{KO})$ strains of $E$. coli. A null mutation in a gene whose genetic interactions are to be screened will then be combined in a pair wise manner with all 4000 mutant strains present in the KO collection and the viability of the strain bearing both mutations assayed. The key step of quickly and easily combining null mutations between strains of $E$. coli in a manner amenable to high-throughput analysis will be developed based on the natural genetic system of conjugation. This will facilitate the rapid screening of thousands of potential functional interactions in a rapid and efficient manner.

\section{Accomplishments}

Over the last year, our focus has been on establishing the technology with which to screen for genetic interactions in $E$. coli, by systematically combining deletion mutations. In collaboration with colleagues at the University of Toronto, we have developed this technology to a stage where it is now ready for use. We have selected a few pairs of genes which have been previously shown in the literature to display functional interactions in order to demonstrate proof-of-principle.

One good example is in the area of Iron-Sulfur cluster biosynthesis where in $E$. coli both IscSUA and SufBCD complexes are known to be functional for cluster formation, which is itself essential for cell viability. Strains bearing deletions of genes present in the ISC and SUF systems have been screened for genetic interactions when combined with mutations from the $E$. coli $\mathrm{KO}$ collection. Potential novel genetic interactions have been observed and we are currently working to complete confirmation of these observations by screening for the reverse interactions. 


\author{
Transcription CoFactor PC4 Interactions with RNA Polymerase \\ and XPG in Transcription-Coupled Repair \\ Principal Investigator: Priscilla K. Cooper
}

Project Description

A major goal of both cancer and aging research is to understand cellular mechanisms for detection and repair of DNA damage that threatens genomic integrity, as well as coordination of these DNA repair processes with transcription, replication, DNA damage signaling, and mechanisms for determining cell fate. Cellular machinery for transcription-coupled repair (TCR) is critical for maintaining transcription by preferential removal of damage that blocks RNA polymerase elongation, and defects in this process result in hereditary disorders with severe clinical phenotypes and early death. However, the complete parts list for TCR and its detailed molecular mechanism are unknown, and the mechanism of TCR has been referred to as "one of the final frontiers in DNA repair research." This project aims to elucidate the mechanism of TCR through characterization of the functional significance of multiple protein-protein interactions of the highly pleiotropic protein XPG, which our evidence suggests plays a key initiating role in TCR. The initial focus was investigation of a proposed role in TCR of a novel player, the transcription cofactor PC4, which globally enhances RNA P II transcription through multiple mechanisms but which we have shown also to play an undefined role in cellular responses to oxidative damage through interaction with XPG. The studies employ an integrated multidisciplinary approach involving coupled cellular studies, biochemical assays, and structural methods.

\title{
Accomplishments
}

Biochemical studies with purified proteins identified direct interactions of PC4 with the three major human DNA glycosylases for repair of oxidized pyrimidines - NTH1, NEIL1, and NEIL2 - and with the TCR protein CSB; all of these also interact with XPG. The PC4-interacting regions of NTH1 and NEIL1 were mapped to facilitate structural studies. To enable cellular studies of the role of PC4 in DNA damage responses, we used RNA interference to knock down the protein in wild type human fibroblasts. Target sequences in PC4 were cloned into a lentiviral construct for inducible shRNA expression under doxycycline control and stable cell lines isolated. These were shown by western analysis to have almost complete knockdown of PC4 after incubation with doxycycline. A comprehensive analysis of sensitivity of the PC4-depleted cells to a variety of DNA damaging agents was carried out. In contrast to hypersensitivity to oxidative damage of $S$. cerevisiae deletion mutants lacking the yeast PC4 homolog, the human cells with deficient PC4 showed no increase in sensitivity to killing by any agent tested.

We therefore focused our efforts to dissect the TCR mechanism and its possible relationship to repair of oxidative DNA damage on other XPG partners. Using human cells expressing a FLAG-tagged version of the NEIL2 glycosylase, we discovered by co-immunoprecipitation and gel filtration that NEIL2 is found in the cell as part of a very large complex with XPG that also includes CSB, the elongating form of RNA polymerase II, the transcription/repair complex TFIIH, and the histone acetyltransferase p300. We showed that this complex occurs in undamaged cells, presumably reflecting responses to endogenous damage, but depends on active transcription. The association of NEIL2 with RNA polymerase requires XPG, and the complex increases upon oxidative damaging treatments. This discovery strongly supports a connection between TCR and base excision repair and provides new tools to understand its mechanism. 


\section{LB06022}

Determine if PIR51 is a Potential Tumor Suppressor Gene Similar to BRCA2

Principal Investigator: David Schild

\section{Project Description}

We have recently identified human PIR51 (Protein Interacting with $\underline{\text { Rad51) }}$ (note - this gene is now named $R A D 51 A P 1$ for $\underline{\text { RAD51 }}$ Associated Protein 1 ) as a new DNA repair gene. Our goal is to further characterize PIR51 and to obtain experimental data (i.e. "proof of principle") to test our hypothesis that this gene functions in the same pathway as $B R C A 2$, a tumor suppressor gene important in breast cancer. Partial depletion of the Pir51 protein by RNA interference (RNAi) sensitizes HeLa cells to a DNA crosslinking agent and to camptothecin (a Topol inhibitor), and causes a high frequency of chromatid breaks following DNA damage. Therefore, cells partially depleted for Pir51 share some properties with BRCA2defective cells, but differences do exist. These differences may be artifacts due to the incomplete depletion of Pir51 or to the use of HeLa cells. Our aim is to test the phenotype of: 1) stable depletion of Pir51 in near-normal human cells, and 2) a pir51 knockout in DT40 cells. We will also test if PIR51 functions in homologous recombinational repair (HRR), as does BRCA2; HRR is an important means of repairing radiation damage, a national health concern due to nuclear power, the potential for a "dirty bomb" and the widespread use of radiotherapy.

Accomplishments

Our major accomplishment during the first year of this grant (FY06) was to determine that BRCA2 and Pir51/RAD51AP1 share several characteristics in common. Like BRCA2, the Pir51/RAD51AP1 protein had been reported to directly interact with the RAD51 protein, and we confirmed this interaction and mapped the region of Pir51/RAD51AP1 that interacts with RAD51, publishing these results in Nucleic Acids Research (see FY06 report). We also determined that Pir51/RAD51AP1, like BRCA2, is directly involved in homologous recombination. These result showing that Pir51/RAD51AP1 functions in HRR were significantly expanded upon in FY07, including genetically showing that Pir51/RAD51AP1 and XRCC3 function in the same epistatic group (i.e. HRR), a group already shown to include BRCA2. In FY07, we did find one major difference between Pir51/RAD51AP1 and BRCA2 that suggested that, although they both function in the HRR pathway, Pir51/RAD51AP1 functions at a later step in HRR than does BRCA2. Our results in tissue culture cells were confirmed biochemically as part of a collaboration with Dr. Patrick Sung's group at Yale. Together with Dr. Sung's group, we recently published our results in Molecular Cell. Our paper, together with a second paper on RAD51AP1 from a Dutch group, were chosen for the cover of this issue of Molecular Cell. (Please note: our submitted JBC manuscript mentioned in the FY06 report was withdrawn in favor of this significantly expanded and improved publication in Mol. Cell.) The following abstract of our Molecular Cell article summarizes the research that was successful finished during FY07.

Homologous recombination (HR) repairs chromosome damage and is indispensable for tumor suppression in humans. RAD51 mediates the DNA strand-pairing step in HR. RAD51 associated protein 1 (RAD51AP1) is a RAD51-interacting protein whose function has remained elusive. Knockdown of RAD51AP1 in human cells by RNA interference engenders sensitivity to different types of genotoxic stress, and RAD51AP1 is epistatic to the HR protein XRCC3. Moreover, RAD51AP1-depleted cells are impaired for the recombinational repair of a DNA double-strand break and exhibit chromatid breaks both spontaneously and upon DNA damaging treatment. Purified RAD51AP1 binds both dsDNA and a D loop structure and, only when able to interact with RAD51, greatly stimulates the RAD51-mediated D loop reaction. Biochemical and cytological results show that RAD51AP1 functions at a step subsequent to the assembly of the RAD51-ssDNA nucleoprotein filament. Our findings provide evidence that RAD51AP1 helps maintain genomic integrity via RAD51 recombinase enhancement. 
Expression Profiling of Radiation and Cancer Susceptibility Genes

Principal Investigator(s): Andrew J. Wyrobek and Francesco Marchetti

\section{Project Description}

The long-term objectives of this research are to identify and characterize the molecular pathways and protein targets that control susceptibilities to low-dose radiation-induced genomic damage and risks for radiation-induced cancers. The goal of this project is to develop a mouse model for identifying radiation-sensitive genes in blood cells to predict the risks of subsequent genomic instability and blood-related cancers.

It is well known that mammalian tissues differ dramatically in their sensitivity to highdose ionizing radiation, but there is little information about the underlying genetic and physiological factors that control susceptibility after low-dose radiation exposures. Under certain circumstances, exposure to low doses confers protection against DNA damage, chromosome damage and cancer, a phenomenon known as radioadaptation. We plan to sample blood from live animals to characterize the gene expression profiles that are unique for radioadaptive exposure regimens and that are predictive for persistent chromosomal damage. This research utilizes several genetic mouse models including those with mutated Tp53, a key controller of the cellular response to ionizing radiation and modulator of cancer risk.

Our findings will elucidate the molecular determinants of the low-dose radioadaptive response to help reduce uncertainties of assessing tissue damage and health risks after low-dose ionizing radiation. It is also anticipated that the mouse model being developed will discover key molecular targets for screening individuals for abnormally clinical sensitivities to radiotherapy and for the development of drug strategies to reduce radiation DNA damage to healthy tissues (radioprotection) and to reduce risks of secondary cancer from radiation therapy (prevention).

\section{Accomplishments:}

We completed a comprehensive survey of radiation-responsive proteins among mammalian species, identifying $\sim 260$ genes whose cellular expressions were modulated after low- to high-dose exposures. Subsets of these genes have promising applications for individual radiation biodosimetry and for identifying individuals who have received only low-dose exposures.

During the past year, we made substantial progress towards developing a mouse model for assessing genomic damage and gene expression changes in blood cells of live animals after radiation exposures. We developed protocols for repeated blood sampling from the leg vein to characterize the effects of low-dose adaptation on DNA damage and repair using single-cell electrophoresis (with blood collected in the first hour after exposure) and gene expression using a transcriptome microarrays (with blood collected within 1 day after exposure). We confirmed that whole-body exposures to a low dose (i.e., priming dose) reduced the magnitude of DNA damage in blood cells after a 2 Gy challenge dose. Using Tp53 null mice, we have obtained preliminary evidence that functional Tp53 may be necessary for the radioadaptive response in mouse blood cells, as previously suggested in our findings of the radioadaptive response in cultured human cells. We have initiated a collaboration to obtain mice carrying various Tp53 mutations with differing sensitivities to radiation-induced cancer to examine their abilities to mount an adaptive response in vivo.

We are also in the process of developing protocols for repeated blood sampling of mice to characterize the persistence of chromosomal damage in blood cells over the period of months after adaptive radiation regimens. 
Tailoring the Self Assembly of Functionalized Biomolecular Building Blocks Principal Investigators: Matthew B. Francis, Phillip L. Geissler, and Jim J. De Yoreo

Project Description

Self-assembling biomolecules could provide efficient scaffolds for the construction of new materials. In addition to possessing nanoscale dimensions overall, the structures they produce often display periodic features that could be used to position objects with ultrahigh resolution. Most of the protein scaffolds reported to date can only form a limited number of geometric arrangements, and little is known about the long range order that these structures can establish. To explore these issues, a systematic and multidisciplined approach has been developed to elucidate the fundamental mechanisms that govern biomolecular self assembly on multiple length scales. The Francis group lent its expertise in protein bioconjugation, in combination with the Geissler group's experience in modeling the self-assembly behavior of complex biological systems. The DeYoreo group at LLNL has played a key role in developing AFM-based imaging techniques to evaluate the structures that are formed in these experiments. These efforts have yielded a versatile experimental platform.

Accomplishments

A central portion of this project has been the creating of patterned surfaces for the welldefined display of monomer disk structures. The Francis and DeYoreo groups have developed two monolayer resists for this purpose that allow (1) the formation of nanoscale patterns on gold substrates using AFM-based nanografting, and (2) the linking of disk structures to the patterned features on these substrates. The most difficult challenge in these studies was the development of a resist layer that did not exhibit non-specific adsorption of the disk structures in undesired locations. With this system in hand, well-defined arrays of functionalized disks can now be created as templates for higher order assembly.

In a second aspect of this work, the Geissler group has developed detailed self-assembly models for the disks using Monte Carlo simulations. By adjusting the interaction strength, angular dependence of adhesion, and the number of molecules that are available for binding, they have predicted that several self-assembly regimes will be observed. They have also shown that by modulating the concentration of the disks, assemblies can be formed, dissociated, and reformed when desired. This could be very useful for the development of systems that can be repaired through this dynamic process.

To link these results with the self-assembly behavior of the disks, the Francis group has developed a series of chemical modification strategies for the introduction of organic molecules on the edges of the disks. They then synthesized dimers that can bind these molecules with known association constants, and thus link the disks together. This modular system thus allows the interaction strengths to be tuned by varying the identity of the organic structures. In ongoing studies, we are correlating the predicted assembly structures with the known binding strength of the attached molecules. As these interactions are characterized and quantified, we anticipate that these studies will yield an unprecedented amount of information about the forces governing higher-order protein self assembly, and thus expand the range of nanoscale architectures that can be generated using biomolecular structures. 
Integration of synthetic nano-materials for high speed, robust, and flexible circuitry

\section{PI: Ali Javey}

\section{Project Description:}

In this project, we will explore integration of cheap and high performance electronic circuitry based on synthetic nanostructured channel materials, such as semiconductor nanowires, on bendable and wearable plastic substrates that are capable of operating in the ultra high frequency (UHF) regime (0.3-3 GHz). The ultimate goal is to demonstrate RF-ID tags based on nanowire thin-film transistors (TFTs).

We will incorporate a systematic research approach in solving the various challenges in order to achieve our objectives. We will: (i) Synthesize high quality nanowire materials. We will mainly focus on $\mathrm{Ge} / \mathrm{Si}$ heterostructure nanowires as they have been shown to exhibit superb electrical properties. (ii) Develop new and improved assembly strategies for assembly of monolayer, well-aligned, and dense films of nanowires on flexible substrates. Such dense nanowire films are highly desired for TFT applications, as they enable high current drives which will directly result in high switching speeds. (iii) Characterize and explore the performance limits of the nanowire TFTs. The cut-off frequency, mobility, and structural capacitance of the TFTs will be characterized and optimized in order to achieve the highest possible performance. (iv) Design and fabricate highly integrated circuitry, such as RF-ID, based on nanowire TFTs.

\section{Achievements:}

The most critical path for realization of high performance nanowire circuitry is to develop strategies for controlled assembly of nanowires on flexible substrates with high uniformity and reproducibility over large scales, and high degree of alignment and order. We have recently developed a roll printing technology for directly assembling semiconductor nanowires on substrates. In this process, nanowires are first grown on a cylindrical roller using CVD, and are then transferred onto a receiver substrate by using a differential roll printing process. The process is highly scalable and allows for controlled assembly of aligned nanowires on virtually any substrate, including flexible plastics, glass, and papers. The nanowire density on the receiver substrate is controlled by surface chemical functionalization, with the highest attained density of $\sim 15$ nanowire/um. Notably, the process is self-limited to a transfer of a single layer of nanowires, without any uncontrolled nanowire stacking.

We are currently using our nanowire printing process to fabricate a wide range of high performance electronic and sensor circuitry. 
Terahertz-Frequency Conductivity and Ultrafast Optical Excitations in Single-Walled Carbon Nanotubes

Principal Investigator(s): Robert A. Kaindl, Yingzhong Ma, Daniel S. Chemla

\section{Project Description}

The goal of this LDRD is to study terahertz-frequency carrier transport and ultrafast electronic processes in single-walled carbon nanotubes (SWNTs). Terahertz (THz) and midinfrared (mid-IR) studies can reveal basic properties, such as plasma frequency, scattering rates, and gaps in metallic nanotubes, and can illuminate the influence of excitonic correlations and screening on the optical resonances of semiconducting SWNTs. Moreover, the relative amounts of homogeneous and inhomogeneous broadening of near-IR absorption lines can be investigated by following the irreversible decay of coherent polarizations. This will provide insight into the role of scattering with electrons, phonons, or excitons, and of higher order processes on the optical response. The insight gained here is critical for optoelectronic nanotube applications, e.g. for fast switches or novel light detectors and emitters at infrared wavelengths.

An array of ultrafast experimental techniques in a broad wavelength range is available to be used in these novel experiments: $(i)$ High frequency transport properties and low-energy excitations of carriers can be determined in a contactless way in metallic and semiconducting SWNTs via ultra-broadband transient THz and mid-IR spectroscopy. (ii) We can utilize degenerate four-wave mixing to study the dephasing of coherent polarizations. Experiments are performed on resonance to the $E_{11}$ and $E_{22}$ absorption lines of semiconducting SWNTs in the near-infrared and visible regions. We investigate SWNTs individually dispersed in micelle or polymer surfactants which is essential to avoid extrinsic nanotube-nanotube interactions.

\section{Accomplishments}

We successfully fabricated and optimized SWNT polymer composites for THz and mid-IR experiments, and characterized them via fluoresence and broadband absorption spectroscopy. Thin polyethylene and cellulose-based samples proved particularly useful for time-resolved mid-IR and THz experiments, and we were able to generate composites with broadband mid-IR transmission that maintain individualized SWNT fluoresence. Tubes grown via the CoMoCAT method were used for selective studies of a narrow species distribution.

Comprehensive measurements of ultrafast broadband mid-IR transmission changes from 4 to $12 \mu \mathrm{m}$ probe wavelength after optical excitation directly uncovered a strong low-energy absorption peak. This feature around $200 \mathrm{meV}$ photon energy can be identified as intra-excitonic transitions between bound excitonic and higher-lying levels. Further studies investigated the mid-IR polarization dependence and the sensitivity to pump wavelength tuning around the $E_{22}$ resonance. Importantly, we investigated the $\mathrm{THz}$ response in detail. Real and imaginary parts of our $\mathrm{THz}$ conductivity spectra provide direct quantitative insight into the conductivity and excitonic nature of the photoexcited state.

Moreover, recent advances in sample preparation enabled measurements of what we believe to be the first photon echo data on individualized SWNTs. The two-pulse photon echo approach was used to unambiguously determine the optical dephasing time of the $E_{11}$ excited state under different temperature, intensity and environment constraints. The three-pulse echo peakshift method was also used to determine the origin of spectral broadening in SWNTs and the $E_{11}$ exciton-phonon coupling strengths. 


\title{
Aging, Disease and the Mechanical Response of Biological Tissues, Specifically Human Bone \\ Principal Investigators: R. O. Ritchie, J. W. Ager III, A. P. Tomsia
}

\begin{abstract}
Project Description
This project enabled a major new effort to be initiated at LBNL with the goal of bridging the gap between the existing clinical understanding of bone fracture and the underlying micromechanical mechanisms of bone failure. Specifically, we targeted how age, osteoporosis, and therapeutic treatments affect the structural properties, e.g., strength and toughness, of bone, and how this translates to the well known increased risk of bone fracture in the aged. We employed quantitative methods that span the relevant hierarchical length scales associated with fracture in bone and have developed new methodologies when required. Measurements at the nano/molecular scales (pico-force AFM, nanoindentation, and vibrational spectroscopies) and microscale (computed hard and soft x-ray tomography) were related to structural behavior at the macroscale (characterized by fracture mechanics testing performed, when possible, in-situ). We examined both human bone, bone from small animal model studies, and also dentin to determine the salient fracture mechanisms. This work has already significantly advanced the understanding of fracture in bone, and the insights developed to date will lead eventually to the development of improved implants and therapeutics.
\end{abstract}

\section{Accomplishments}

Healthy cortical bone has the desirable characteristic that the force required to drive a crack through it increasing with crack extension: this is known as "rising R-curve behavior." Using new fracture testing methods developed in our laboratory, we discovered that in long bones from healthy young adults this effect is larger in for cracks propagating in the transverse, as opposed to longitudinal, direction. Moreover, we determined the mechanism that provides the toughening, namely crack deflection at "cement lines" which are formed during bone's natural remodeling process. Our animal model work enabled us to example changes in fracture properties and mechanisms due to specific drug treatments. For example, a combination of fracture testing combined with $\mathrm{x}$-ray tomography and scanning probe modulus measurements was used to associate undermineralized areas surrounding osteocytes (bone-forming cells) as the possible origin of compromised mechanical strength in glucocorticoid-induced osteoporosis.

This work has demonstrated that a rigorous evaluation of the mechanical performance of bone with an emphasis on the underlying nano/microstructural mechanism is not only possible, but is required to address the public health challenges of increasing bone fracture rates in the elderly. 
Quantifying the Quantum Backaction of a Non-Linear Dispersive Measurement Principal Investigator: Irfan Siddiqi

\section{Project Description}

This research will probe the effect of different types of dispersive measurement on a quantum system such as a single spin $1 / 2$ particle. A dispersive measurement can be realized by coupling the spin $1 / 2$ to an oscillator such that its resonant frequency depends on the state of the spin- up or down. The frequency of the oscillator can be determined by irradiating with an off resonance microwave pulse and studying the reflected or transmitted signal. The act of measurement generates backaction that dephases the quantum state of the spin. This work will focus on dispersive measurements with a non-linear oscillator. The goal is to determine if greater information can be extracted with a non-linear systems, and what quantum uncertainty relations apply in this scheme.

For the project overall, the spin $1 / 2$ system will be realized using a superconducting qubit. The qubit will be coupled to a capacitively shunted classical Josephson junction, which in essence is a non-linear oscillator. The Josephson oscillator will be excited with a $1 \mathrm{GHz}$ probe pulse of varying intensity. For weak pulses, the oscillator is linear and we expect to recover the usual Heisenberg uncertainty relation for the dephasing rate and the measurement time. By injecting more intense microwave pulses, we will study the effect of the oscillator non-linearity on the induced dephasing.

\section{Accomplishments}

The focus of the project is to quantify the influence of a bifurcation amplifier on the measurement of a superconducting quantum bit. The first steps to carry out this experiment have involved the procurement of specialized measurement and fabrication equipment. This project was started late in FY07 to acquire long-lead items to expedite the actual research starting in FY08. Equipment purchases included an ultrasonic wire bonder, a cryogenic pump for a new deposition system, a broadband spectrum analyzer, and a high-speed, 1 gigasample/second digitizer. The cryogenic pump and wire bonder are needed for the fabrication and microwave packaging of the Josephson junction quantum bits. The digitizer and spectrum analyzer will be used in high speed electrical measurements of quantum bit coherence. 
Electron Flow Generated by Gas Phase Exothermic Catalytic Reactions Using MetalSemiconductor Nanodiodes

Principal Investigator: Gabor A. Somorjai

\section{Project Description}

The role of electronic excitation in catalysis at the oxide-metal interface has been explored. The enhancement of catalytic activity due to the presence of oxide-metal interfaces was first suggested by Schwab and others, who performed oxidation of carbon monoxide on $\mathrm{Ag} / \mathrm{NiO}$. The concept of "strong metal-support interaction" was invoked recently for gold-oxide interfaces that exhibit unusual catalytic behavior for small gold particles deposited on titanium oxide or cerium oxide. These experiments suggest that the metal-oxide interface plays an important role in determining chemical reactivity for some classes of oxides (for example, $\mathrm{NiO}, \mathrm{CeO}, \mathrm{TiOx}, \mathrm{NbO}_{\mathrm{x}}$. and $\mathrm{TaO}_{\mathrm{x}}$ ). These oxides have bulk bandgaps of $3-4 \mathrm{eV}$, which form a Schottky barrier at the interface with metal catalysts.

Elucidation of the origin of the metal-support interaction requires measurement of the charge transfer through the oxide-metal interface simultaneously with the turnover rate. We fabricated catalytic nanodiodes that are composed of a thin metal catalyst and an oxide. The electron flow is detected as a chemicurrent if the excess electron kinetic energy generated by the exothermic reaction is larger than the effective Schottky barrier formed at the metal-semiconductor interface. In this project, we aim at utilizing hot charge carriers generated by an exothermic catalytic reaction to detect or even control the chemical reaction and to understand the role of electronic transport of hot electrons through the metal-oxide interface.

\section{Accomplishments}

The influence of physical and chemical properties of $\mathrm{Pt} / \mathrm{TiO}_{2}, \mathrm{Pd} / \mathrm{TiO}_{2}$ and $\mathrm{Pt} / \mathrm{GaN}$ metal-semiconductor Schottky diodes on the yield of collected hot electron flow (number of hot electrons per product molecule) was investigated. We measured both the chemicurrent (electron flow) and chemical turnover rate during oxidation of carbon monoxide (at pressures of 100 Torr of $\mathrm{O}_{2}$ and 40 Torr of $\mathrm{CO}$ in the $373 \sim 513 \mathrm{~K}$ range) using reaction systems equipped for simultaneous reaction rate and current measurements. The chemicurrent was found to be correlated with the turnover rate and can be used to detect the turnover rate for three diodes. Thermoelectric current was observed in the presence of $\mathrm{O}_{2}$ or $\mathrm{CO}$ gas in the absence of catalytic reaction. We found that the metalsemiconductor interface structure (roughness, grain size, and step-terrace) is important in controlling the magnitude of chemicurrent yield.

We fabricated nanoparticle-nanodiode hydrid system that is composed of Pt metal nanoparticles (size 3-13nm), Au thin film (2nm thick), and $\mathrm{TiO}_{2}$. Interface between $\mathrm{Au}$ and $\mathrm{TiO}_{2}$ forms the Schottky barrier that has the energy barrier of $1.0 \mathrm{eV}$. The overall thickness of metal assembly (nanoparticles and Au thin film) is comparable to the electron mean free path, resulting in the ballistic transport of hot electrons through the metal. The chemicurrent and chemical reactivity on nanoparticles with various capping agent (citrate, PVP, Ctab, thiol and amine) and sizes (3-14 nm) were measured during catalytic $\mathrm{CO}$ oxidation. We found the chemicurrent and chemical reactivity significantly depends on the types of capping layers in a similar manner. 
Studies of Quantum Antiferromagnetism in Two-Dimensional Triangular Lattices Using Ultracold Atoms

Principal Investigator: Dan Stamper-Kurn

\section{Project Description}

The long-term goal of this LDRD-sponsored project was to lay the groundwork for using ultracold atomic gases to address the open question of quantum magnetism in condensed materials, focusing specifically on antiferromagnetism in a two-dimensional anisotropic triangular lattice. This configuration was chosen for being a tractable system for studying conventional antiferromagnetic behavior while also being amenable to modifications that could yield exotic topologically ordered quantum phases. Realizing such phases will allow for detailed studies of novel concepts in materials physics.

Initially, the aim of the project was to adapt an existing experimental apparatus to the study of quantum magnetism. This adaptation involves redesigning and rebuilding the ultrahigh vacuum chamber, and setting up the optical system to deliver laser light which will produce a spatially periodic potential in which to trap cold atoms. We also sought to develop theoretical ideas and experimental methods for producing and probing the magnetic states of ultracold atomic materials.

\section{Accomplishments}

We report three accomplishments for FY07. First, we have employed magnetizationsensitive imaging of ultracold atomic gases to demonstrate a spatially resolving magnetometer of unprecedented sensitivity. This demonstration is the first application of ultracold quantum gases to a task of direct scientific and technological importance. The demonstrated sensitivity of this magnetometer, $0.5 \mathrm{pT} / \mathrm{Hz}^{1 / 2}$ at unity duty cycle and over a measurement area of $120 \mu^{2}$, is already 60 times better than that of state-of-the-art SQUID magnetometers. Aside from presenting clear advantages for "conventional" studies of materials, e.g. measuring properties of weakly magnetic thin-film or bulk materials, such a magnetometer may also serve as a probe of magnetic properties in ultracold atomic systems in which quantum magnetism is simulated.

Second, we added to our experimental repertoire the ability to differentially tune the energies of spin states of ultracold $\mathrm{Rb}$ atoms using off-resonance microwave radiation. This capability is being used in present studies of quantum phase transitions in ferromagnetic spinor Bose-Einstein condensates.

Third, we have completed the design of a new vacuum chamber and optical system which will physically and effectively separate experimental functions, namely the production of ultracold atomic gases and their use for lattice-based quantum simulations, so as to allow for more sophisticated and robust experiments. Major components of this system have been purchased and two graduate students, Sabrina Leslie and Jennie Guzman, have been supported to conduct this work 


\title{
Hierarchically Nanostructured Systems for Solar Energy Hydrogen Production
}

\author{
Peidong Yang (MSD), Don Tilley, Chris Chang (CSD)
}

\section{Project Description:}

A sustainable hydrogen economy would require using renewable energy (solar energy) and a suitable feedstock (e.g., water). Energy from the sun is abundant, renewable, and can be utilized without negative impacts to the environment. In particular, solar energy may provide low-cost and efficient routes to hydrogen. This will require the invention of new photoactive materials that accomplish the combined tasks of light harvesting, charge separation, and compartmentalized chemical transformations. These new materials are expected to incorporate many of the design principles that operate in biological photosynthetic systems. We propose to develop synthetic, photocatalytic materials based on nanostructures that allow the spatial arrangements of active components, and the 'traffic control' of chemical reactants, intermediates, electrons and products. The proposed system starts with a light harvesting semiconductor core-sheat11 nanostructure array embedded in a thin layer of porous media for proton transfer/supply. The nanostructures will be asymmetrically functionalized with catalytic active sites for water oxidation and hydrogen production from protons. The precisely controlled arrangement of active components within such hierarchical nanostructures is the key to the effective coupling of various processes including light absorption, charge separation, water activation, and hydrogen production.

\section{Accomplishments:}

Core/shell nanowires of a certain composition have been asymmetrically functionalized with catalytic active sites for water oxidation and hydrogen production to allow effective charge separation and light absorption along the wire. Arrays of vertical nanowires have been synthesized using a vapor-liquid-solid process, followed by conformal coating with a second material using atomic layer deposition (ALD). Further processing was done to make a final array of core-shell nanowires asymmetrically decorated with two different catalysts.

We have discovered certain metal complexes that are competent catalysts for converting protons to hydrogen under reducing potentials. The catalysts function in several types of mixed aqueous/organic or organic media with various protic acid sources. Details of the results will be reported in upcoming publications. Post-project, we are currently performing experiments to fully analyze the speed and stability of these catalytic systems. 
New Experimental Program to Deduce $(\mathrm{n}, \mathrm{f})$ Cross-Sections for Advanced Fuel Cycle Studies

\author{
Roderick Clark
}

Project description:

This is a new research effort to determine basic nuclear physics data for Advanced Fuel Cycle (AFC) studies. Sustainable, safe nuclear energy has been identified as an important national need and the necessary basic research is part of the DOE mission. We are attempting to determine cross-sections for neutron-induced fission of actinides involved in fast reactor fuel cycles. Existing data often show significant discrepancies and do not extend to the higher neutron-energy range (up to $\sim 20 \mathrm{MeV}$ ) found in fast reactors. The goal is to develop novel surrogate reactions to deduce the relevant cross-sections to unprecedented accuracy $(<10 \%)$ over a broad neutron-energy range $(0.2$ to $20 \mathrm{MeV})$. The surrogate reaction method uses a light-ion reaction to determine the decay probabilities of a compound nucleus of interest. The formation probability can be modeled accurately. By combining the calculated formation probability with the measured fission probability the desired $(\mathrm{n}, \mathrm{f})$ cross-section can be deduced. We use light-ion beams, accelerated by the 88-Inch Cyclotron, which are incident on actinide targets. Charged particles and fission fragments are detected in the STARS Si-detector array in order to determine the required fission probabilities.

Accomplishments:

Over the last year a strong LBNL/LLNL collaboration has formed to study various aspects of surrogate reactions. We have performed a benchmark experiment which has shown that surrogate reactions measured in ratio such as ${ }^{234} U(\alpha, \alpha$ 'f $)$ / ${ }^{236} \mathrm{U}\left(\alpha, \alpha^{\prime} \mathrm{f}\right)$ reproduce, to better than $10 \%$, the well established values of ${ }^{233} \mathrm{U}(\mathrm{n}, \mathrm{f})$ / ${ }^{235} \mathrm{U}(\mathrm{n}, \mathrm{f})$ measured in direct reactions. This proves the validity of our approach. We have also collaborated on initial experiments using the $\left({ }^{18} \mathrm{O},{ }^{16} \mathrm{O}\right)$ reaction.

The main focus for LBNL has been to study the $\left({ }^{3} \mathrm{He}, \mathrm{t}\right)$ reaction as a potential surrogate. This will be an important reaction since it allows one to use relatively stable even-even targets to deduce information about odd-Z isotopes (e.g., Np and Am). We have indirectly determined the ${ }^{237} \mathrm{~Np}(n, f)$ cross section over an equivalent neutron energy range from 10 to $20 \mathrm{MeV}$ using the surrogate reaction ${ }^{238} \mathrm{U}\left({ }^{3} \mathrm{He}, t f\right)$. A self-supporting $\sim 761 \mu \mathrm{g} / \mathrm{cm}^{2}$ metallic ${ }^{238} \mathrm{U}$ foil was bombarded with a $42 \mathrm{MeV}{ }^{3} \mathrm{He}^{2+}$ beam from the 88-Inch Cyclotron at Lawrence Berkeley National Laboratory (LBNL). We have compared the results of this work with direct measurements of the ${ }^{237} \mathrm{~Np}(n, f)$ cross section data, Evaluated Nuclear Data File (ENDF/B-VII.0), and Japanese Evaluated Nuclear Data Library (JENDL 3.3). The cross section data of this work follow the same trend with a maximum discrepancy of $\sim 5 \%$ when compared with the ENDF/B-VII.0 dataset. An expected discrepancy between the direct and indirect ${ }^{237} \mathrm{~Np}(n, f)$ cross section data due to pre-equilibrium effects is found to be smaller than model predictions in this energy range. A paper describing this work is in preparation. 


\section{Physics Detector and Sensor Technologies Applied to Geological and Geophysical Applications at DUSEL Principle Investigators: Kevin Lesko and Joe Wang}

\section{Project Description}

The purpose of this project is to develop solutions to R\&D issues, including significant societal problems, utilizing the new opportunities provided by the Deep Underground Scientific and Engineering Laboratory (DUSEL). Partnerships between Earth Scientists and Physicists will be developed to investigate underground carbon remediation techniques, understand heat flow and origins in the earth and research geothermal energy extraction, and develop novel detector components and materials for underground research. The $1^{\text {st }}$ activity researches underground carbon sequestration, developing techniques for transporting, and monitoring $\mathrm{CO}_{2}$ and understanding long term sequestration by characterizing the site and developing monitoring tools and techniques. The $2^{\text {nd }}$ effort is aimed at understanding underground heat flow and the origins of terrestrial heat. DUSEL offers a laboratory setting to understand heat flow. The origins of terrestrial heat will be investigated by developing concepts for geoneutrino detectors. This effort will investigate sustained geothermal extraction in DUSEL to understand the design and development of geothermal fields. The $3^{\text {rd }}$ topic will investigate detector materials with a focus on ultra-low contamination of radioactive species.

We draw on Earth Sciences experience in characterizing the underground, coupled processes with $\mathrm{CO}_{2}$ sequestration and Physics instrumentation, Monte Carlo simulations, and sensors. The multi-divisional team, together with established DUSEL collaborations, work together to quantify the distribution of radioactivity in the rock, correlate the heat flows with crustal $U$ and Th, construct models, and evaluate the leakage and storage of gaseous and liquid fluids. DUSEL test sites will be located to conduct in situ experiments for fluid flow, chemical transport, and heat transfer, measure the migration of injected $\mathrm{CO}_{2}$.

In comparison with solar radiation and neutrino flux from fusions in the Sun, we have modest and heterogeneous heat flow and geoneutrinos from fission in the Earth's crust and the core. It is a great challenge to quantify heterogeneous processes underground. The Homestake, with its relatively low background located away from the volcanic arcs, offers opportunities for fundamental understanding of flow and isolation potential of the Earth.

\section{Accomplishments}

Carbon Sequestration: We located candidate test sites along sandline boreholes to develop procedures and techniques to characterize the sequestration test bed and to monitor the evolution of injected $\mathrm{CO}_{2}$, including phase transition, migration and dispersion. We have begun research on sensors, working towards the integration of a new family of gas micro-sensors.

Heat Flow and Origins: We developed plans to characterize and monitor heat flow in DUSEL, as well as developed experimental programs required for these characterizations. This included producing detailed Monte Carlo simulations of a geoneutrino detector. DUSEL offers the opportunity to characterize and prepare a thermal extraction test bed. Rock fracturing and bed preparation are now being researched and ultimately to be characterized in detail.

New Materials and Sensors: Next generation detectors require special materials including ultra-low background materials, ultra compact and ultra-pure electronics for detectors, and remote sensor arrays. We researched recent developments and identified candidate technologies for ultrapure materials and producing detectors from these materials. 
Improved Spectroscopy of Weakly Bound States in Nuclei

Larry Phair

\section{Project Description:}

Future nuclear structure measurements will take place at the drip lines of very neutron-rich and proton-rich nuclei. These nuclei will be identified by detecting the last particle stable isotope and the spin and parity of its ground state and low excited states, if any. By studying states at high excitation energy now (i.e., the unpopulated drip line states), we can explore in a novel way the single particle, pairing, and vibrational properties of states more commonly studied near the Fermi surface in ordinary nuclei.

Our goal is to demonstrate the feasibility of studying the future drip line single particle and collective states, by populating these states which lie about 6-7 MeV above the Fermi surface in ordinary nuclei.

\section{Accomplishments:}

During the past year we acquired digital signal processing (DSP) electronics that will allow us to expand our coverage of charged particle and gamma ray detection. The DSP electronics can be used with silicon and germanium detectors.

We have studied the spectroscopy of light, neutron rich nuclei by populating them with fusion evaporation reactions. The fusion-evaporation reaction ${ }^{9} \mathrm{Be}\left({ }^{11} \mathrm{~B}, 2 \mathrm{p}\right)$ was used to populate excited states in ${ }^{18} \mathrm{~N}$. New gamma transitions were added to its level scheme. The mean lifetime of the first excited state was measured and its transition rate to the ground state was determined. Shell model calculations were used to investigate the lowlying configurations of ${ }^{18} \mathrm{~N}$ and in the $N=11$ isotones ${ }^{17} \mathrm{C}$ and ${ }^{19} \mathrm{O}$. It was found that the role of the proton-neutron interaction is important in determining the ground state and low-lying excited state properties. The ground state spin inversion in these isotones is attributed to the increased importance of the quadrupole relative to the pairing interaction. This work has been submitted to Physical Review $C$ for publication.

The lifetime of the first excited $2+$ state in ${ }^{16} \mathrm{C}$ has been measured with the recoil distance method using the ${ }^{9} \mathrm{Be}\left({ }^{9} \mathrm{Be}, 2 \mathrm{p}\right)$ fusion-evaporation reaction. The mean lifetime was measured to be 11.7(20) ps corresponding to a B(E2) of (1.73(30) W.u.), consistent with other even-even closed shell nuclei in the vicinity. Our result does not support an interpretation for "decoupled" neutron and proton shapes (the recent RIKEN work). These results have been submitted for publication to Physical Review Letters.

We explored the possibility of studying ${ }^{26} \mathrm{Ne}$ via the reaction ${ }^{11} \mathrm{~B}\left({ }^{18} \mathrm{O}, 3 \mathrm{p}\right)$. An excitation function was measured and analysis is almost complete.

Direct reactions were measured using the light-ion beams from the 88-Inch Cyclotron. The reactions ${ }^{171,173} \mathrm{Yb}(d, p \gamma)$ were performed to populate single particle states in ${ }^{172,174} \mathrm{Yb}$ both near the surface and at higher excitation energies as well. States near the surface were identified with both charged particles and coincident gamma rays. The detected charged particles were used to establish the excitation energy of the nucleus. 
Hyperons in Polarized Proton Collisions and the Origin of the Nucleon Spin Principal Investigator(s): Ernst Sichtermann

\section{Project Description}

The aim of this project is to delineate the flavor composition of the quark and anti-quark spin contributions to the nucleon spin by determining the longitudinal spin transfer of hyperons and anti-hyperons produced with large transverse momenta in collisions of polarized proton beams at the Relativistic Heavy Ion Collider (RHIC). If successful, this will give the STAR experiment at RHIC unique access to the spin distributions of strange quark and anti-quarks in the polarized proton. It will provide also a novel way to access the spin distributions of the up and down (anti-)quarks.

We will develop the phenomenology of (anti-)hyperon polarization in polarized proton collisions, extending our earlier exploratory study of the (anti-)Lambda hyperon, perform a proof-of-principle spin analysis of hyperon data collected with STAR, and develop an experimental concept to efficiently trigger on (anti-)hyperons with large transverse momenta.

\section{Accomplishments}

The most significant accomplishment to date in this project has been to systematically map the phenomenology of the Lambda, Sigma, and Cascade antihyperons. The Sigma is found sensitive to the spin distributions of the up and down antiquarks, and the Cascade to that of the strange anti-quarks. A paper has been submitted for publication.

We have completed also a proof-of-principle analysis of longitudinal spin transfer for Lambda and anti-Lambda data collected with a beam-collision trigger in STAR. A control sample of the more abundantly produced and spinless K-mesons was used to assess systematic uncertainties in the measurement. Preliminary results were presented at the SPIN-2006 conference and will appear in the conference proceedings. A paper is in preparation.

We are analyzing data collected with more advanced triggers to extend the covered range of transverse (anti-) hyperon momenta, and are in the process of investigating new trigger concepts. 
Development of a Low-Energy, High Current Astrophysics Accelerator

Principal Investigators: Paul Vetter, Daniela Leitner, Matthaeus Leitner, Damon Todd

Project Description

We are developing a high current accelerator with which to measure nuclear fusion reaction cross sections for main sequence stars. Precise determination of these cross sections are needed to fully model stellar and galactic evolution, the energy balance of stars, the isotopic composition of solar system and galactic material, and to interpret current and planned solar neutrino oscillation experiments. To measure several hydrogen and helium-burning reaction cross sections, the beam energy must be as low as possible to mimic stellar burning conditions and to measure near-threshold resonant nuclear contributions. Below $100 \mathrm{keV}$, measuring the low event rates requires intense beams $(>100 \mathrm{~mA})$, high density targets, high-efficiency event counting, and good background rejection. We are designing an accelerator, ion transport system, high-flow gas jet target, target region, and detector system to meet these requirements.

Accomplishments

To deliver ion beams with $100 \mathrm{~mA}$ currents and 50-300 keV energies to a gas jet target with sub-millimeter diameter, beam emittance growth must be minimized. To reduce space charge effects on beam emittance, beam potential screening by secondary electrons will be used. The electrons are introduced through collisions of the beam ions with background gas, and they can be confined by the beam's potential, effectively reducing it. However, this shielding cannot be maintained across the primary acceleration column. We have designed a column which has a gap length that can be adjusted for each energy. We have developed a computer model incorporating two Boltzmann electron distributions for screening - before and after the gap, and have used the simulation to optimize gap geometry over the wide range of accelerating voltages. Simulations have shown that the gap diameter and length should be kept as small as possible to reduce emittance growth during acceleration. Reducing the length and diameter reduces the size of the unneutralized region, and the emittance growth due to unneutralized transport far outweighs growth due to induced aberrations. With the adjustable acceleration column, simulations show it is possible to deliver sub-millimeter beams to a gas jet target over the full energy range.

We have studied space charge neutralization using multi-grid retarding potential analyzers in the transport system for the VENUS ion source. Initial studies show that at normal operating pressures $\left(10^{\wedge}-8 \mathrm{mbar}\right)$, there is little electron shielding. We have measured effective beam potentials to $10^{\wedge}-6$ mbar, and conclude that pressure must be higher to reach $90 \%$ neutralization.

We have begun modeling the supersonic gas jet target. The design is difficult since the ion beam final focus region is constrained by the focal properties of the final beamline elements and the size of high-efficiency detectors. We have begun finite element analysis modeling of the supersonic gas jet flow to arrive at a satisfactory design for the supersonic gas jet nozzle and gas flow operating parameters (temperature and pressure). 


\author{
Research Tools for the Conversion of Cellulose to Ethanol: Structural Studies of Cellulose \\ Synthesis \\ Principal Investigators: Paul D. Adams, Manfred Auer, and Chris Somerville
}

\title{
Project Description:
}

The goal of this work is to apply medium to high resolution imaging methods to determine the detailed mechanism of cellulose synthase (CESA); the molecular machine responsible for the synthesis of the cellulose component of the plant wall. Specifically, we are using X-ray crystallographic, electron microscopy, and tomographic methods to determine the stoichiometry of the CESA complex, its position in the cell membrane, its relationship to the nascent cellulose fibrils, and ultimately the atomic resolution structure of the CESA active site. The information generated will be critical to the development of modified plants as the source of cellulose for ethanol production.

Three groups are collaborating to generate advances in our understanding of cellulose synthesis. CESA proteins from Arabidopsis and microbes such as E. coli, are being purified and genetically labeled by the group of Chris Somerville. These proteins are being studied using crystallographic methods by the group of Paul Adams. Plant and microbial samples are being studied using electron microscopy tomographic methods by the group of Manfred Auer.

Accomplishments:

Our most significant accomplishments have been 1) to explore various sample preparation methods for electron tomography of CESA, 2) to contribute to a model of CESA by studying the effect of mutants on the cell wall architecture and 3) to express and purify domains of the CESA protein for structural studies.

We have developed techniques for the expression of the N-terminal domain and central domain of cellulose synthase from Arabidopsis in E. coli. It has been possible to express the Nterminal domains in large fermentors, which has provided the yield of protein required for crystallographic structural studies. The purification procedures for these domains have been developed further to optimize protein yield and purity. The automated crystal screening equipment at the Berkeley Structural Genomics Center (BSGC) has been used to search for initial crystallization conditions of the CESA3 N-terminal domain.

We have succeeded in developing a protocol that faithfully preserves the molecular ultrastructure of plant cells. The level of preservation of the cell wall matches what can be accomplished by cryo-sectioning, as judged by the similarity in texture of the cell wall, when viewed in projection as well as the absence of aggregation or extraction artifacts typically encountered in conventionally prepared samples. We have also been able to preserve and visualize the underlying microtubule network that is believed to guide the cellulose synthase, which now forms the basis of electron tomographic analysis of the 3D architecture of the cellulose synthase. The sample preparation methods we have developed will be directly applicable to the plant biology work we are pursuing at the Energy Biosciences Institute.

The excellent preservation we have obtained made it possible to quantitatively address cell wall thickness and density as a function of different CESA mutants, leading to the conclusion that the primary complex requires three unique types of components, CESA1-, CESA3- and CESA6-related, for activity, with CESA6-related CESAs being partially functionally redundant (Persson et al. 2007 PNAS). 
Cellulosomes and a Metabolic Pathway for Hydrocarbon Production

Principal Investigators: Jamie H. D. Cate, Michael A. Marletta, and Jay Keasling

\section{Project Description}

Derived from solar energy, cellulose is the most abundant biomolecule on the planet and, therefore, could be used to generate renewable energy sources that might replace the use of oil. At present, there are no efficient or economical means for converting cellulose into useful biofuels. This is primarily due to the insoluble and heterogeneous nature of cellulose sources, i.e. plants. In the biosphere, cellulose in plant debris is efficiently degraded by some anaerobic microorganisms, which use the products for growth. It may, therefore, be possible to exploit the mechanisms used by these bacteria to break down cellulose to provide feedstock for biofuel production.

The molecular machinery responsible for the degradation of cellulose is found in supramolecular, multi-enzyme complexes called cellulosomes that can reach many MDa in size. One goal of the present project is to develop an experimental system to determine mechanistically how cellulosomes efficiently degrade cellulose. At present, it has not been possible to probe the structure and function of intact cellulosomes, due to their very large size and heterogeneous composition in many organisms. Furthermore, since the substrate for cellulosomes is a solid, the cellulosome is not easily studied by standard enzymological approaches. We propose to develop a model system of the cellulosome that will enable us to study its enzymatic properties at a fundamental level.

Once simple sugars have been produced from plant biomass, these sugars will need to be chemically converted to useful liquid fuels. Attractive biofuel alternatives, i.e. long-chain alcohols, aldehydes and alkanes, may be produced microbially using the tools of synthetic biology. Longer chain fuels from microbes will eliminate a significant amount of energy required for processing (distillation, crushing, etc.). Fatty acid biosynthesis is a metabolic pathway that can produce the precursors to long chain alcohols, aldehydes and alkanes by reduction of free fatty acids. This pathway is attractive for biofuel production because fatty acids are commonly used as energy storage molecules in many organisms, such as yeast. Some yeasts have been shown to accumulate fatty acids to a large percentage of their weight, indicating that it is possible to shunt significant amounts of energy into building fatty acids. We are therefore using S. cerevisiae for production of fatty acids.

\section{Accomplishments}

We have cultured a Clostridium species in the lab that produces soluble cellulosomes less than $1 \mathrm{MDa}$ in size. We characterized the components and activity of these cellulosomes by various protein biochemical approaches. We have also developed a transformation protocol for the Clostridium strain that will allow us to carry out genetic manipulations of the cellulosome for biochemical and biophysical studies.

As an initial attempt to increase fatty acid biosynthesis in $S$. cerevisiae, we have overexpressed the FASs using common high-copy vectors. Strains were analyzed by GC-MS for differences in the various saturated and unsaturated fatty acids synthesized. The lab also worked to optimize carbon flux in these strains. 
The Statistical Dynamics of Protein Evolution

Principle Investigators(s): Gavin E. Crooks

\section{Project Description}

The goal of this project is to understand the dynamic evolution of proteins on various time scales, and to create the next-generation algorithms for the automatic reconstruction of protein evolution from protein sequences. This requires a mathematically elegant, computational efficient and biological realistic model of protein sequence evolution over long time scales; an efficient Monte-Carlo algorithm for sampling from the posterior distribution of protein histories; and reliable benchmarks to validate the authenticity of the reconstructed histories. Three deficiently with existing algorithms will be addressed; the simulations inferences of sequence alignment and protein evolution using a consistent algorithm; the higher level evolution of proteins through domain reorganization; and the computational challenges that must be faced when considering the vast and rapidly growing size of the protein sequence database.

\section{Accomplishments}

We have been investigating statistical relationship between the amino acid sequence of a protein and it's structure, and how these relations are preserved during evolution. We have found that amino acids that are neighbors in the structure, but distant on the chain are almost uncorrelated. This implies that proteins are held together by subtle diffuse interactions between many residues, and not by strong direct pairwise interactions, as is often assumed.

These insights into protein sequence evolution allow the construction of a novel heterogeneous profile of how the sequence of proteins change with time. In turn this allows construction of principled and effective profile-profile sequence alignment algorithms . A surprising corollary is that many existing, widely used empirical homology scores are actually approximations on one another and of the heterogeneous profile model, modulo important scaling constants. This may explain why it has been so hard to demonstrate that any one empirical score is inherently superior to any other empirical score. 
Light Regulation and Visible Light-Driven Water Oxidation in Engineered Photosystems Principal Investigators: Heinz Frei, Vittal Yachandra, Phill Geissler, Chris Voigt, Dan Fletcher, Kris Niyogi

Project Description:

The generation of solar fuels by the direct conversion of light energy to fuel molecules in a macromolecular assembly is an attractive goal, yet one that has not yet been achieved in an efficient practical system. Among the major gaps are (1) the fragility of 'soft' organic components, especially those for light harvesting under intense solar light irradiation, and (2) the efficient coupling of water oxidation and proton or carbon dioxide reduction half reactions in an integrated fuel generating system. The goal is to develop solutions to overcome these two major scientific hurdles.

Enhancement of the durability of soft components will be explored by introducing light regulation and protection in two types of solar conversion systems: a minimal biomimetic system in which reorganization of photosynthetic particles in response to light intensity change will be achieved by an actin polymerization sub-system. In parallel, transferable regulation of light harvesting will be explored by introducing the polypeptide PsbS as self-contained photo-protective unit in photosynthetic cyanobacteria that lack major short-term regulation mechanisms. For integration of photocatalytic components for water oxidation and carbon dioxide reduction into a solar fuel system, an approach inspired by natural Photosystem II is proposed that consists of the stepwise assembly of biomimetic polynuclear units as active sites in a nanoporous oxide scaffold.

Accomplishments:

We have developed a technique to encapsulate proteins and micron-sized particles within vesicles by deforming a planar lipid bilayer. Specifically, we demonstrated encapsulation of actin, associated proteins, and chloroplast-sized polystyrene beads (model for photosynthetic particles). This accomplishment provides a platform for studying spatial organization of components under the influence of changing light intensity. We have generalized our computational model for the statistical mechanics of actin networks to include filament polymerization and cross-linking. This development opens doors to exploring the far-from-equilibrium network dynamics involved in actinbased spatial organization.

Plants regulate photosynthetic light harvesting by a mechanism called $\mathrm{qE}$ that dissipates excess absorbed light energy and protects against photo-oxidative damage. qE requires a specific protein, PsbS. We have transferred the PsbS protein from a plant, Arabidopsis, to a unicellular green alga, Chlamydomonas, which in its native form has a poor light regulation system. Several transformants were obtained that exhibit higher levels of $\mathrm{qE}$. In order to investigate the mechanism of regulation in Chlamydomonas, we carried out the first ultrafast pump-probe experiments on live wild-type cells and discovered that it has a minor complex-based-regulation mechanism despite the fact that it does not contain PsbS protein. This newly discovered process is under investigation.

A biomimetic $\mathrm{Mn}$ dimer complex ((bpy $\left.)_{4} \mathrm{Mn}_{2} \mathrm{O}_{2}\right)$ for water oxidation has been successfully loaded into a nanoporous silica scaffold with structure and $\mathrm{Mn}$ (III)Mn(IV) oxidation state intact (EPR, EXAFS, FT-Raman). We have been able to couple the complex to single chromium centers embedded in the nanopore silica surface that act as photon-driven electron pumps. Visible lightinduced electron transfer from the $\mathrm{Mn}$ dimer core to the $\mathrm{Cr}(\mathrm{VI})$ chromophore was demonstrated by FT-Raman and EPR spectroscopy, with all initial and final Mn and Cr oxidation states directly observed. This is the first example of photo-driven pumping of electrons from a biomimetic water oxidation complex in a nanoporous scaffold using a molecular charge-transfer unit. The flexibility of selecting metal and oxidation state of the unit is the key for the efficient coupling of redox catalysts. 
Cooperation of Biochemical and Mechanical Signals in Regulating Cell Fate Decisions During Tissue Morphogenesis

PI: David Schaffer, with Mina Bissell and Adam Arkin

Postdocs: Chinmay Pangarkar and Carole Berruyer-Pouyet

Project Description:

We are developing an interdisciplinary approach that unites multiple investigators with different disciplines from LBNL and UCB to study how cells interact with each other and their microenvironment during the processes of tissue regeneration and repair. This effort is bridging mathematical modeling, quantitative analytical biology, biomaterials development, and high impact biological systems in an integrated effort to advance our understanding of a very complex and important area of biology.

We focus on two central model systems that have many features in common, but a number of fundamental differences: (1) the branching and "invasion" of the mammary tree into the mammary fat pad, and (2) the migration and differentiation of neural stem cells (NSCs) to form new neuronal circuits in the adult brain. We investigate how the mechanical and biochemical signals that comprise the cellular microenvironment cooperate to regulate cell functions such as proliferation, migration, invasion, and differentiation. In particular, we are investigating the roles played by the biochemical signaling system Eph/Ephrin in conjunction with matrix mechanical stiffness in mouse mammary cell and NSC proliferation and differentiation.

\section{Accomplishments:}

In breast tissue, we find that expression of two Eph receptors is induced during puberty, when extensive ductal branching occurs. To assess the effect of Eph activation on cell proliferation, a process of fundamental importance to branching morphogenesis, we have used both Eph4 and ScP2 mouse mammary epithelial cell lines. We have verified that these cells express the transcripts and the proteins of both receptors. In all cases, the proliferation of both Eph4 and Scp2 cells were inhibited by the addition of the clustered ephrin ligands in the cultured media. Further, we demonstrated that this decrease of growth induced by the ephrin ligands is correlated with an inhibition of the MAPK pathway. To test the effect of Eph activation on the differentiation process, we currently are collecting data using physiologically relevant $3 \mathrm{D}$ culture assays of mammary cell lines as well as mouse primary organoids.

In NSCs, ephrins B2 and B3, as well as Eph A2, were shown to exert a strong proliferative effect, increasing the cell number threefold over control. These findings show that activation of the Eph B family of receptors, and the ephrinA family of ligands leads to increase in cell proliferation. On the other hand, when cell fate was examined using immunofluorescent molecular markers for differentiated cells, ephrin B2 was observed to promote neuronal differentiation to a considerable extent. Taken together, results from in vitro experiments allow us to conclude that Eph-Ephrin system plays a significant role in determining the fate of NSCs.

Future objectives include validation of these findings in vivo, an investigation of the relevant intracellular mechanisms, analysis of the role of matrix stiffness, and mathematical modeling of both neural and breast cell function. In addition, we will pursue a NIH training grant funding mechanism in the near future. 


\section{Physics Division}

\section{Advanced Monolithic Si Pixel Sensors for the ILC Principal Investigator: Marco Battaglia}

The R\&D program addresses the development of Si pixel sensors of new concept for the detection of ionizing particles with emphasis on application at an $e^{+} e^{-}$linear collider. The anticipated physics requirements push the detector specifications to new levels. This requires R\&D which is complementary to that driven by LHC and SLHC and reaches out to potential applications beyond experimental particle physics, such as imaging, electron microscopy and FELs beam diagnostics. The technology of choice for this program is monolithic CMOS pixel detectors, which integrate readout and data processing capabilities into the same silicon substrate used for detection and offer unprecedented opportunities in terms of position resolution and readout speed within a minimal amount of material.

The final year of the project has been devoted to tests of the LDRD-2 chip, which features $20 \times 20 \mu \mathrm{m}$ pixels with in-pixel charge storage to baseline subtraction. The sensor has been designed for fast readout and has been successfully tested at readout frequencies up to $25 \mathrm{MHz}$. Good signal-to-noise ratio has been measured with $1.5 \mathrm{GeV} e^{-} \mathrm{s}$ and $120 \mathrm{GeV}$ protons. The successful pixel cell of the LDRD-2 chip has been used as starting point for the third chip in produced in the framework of the LDRD program. The LDRD-3 chip adds a row of 5-bit successive approximation, fully differential ADCs located at the end of the pixel column. Each ADC, which matches the pixel footprint and measures $20 \mu \mathrm{m} \times 1 \mathrm{~mm}$, has been designed for minimal power consumption $(\leq 0.4 \mathrm{~mW} / \mathrm{ADC})$. Tests of the ADCs show that the devices are functioning and meet the specifications.

A complementary approach to the construction of monolithic pixel sensors using consumerdriven Si fabrication technologies has led to the design and fabrication of a pixel detector test structure in silicon on insulator (SOI) technology. SOI allows to fabricate CMOS circuits on a thin Si layer, electronically insulated from the rest of the wafer. The isolation of the electronics from the detector volume offers clear advantages. First, both nMOS and pMOS transistors can be built, without disturbing the charge collection. Further, the detector wafer can be biased, thus improving the efficiency of charge carriers collection. The LBNL chip has been produced in the OKI $0.15 \mu \mathrm{m}$ fully depleted SOI process, made available through a collaboration with KEK, Tsukuba, Japan. The chip has arrays of both analog and binary $10 \times 10 \mu \mathrm{m}^{2}$ pixels. Their response has been characterised both in the lab and on the beam. Both the analog and the digital pixel arrays detect the high energy electrons from the ALS beam. The LBNL chip is the first SOI pixel structure to detect ionising particles on a beam. These results are very encouraging for the further development of monolithic pixel sensors in SOI technology. SOI pixel are of great interest for a broad range of applications, from beam monitoring to imaging and light source experiments, requiring fast detectors with significant on-chip data processing.

Finally, a full array of thin monolithic CMOS pixels, consisting of four planes of $50 \mu$-thin sensors for a total of over $1 \mathrm{M}$ channels has been designed, assembled and deployed on beam lines at the LBNL ALS and FNAL MBTF. This project used sensors back-thinned as part of the assessment of back-thinning yields and effects on CMOS sensors, performed as part of this LDRD. This is the first beam telescope made of thin CMOS pixel sensors. It has been extensively used to study tracking and vertexing capabilities of a prototype vertex tracker demonstrating the performances required at the ILC in terms of extrapolation resolution over the full particle momentum range. It operated in the T966 beam test experiment, carried out in collaboration with Purdue U. and INFN Padova at FNAL in summer 2007. T966 will continue data taking in 2008 and the beam telescope will become a user facility with a group from UC Santa Cruz as first user. 


\section{Effective Theories for Collider Physics}

Principal Investigator: Christian Bauer

\section{Project Description:}

In order to cleanly separate the physics we hope to discover at the large hadron collider, expected to start operation at the end of next year, from the interactions we already understand, we need a detailed understanding of the expected standard model processes. In the past, these standard model processes were studied using traditional perturbative methods, and while significant progress has been made, the difficulty has always been to deal with the complicated structure of QCD. Over the past decade, significant progress has been made to understand QCD using the framework of effective field theories (EFTs). EFTs provide a systematic way of analyzing the underlying theory in particular limits of the theory. Recently, a new effective theory called soft-collinear effective theory (SCET) has been developed by myself and collaborators, and it has proven to be tremendously successful to understand QCD for highly energetic particles. The goal of this project is to apply SCET to understand the standard model backgrounds at the LHC, which most of the time contain highly energetic particles in the initial and final states. Using the tools of effective theories should allow for a clean understanding of the required processes, with the possibility of systematic improving the description as the need arises.

\section{Accomplishments:}

Last year we have shown how SCET reproduces a commonly used approximation to QCD, called a parton shower. Parton showers are heavily used in high energy physics to theoretically generate events which can then be compared with data obtained at collider experiments. The relation between parton showers and SCET has provided us with a deeper understanding of the applicability of parton showers, and given rise for tools to go beyond the parton shower approximation. Since then we have embarked on a mission to exploit this new insight, and much of that work is now starting to bear fruit. One of the main requirements is to have an algorithmic tool, called an event generator, which is generic enough to allow the implementation of the coming SCET results. We are just finishing the first release of such a tool, which relies heavily on our new understanding of parton showers. The main feature of this algorithm is that it cleanly separates for the first time the required theoretical calculations from the algorithmic side of the event generator. As such, it can be used to implement almost any theoretical calculation possible, and in particular all SCET results to be expected.

We have also made progress in understanding how to describe hadronic initial states in the framework of the effective theory. Several subtleties needed to be addressed, which range from understanding how to factorize the non-perturbative information from the initial protons, to dealing with the necessity of so-called jet definitions in the hadronic environment. 
New Directions for Theoretical Physics at the TeV-Scale Principal Investigator: Hitoshi Murayama

\section{Project Description}

The overall motivation for this project is to address new directions in theoretical physics that brings together somewhat disparate yet connected subfields, to construct fully consistent theoretical frameworks of TeV-scale physics

Particle physicists have known since the time of Fermi (1933) that the TeV scale is an important energy scale in physics. Together with the energy scale of gravity, derived from Newton's constant, it is now viewed as one of the two fundamental energy scales of nature. Much of the development in particle physics has revolved around how we understand these two energy scales. At this time the theoretical understanding of the TeV scale is extremely poor. The only phenomenologically viable model at hand is the toy model called the Minimal Standard Model, which is obviously unsatisfactory because of its many theoretical shortcomings. Many speculations have been discussed; including such ideas as supersymmetry, large extra dimensions, warped extra dimension(s) and new strong interactions. While all of these ideas have strong theoretical appeal, and some have interesting connections to string theory, arguably the theory of everything, it is fair to say that none of them are free from problems, and their consequences have not been worked out in complete detail. Our goal then is to come up with a consistent theoretical framework that will be confronted with the anticipated beautiful data at the TeV scale from the Tevatron and the LHC

\section{Accomplishments}

Our goal can only be approached in a multi-faceted fashion, which brings together somewhat disparate yet connected subfields. To accomplish this, in FY2007 specific problems were addressed by different postdocs and students, Ben Freivogel, Eric Gimon and Joan Simon. Ben Freivogel has worked with Mina Aganagic, Christopher Beem to analyse Geometric Metastability, Quivers and Holography and with collaborators at Stanford and UC, Santa Barbara to publish a paper on collisions with a crunching bubble. He also continued his collaboration with Raphael Bousso to look into the paradox in the global description of the multiverse. Eric Gimon has worked with Thomas Levi at CCPP in New York on black ring deconstruction and the geometry of non-supersymmetric three-charge bound states. He collaborated with Petr Horava on Astrophysical violations of the Kerr bound as a possible signature of string theory. Joan Simon worked with theorists at University of Pennsylvania and others to publish an analysis of the entropy of near-external black holes in $\operatorname{AdS}(5)$ and quantum geometry and gravitational entropy. They also completed a paper on typicality versus thermality. These diverse papers help shed light on the nature of the TeV scale. 
Baryon Oscillations and Dark Energy: Prototyping Instruments

Principal Investigators: David Schlegel, Gerson Goldhaber, Eric Linder, Nikhil

Padmanabhan, Saul Perlmutter, Natalie Roe, Anthony Spadafora

Project Description

Future baryon acoustic oscillation (BAO) experiments for measuring dark energy witll require technologies to control more than 1000 fibers on the focal plane of a telescope. A simple, cost-effective approach is to individually actuate each fiber in the focal plane. This removes the complication and expense of drilling plug plates (like for the Sloan Digital Sky Survey) or building robots to move fibers meter distances with the required $30 \mu \mathrm{m}$ tolerance.

\section{Accomplishments}

Several designs for individually-actuated fibers were explored at the conceptual level. The chosen design for the LBNL fiber actuator is shown in the left figure. The design confines each fiber to move within a $2.9 \mathrm{~cm}$ diameter footprint using two motors that drive a radial $(r)$ stage and a rotational $(\theta)$ stage. All of the motors and control electronics are confined to the cylindrical space behind this footprint, such that one can close pack 1000 actuators in the SDSS focal plane. This design should achieve the requisite positional accuracy of $30 \mu \mathrm{m}$.

An initial prototype has been partly assembled, with motor controls possible on one of the two axes (right figure). The rotational stage has been completed, and tests run on the crossbar limit switch for that axis. The stepper motors are driven by identical driver/controller PC board modules. A special control scheme assures that when motors are switched off, they are at a magnetic pole position to prevent them from jumping to a nearby pole and losing position. The positioning accuracy is not yet tested.

A study of the survey strategy for the LBNL fiber positioners has been completed, and included in the SIDE spectrograph proposal for the 10.4-m Gran Telescopio Canarias. This addresses the targetting efficiencies and completeness as compared to traditional fiber positioners.

The Baryon Oscillation Spectroscopic Survey (BOSS) is one experiment that may use these actuators. For that experiment, we have received R\&D funding in August 2007 from the DOE call "Discovering the Nature of Dark Energy."
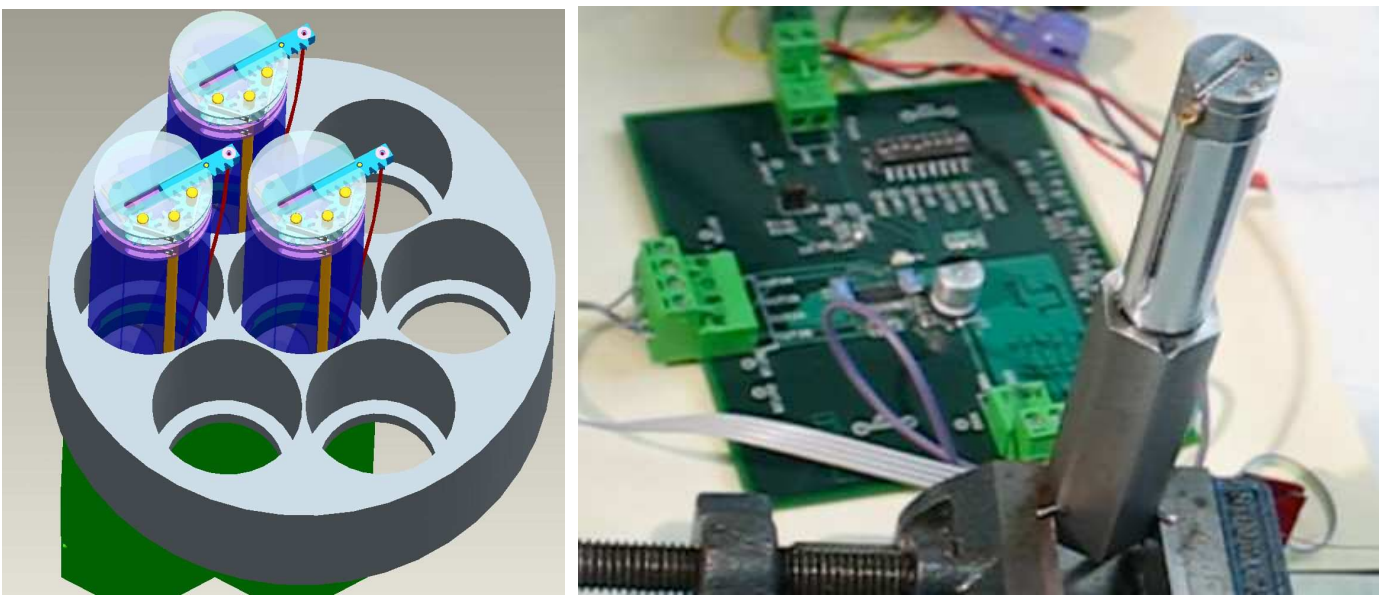

Figure 1: Conceptual drawing (left) and prototype (right) of LBNL fiber actuator. 


\section{Tests of an Advanced Neutron Detector Concept for Nuclear Fuels Monitoring Principal Investigator: Helmuth Spieler}

\section{Project Description}

The development of new reactor technologies and fuel processing is tightly coupled with the development of advanced monitoring systems. The monitoring of fresh and spent fuel at reactors and safeguards for transmutation fuels or fuel recycling will require an ensemble of monitoring technologies to optimize the measured data for the specific application. In some systems neutrons and gammas can be separated by pulse-shape discrimination, but gamma rejection is imperfect with typical leakage in the percent range. This limits the neutron detection threshold, so new neutron detector systems with improved gamma rejection would extend the sensitivity of the neutron measurement. The principle of the proposed detector is shown below.

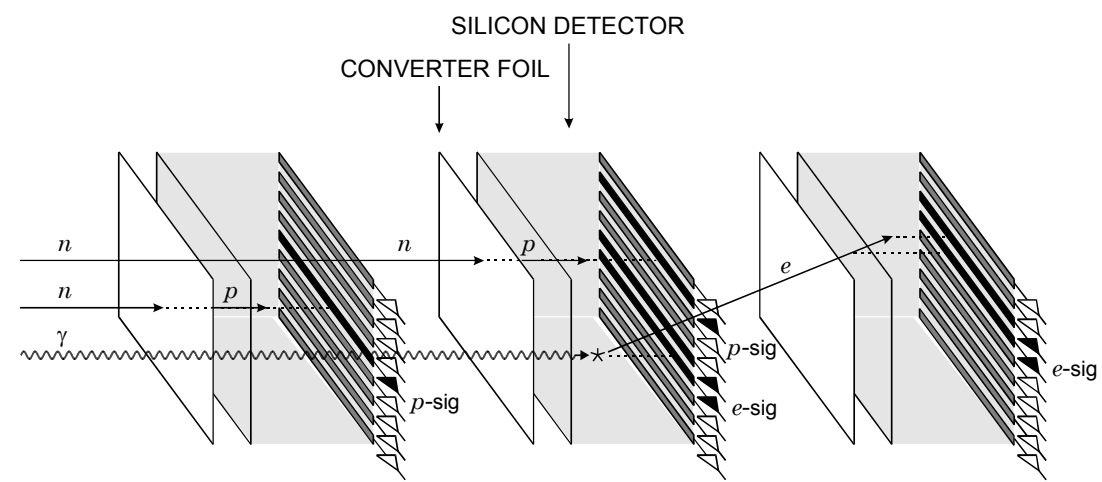

Neutrons impinging on conversion foils on the front surface of strip detectors scatter and eject recoil protons that are detected in the detector. The thickness of the silicon detector is chosen to stop the recoil protons, yielding a measurement of the recoil energy. The detectors are thin with a low detection efficiency for gamma radiation, but the gamma flux is typically several orders of magnitude higher than for neutrons, so additional techniques are needed to tag gamma hits. In active interrogation systems, time-of-flight can be utilized. The thin detectors provide fast signals, so ns time resolution is feasible. At the gamma energies of interest Compton scattering dominates, so in addition, downstream detectors are used to detect and track the Compton electrons. This requires low noise levels, achieved by utilizing a strip geometry, which also provides tracking information. This scheme exploits high-density integration techniques of sensors and electronics developed for high-energy physics together with the computing power of modern FPGAs.

The goal of the LDRD project was to measure the practical efficiency of our hybrid detectors in a mixed neutron-gamma flux. This information is essential in simulating a full system to provide the basis for a proposal to the relevant funding agency.

\section{Accomplishments}

This LDRD was only funded for the last three months of the fiscal year, so its scope did not include the full system. Commercially available single-electrode detectors were procured and assembled into basic units formed of the conversion foil and the detector. Fast lownoise preamplifiers from previous work were used. In collaboration with B. Blackburn's group at INL we took data in a beam test at the Idaho Accelerator Center in Pocatello. Detailed simulations were performed to predict the overall performance. This was especially important, as the available detectors imposed air gap between the conversion foil and the detector, which significantly degrades the recoil spectrum. The experimental data were in good agreement with simulations and through a combination of time-of-flight and energy discrimination we achieved good separation of neutrons from the gamma background with the expected efficiency. 


\section{Cross-Divisional}

Fabrication of photovoltaic devices using genetically engineered biomaterials Principal Investigator(s): Seung-Wuk Lee

\section{Project Description}

The purpose of this project is to fabricate virus-based photovoltaic devices to harness novel and clean solar energy. The monodisperse photovoltaic semiconductor nanowires will be synthesized through a mineralization process on genetically engineered viruses. Photovoltaic devices will be fabricated using semiconductor covered-virus nanowires through a conventional organic-inorganic hetero junction photovoltaic solar cell fabrication process. The approach will exploit combinatorial phage display to identify specific binding peptide information against photovoltaic semiconductor materials $\left(\mathrm{CdTe}, \mathrm{ZnO}, \mathrm{TiO}_{2}\right.$, etc), which will direct mineralization of such materials in mild aqueous conditions. Using this tactic, we will engineer monodisperse viral particles to template photovoltaic semiconducting materials. These viruses will then be vertically self-assembled on transparent electrodes to fabricate solar cell devices.

We will develop virus-based organic-inorganic hetero junction photovoltaic devices, by exploiting viruses engineered to have specific affinity between coat protein and photovoltaic semiconducting nanomaterials. The biological approach to build the proposed photovoltaic solar cell materials and devices has many promising aspects: 1) we can isolate previously unexplored novel peptide information against photovoltaic semiconducting material. Using the identified peptide information, we can design novel organisms, microbes, and biomaterials, with which we can open new fields of synthetic biology and couple many conventional micro-fabrication approaches (such as, soft lithography, DPN lithography, microprinting, and microfluidics) to build novel devices through bottom up processes. 2) Using recombinant DNA techniques, we can tune the length of the virus and subsequent nanowire scales which is suitable for the optimized device fabrication. In addition, cloning nature of the biological materials can simplify preparation of the sample through a self-replicating amplification processes. 3) Due to the diamagnetic property of the virus, we can align them vertically between the electrodes using external magnetic filed in the hetero junction devices, which is expected to enhance the efficiency of the photovoltaic devices by improving electron mobility. 4) Obtaining knowledge between biomolecules and electronic materials is critical for the design of novel nanoscale bioelectronic devices. 5) This multifaceted research project will provide graduate and undergraduate students with many opportunities to explore, integrate and educate the next generation of scientists in a variety of diverse research disciplines.

\section{Accomplishments}

We successfully constructed coat protein engineered viral library. The constructed library showed that the peptide library was successfully constructed with a little bias for certain amino acid residues. We will identify the functional virus, which can mineralize the photovoltaic semiconductors. In addition, we nucleated $\mathrm{ZnS}$ nanocrystals on viruses engineered with a specific short amino acid sequence (Fig. 1). Currently, we are optimizing the mineralization process to produce the high quality of $\mathrm{ZnS}$ nanocrystals on virus. We ultimately will use the nanowires to construct the photovoltaic cells.

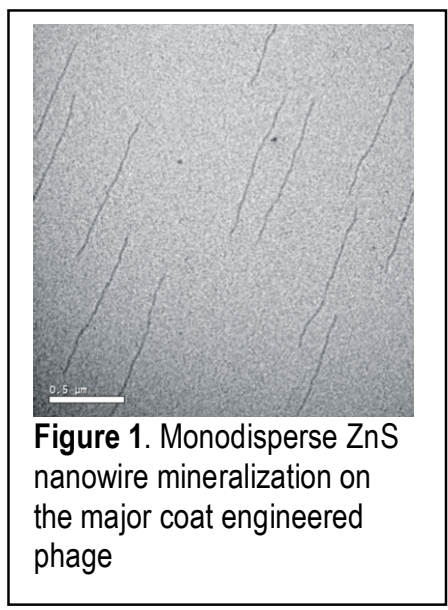




\title{
Development and Application of Quantum Monte Carlo (QMC) Methods to Biological Systems
}

\author{
Principal Investigators: William A. Lester, Jr., Graham Fleming, Michael Frenklach
}

\section{Project Description}

The purpose of this research is develop algorithmic improvements to facilitate the calculation of electronic excitations in biological and other large molecular systems using the quantum Monte Carlo (QMC) method. The highest priority is the application of these theoretical and computational developments to the study of singlet-excited states and their role in energy transfer in photosynthetic systems.

This project represents a major step forward in size system that can be treated by a rigorous quantum mechanical method for which no empirical approximations are introduced. The completion of this project will confirm the high accuracy of QMC and low-order dependence on system size, which is an impediment for the use of any other method of comparable high accuracy The methodologies developed during this LDRD will also enable new computational capabilities in the areas of nanotechnology, catalysis, and combustion, in addition to bioscience.

\section{Accomplishments}

Two significant accomplishments have been achieved. The first is the calculation of the energy transfer rate for a model system in preparation for the corresponding calculation for the photosynthetic target system. The complexity of the system and the size of the molecules targeted dictated this strategy. The next steps are the calculations on the photosynthetic systems.

The second major accomplishment has been the theoretical development and implementation in operable computer code of a QM/MM (quantum mechanics/molecular mechanics) formalism that uses QMC for the QM component and traditional approaches for the MM remainder. The approach has been successfully demonstrated on two target systems. This is a major breakthrough owing to the high accuracy of QMC for treating the reaction center and a less computationally intensive MM approach for the remainder of the system without noticeable degradation in accuracy. This development will make possible the application of the QM/MM approach to a wide range of systems of fundamental and practical interest. 



\section{Publications List}

AFRD-Fawley et al LB07015 FEL Concepts for Multiple Independent X-ray Beamlines

W.M. Fawley; "Production of Ultrashort FEL XUV Pulses via a Reverse Undulator Taper," submitted to Nuclear Instruments and Methods A, special conference issue devoted to Frontiers in FEL Physics and Related Topics, Elba Island-La Biodola, Italy; 8 - 14 September 2007; also LBNL-63558.

M. Gullans, G. Penn, and A.A. Zholents; "Performance study of a soft X-ray harmonic generation FEL seeded with an EUV laser pulse," Optics Communications 274, 167-175 (2007); also LBNL-61592.

A. Zholents and M. Zolotorev; "Attosecond x-ray pulses produced by ultra short transverse slicing via laser electron beam interaction," accepted for publication in New Journal of Physics; also CBP Tech Note-372.

G. Penn, A. Sessler, J. Wurtele; "A plasma channel beam conditioner for free electron lasers," Proceedings of the 22nd Particle Accelerator Conference (PAC07), Albuquerque, NM, 2007; also LBNL-63600. url: http://accelconf.web.cern.ch/AccelConf/p07/PAPERS/TUPMN113.PDF

J. Corlett, J. Byrd, W. M. Fawley, et al; "A high repetition rate VUV-soft x-ray FEL concept," Proceedings of the 22nd Particle Accelerator Conference (PAC07), Albuquerque, NM, 2007; also LBNL-63096. url: http://accelconf.web.cern.ch/AccelConf/p07/PAPERS/TUPMN109.PDF

AFRD-Ji LB07017 Ultra-compact Field Desorption Neutron Source for Cancer Research

Y. Wu, Q. Ji, J. Kwan, K.-N. Leung; "Recent Developments of Compact Neutron Generators at LBNL," abstract accepted and draft in preparation Joint International Workshop: Neuclear Technology and Society - Needs for next Generation, Berkeley, California, January 6-8, 2008.

Y. Wu, Q. Ji, L. Ji; "Ultra-compact Field Desorption Neutron Source for Cancer Research," in preparation for submission to CARRI 2008 (Conference on the Application of Accelerators in Research and Industry).

AFRD-Logan LB05022 Enabling High Energy Density Physics at LBNL

J.J. Barnard; J. Armijo R.M. More et al; "Theory and simulation of warm dense matter targets," Nuclear Instruments and Methods in Physics Research A 577, 275 (2007).

J.J. Barnard, N.A. Tahir, R.M. More et al; "Ion-driven warm dense matter target studies," 48th Annual Meeting of the Division of Plasma Physics, DPP06, Philadelphia, Pennsylvania, October 30-November 3, 2006

J.J. Barnard, A. Friedman, M. Marinak et al; "Simulations of ion beam heated targets for warm dense matter (WDM) physics and inertial fusion energy," 49th Annual Meeting of the Division of Plasma Physics, DPP06, Orlando, Florida, November 12-16, 2007.

K. Nakamura, B. Nagler, Cs. Toth et al; " $\mathrm{GeV}$ electron beams from $\mathrm{cm}$-scale channel guided laser wakefield accelerator," Phys. Plasmas 14, 056708 (2007).

E. Esarey, C.B. Schroeder, E. Michel, B.A. Shadwick, C.G.R. Geddes, and W.P. Leemans; "Thermal effects in plasma-based accelerators," Phys. Plasmas 14, 056707 (2007).

D. Dimitrov, R.E. Giacone, D.L. Bruhwiler et al; "Coupling of laser energy into plasma channels," Phys. Plasmas 14, 043105 (2007). 
J. van Tilborg, C. B. Schroeder, Cs. Toth, C. G. R. Geddes, E. Esarey, and W. P. Leemans; "Single-shot spatio-temporal measurements of high-field terahertz pulses," Opt. Lett. 32 (3), 313-315 (2007).

AFRD-Schroeder LB07026 Laser-plasma Accelerator Driven Free-Electron Laser with Highharmonic Seeding

C. Schroeder, W. Fawley, A. Montgomery, K. Robinson, F. Gruener, M. Bakeman, W. Leemans; "Design of a free-electron laser driven by the LBNL laser-plasma-accelerator," Nuclear Instruments and Methods in Physics Research Section A (in press); LBNL63488.

A. Montgomery, "Studies of a Free Electron Laser Driven by a Laser-Plasma Accelerator," submitted to DOE Journal of Undergraduate Research.

C. Schroeder, E. Esarey, E. Cormier-Michel, W. Leemans; "Ultra-high-order harmonic generation in cavitated plasmas," submitted to Optics Letters.

AFRD-Zholents et al LB07034 Emittance Manipulation and Beam Conditioning for FELs

Publications:

J. Qiang, I.V. Pogorelov, R.D. Ryne; "Parallel Beam Dynamics Simulation Tools for Future Light Source Linac Modeling," Proceedings of Part. Acc. Conf. PAC07, June 25-29, Albuquerque, 3522 (2007).

J. Qiang, J. Corlett, S.M. Lidia, H.A. Padmore et al; "Numerical Study of Coulomb Scattering Effects on Electron Beam from a Nano-Tip," Proceedings of Part. Acc. Conf. PAC07, June 25-29, Albuquerque, 1185 (2007).

I.V. Pogorelov, J. Qiang, R.D. Ryne, M. Venturini, A. Zholents, R. Warnock; "Simulation of the Microbunching Instability in Beam Delivery Systems for Free Electron Lasers," Proceedings Part. Acc. Conf. PAC07, June 25-29, Albuquerque, 1179 (2007).

M. Venturini and A. Zholents, "Microbunching instability from shot noise using Vlasov Solvers," Proceedings of Frontiers in FEL Physics and Related Topics Workshop, Sept. 8-14, 2007, Isola d'Elba (Italy), to appear in NIM-A (2007).

M. Venturini, "Microbunching Instability in Single-Pass Systems Using a Direct 2D Vlasov Solver," Phys. Rev. ST Accel. Beams 10, 104401 (2007), LBNL-63109.

M. Venturini, "Models of Longitudinal Space-Charge Impedance for the Study of the Microbunching Instability," submitted for publication LBNL-63108 (2007).

M. Venturini, "An Effective Longitudinal Space-Charge Impedance Model for Beams with Non-uniform and Non-axissymmetric Transverse Density," technical report, LBNL63111 (2007).

M. Cornacchia, P. Craievich, S. Di Mitri, G. Penco, M. Venturini, S. Zholents; "Running FERMI with one-stage compressor: advantages, layout, performance," technical report, LBNL-62765 (2007).

M. Venturini, R. Warnock, A. Zholents; "A Vlasov Solver for Longitudinal Dynamics in Beam Delivery Systems for x-ray FELs," Phys. Rev. ST Accel. Beams 10, 054403(2007), LBNL-62630.

J. Corlett, W. Fawley, S. Lidia et al; "FEL Design Studies at LBNL: Activities and Plans," ICFA Beam Dynamics newsletter, April 2007, 51, http://www-bd.fnal.gov/icfabd/Newsletter42.pdf. 
J. W. Staples, K. M. Baptiste, J. N. Corlett et al; "Design of a vhf-band rf photoinjector with megahertz beam repetition rate," Proceedings of Part. Acc. Conf. PAC07, June 25-29, Albuquerque, 2990 (2007), http://accelconf.web.cern.ch/AccelConf/p07/PAPERS/THPMN117.PDF.

J. Corlett, J. Byrd, W. M. Fawley et al; "A high repetition rate VUV-soft x-ray FEL concept," Proceedings of Part. Acc. Conf. PAC07, June 25-29, Albuquerque, 1167 (2007), http://accelconf.web.cern.ch/AccelConf/p07/PAPERS/TUPMN109.PDF.

\section{Presentations:}

Ji Qiang, invited talk at Workshop on Microbunching Instability, Trieste Sept. 17-19, 2007.

M. Venturini, invited talk at Frontiers in FEL Physics and Related Topics Workshop, Sept. 814 2007, Isola d'Elba (Italy).

M. Venturini, invited talk at Workshop on Microbunching Instability, Trieste Sept. 17-19, 2007.

A. Zholents, invited talk at 37th Winter Colloquium on The Physics of Quantum Electronics, Snowbird, January 2-6, 2007.

\section{AFRD-Zolotorev LB05039 Low Energy Spread Electron Source}

M. Zolotorev, E. D. Commins, F. Sannibale; "A Propsal for a Quantum-Degenerate Electron Source," Physical Review Letters 98, 184801 (2007). Also selected for the June 2007 issue of Virtual Journal of Ultrafast Science of APS.

M. Zolotorev, E. D. Commins, S. Heifets, F.Sannibale; "Proposal for a High-Brightness Pulsed Electron Source," Proceedings of the workshop on The Physics and Applications of High Brightness Electron Beams," Erice, Italy, October 2005. LBNL-60066. Now published by World Scientific in the Science and Culture Series-Physics: http://www.worldscibooks.com/physics/6621.html

E. D. Commins, "Quantum-limited electron source," Advanced Light Source Special Seminar at the LBNL, October 25, 2007.

ALS-Guo LB07016 Building In-Situ Electronic Structure Study Capability with Photon-in/ Photon-out Soft X-ray Spectroscopy

J.-H. Guo, T. Tong, L. Svec, J. Go, C. Dong, J.-W. Chiou; "Soft-x-ray Spectroscopy Experiment of Liquids," JVST A 25(4), 1231-1233 (July/August 2007).

P.-A. Glans, J.-H. Guo; "In-situ electronic structure study of $\mathrm{H}_{2}$ adsorption on HOPG," talk at APS March Meeting, Denver (March 2007).

J. Zhong, S. Barcelo, J.-W.Chiou et al; "Hydrogen Physisorption Properties of Single-Walled Carbon Nanotubes Studied by Soft-X-Ray Spectroscopy," talk at APS March Meeting, Denver (March 2007).

J.-H. Guo, "Hydrogen Adsorption on Single-Walled Carbon Nanotubes Studied by Soft-XRay Spectroscopy", invited talk Jinghua Guo, Study of Matter at Extreme Conditions, Miami Beach, Florida (April 15-20, 2007)

J. Guo, "In-situ Electronic Structure Study Capability with Photon-in/Photon-out Soft-X-Ray Spectroscopy," The 15th International Conference on Vacuum Ultraviolet Radiation Physics, Berlin, Germany (July 29 - August 3, 2007). 
ALS-Martin LB05024 Left-Handed Nanoscale Meta-Materials: Towards the Optical Domain

Z. Hao, M.C. Martin, B. Harteneck, S. Cabrini, E.H. Anderson; "Negative index of refraction observed in a single layer of closed ring magnetic dipole resonators," Appl. Phys. Lett. 91, 253119 (2007), LBNL-63710

Z. Hao, M.C. Martin, B. Harteneck, S. Cabrini, E.H. Anderson; "Double Negative Index of Refraction in a Closed Ring Metamaterial," in preparation to be submitted to Phys. Rev. Lett.

ALS-Yashchuk LB07033 Ultra-high Resolution Optics for Soft X-ray Inelastic Scattering

D. Voronov, R. Cambie, R. Feshchenko et al; "High resolution sliced multilayer grating for soft x-rays," submitted to 9th International Conference on the Physics of X-Ray Multilayer Structures (Montana, February 3-7, 2008).

D. L. Voronov, R. Cambie, R. M. Feshchenko et al; "Development of an ultra-high resolution diffraction grating for soft X-rays," Optics and Photonics 2007: Optical Engineering and Applications Symposium. OP322: Advances in X-ray/EUV Optics and Components (San Diego, August 26-30, 2007), Proc. of SPIE 6705, 67050E-1-12 (2007) - LBNL-62515.

H. Padmore, "Study of a blazed multilayer grating working in the 20th order at $1.3 \mathrm{~nm}$ wavelength," ALS Light Source Notes: LSBL-838, 5/1/2007.

D. Voronov, V. Yashchuk; "Simulation of a Sc/Si coated echelette grating with GSolver," ALS Light Source Notes: LSBL-839, 4/30/2007.

H. Padmore; "Diffraction efficiency of blazed soft x-ray multilayer gratings using scalar diffraction theory," ALS Light Source Notes: LSBL-828, 4/11/2007.

H. Padmore; "Diffraction Efficiency of Blazed Gratings in the VUV and Soft X-ray Regions," ALS Light Source Notes: LSBL-822, 4/11/2007.

D. Voronov, V. Yashchuk, H. Padmore; "Use of multilayer coated echelette in high order. Part 1: Multilayer design," ALS Light Source Notes: LSBL-820, 3/20/2007.

R. Feshchenko, D. Voronov, V. Yashchuk; "Estimation of effect of groove's periodicity distortion on performance of a diffraction grating. Part 2: Asymmetrically cut multilayer grating with normally distributed layer thicknesses," ALS Light Source Notes: LSBL-817, 2/15/2007.

R. Feshchenko, D. Voronov, V. Yashchuk; "Estimation of effect of groove's periodicity distortion on performance of a diffraction grating. Part 1: Grating with grooves normally distributed around their ideal positions," ALS Light Source Notes: LSBL-816, 2/12/2007.

V. Yashchuk, D. Voronov, R. Cambie, E. Gullikson, H. Padmore; "Ultra high resolution optics for soft x-ray inelastic scattering," ALS Light Source Notes: LSBL- 814, 2/8/2007.

H. Padmore; "Use of multilayer gratings in high order," ALS Light Source Notes: LSBL-813, $1 / 18 / 2007$.

H. Padmore; "Dispersion from an asymmetric crystal surface," ALS Light Source Notes: LSBL-812, 1/31/2007.

H. Padmore; "Simulation of multilayer grating diffraction efficiency," ALS Light Source Notes: LSBL-809, 1/8/2007.

H. Padmore; "Comparison of diffraction efficiency calculations for simple laminar soft x-ray gratings," ALS Light Source Notes: LSBL-808, 1/3/2007. 
CH-Bergman LB07006 Conversion of Glycerol and Aromatic Compounds from Biomass to Major 3- and 6- Carbon Industrial Organic Compounds

E. Arceo Rebollo, R. Bergman, J. Ellman; "An efficient method for the conversion of glycerol to allyl alcohol and evidence for the mechanism of glycerol deoxygenation," paper in preparation.

Provisional patent disclosure, application no. 60/866,661, filed January 26, 2007.

CH-Bluhm/Wilson LB07009 Chemical Reactions at Liquid/Vapor Interfaces Probed by Photoemission Spectroscopy

D.E. Starr, E.K. Wong. D.R. Worsnop, K.R. Wilson, H. Bluhm; “A combined droplet train and ambient pressure photoemission spectroscometer for the investigation of liquid/vapor interfaces," to be submitted to Chem. Phys. Lett.

CH-Prausnitz LB06027 Properties of New Ionic Liquids for Electrochemical Applications and for Extraction of Heavy-Metal Cations from Wastewaters

N, Papaïconomou, J. Salminen, J-M. Lee, and J. Prausnitz; "Physicochemical Properties of Hydrophobic Ionic Liquids Containing 1-Octylpyridinium, 1-Octyl-2-methylpyridinium or 1-Octyl-4-methylpyridinium Cations," Journal of Chemical Engineering Data, 52, 833-840 (2007), LBNL 62741.

J. Salminen, N. Papaïconomou, R. Kumar, J-M. Lee, J. Kerr, J. Newman, J. Prausnitz;

"Physicochemical Properties and Toxicities of Hydrophobic Piperidnium and Pyrrolidinium Ionic Liquids," Fluid Phase Equilibria, 261, $421-426$ (2007).

N. Papaïconomou, J-M. Lee, J. Salminen, M. Von Stosch, J. Prausnitz; "Selective Extraction of Copper, Mercury, Silver and Palladium Ions from Water Using Hydrophobic Ionic Liquids," Industrial \& Engineering Chemistry Research, O’Connell special issue forthcoming LBNL\#62914.

R. Kumar, J-M. Lee, N. Papaïconomou, J. Salminen, D. Clark, J. Prausnitz; "In vitro Cytotoxicities of Ionic Liquids: Effect of Cation Rings, Anions, and Functional Groups," submitted to Green Chemistry.

J-M. Lee, S. Ruckes, J. Prausnitz; "Solvent Polarities and Kamlet-Taft Parameters for Ionic Liquids Containing a Pyridinium Cation," submitted to Journal of Physical Chemistry B.

CH-Tilley LB05037 New Approach for the Catalytic Conversion of Methane and Other Inert Hydrocarbons

P. Bazinet, T. D. Tilley; "Octa- and Nonamethylfluorenyl Complexes of $\mathrm{Zr}(\mathrm{II}), \mathrm{Zr}(\mathrm{IV})$, and $\mathrm{Hf}(\mathrm{IV})$; Investigation of Steric and Electronic Effects," accepted for publication in Organometallics.

CS-Bell LB07005 Structured, Adaptive Mesh Refinement Method for Multiphase Reactive Transport in Groundwater

A. Almgren, J. Bell, G. Pau, M. Lijewski; “Adaptive Mesh Refinement for Incompressible Flow in Porous Media," in preparation for submission to J. Comp. Phys. 
J. Bell, D. E.A. van Odyck, F. Monmont, N. Nikiforakis; "The Mathematical Structure of Multiphase Thermal Models of Flow in Porous Media," in preparation for submission to Comm. Appl. Math. \& Comp. Sci.

CS-Berket/Agarwal LB07007 On-demand Overlays for Scientific Applications

K. Berket, C. Guok, J. Lee; "Improving the Bulk Data Transfer Experience," submitted to special issue of IJIPT Journal on Management of IP Networks and Services.

CS-Chevassut LB05008 Cryptographic Foundations for New Generation Distributed Systems

O. Chevassut, J. Milner, D. Pointcheval; "Security Proof for Password Authentication in TLS - Verifier-based Three-Party Group Diffie-Hellman," to appear in 2008, LBNL-59994.

M. Abdalla, O. Chevassut, B. Moeller, D. Pointcheval: "Strong Password-Based Authentication in TLS Using the Three-Party Group Diffie-Hellman Protocol," International Journal of Security and Networks (IJSN, 2007). LBNL-59947.

CS-Markowitz LB06015 Integrated Microbial Community Genomes Data Management System

Metagenome Data Management and Analysis Tools

V.M. Markowitz, N.N. Ivanova, E. Szeto et al; "IMG/M: a data management and analysis system for metagenomes." Nucleic Acids Research 38, Database Issue.

D. Dalevi, V.M. Markowitz, E. Szeto et al; "Confidence estimates for gene-centric analyses of metagenomic data," submitted to BMC Bioinformatics.

D. Dalevi, N.N. Ivanova, K. Mavromatis et al; "Annotation of Metagenome Short Reads Using Proxyegenes," submitted to EECB'08 European Conf. on Comp. Biology.

Metagenome Studies using Analysis Tools developed as part of the LDRD:

Y. Marcy, C. Ouverney, E.M. Bik et al; "Dissecting biological dark matter: single cell genetic analysis of TM7, a rare and uncultivated microbe from the human mouth," Proceedings of the National Academy of Sciences 104:11889-11894.

V. Kunin, S. He, F. Warnecke et al; "A bacterial metapopulation adapts locally to phage predation despite global dispersal," submitted for publication in PLoS Biology.

F. Warnecke, P. Luginbuhl, N. Ivanova et al; "Metagenomic and functional analysis of hindgut microbiota of a wood-feeding higher termite," Nature 450: 560 - 565.

M.G. Kalyuzhnaya, A. Lapidus, N. Ivanova et al; "High resolution metagenomics targets major functional types in complex microbial communities," to be submitted to Nature.

Presentations of the Metagenome Data Management and Analysis Tools:

—; "Metagenome Sequence Data Management and Analysis," CHI Conference on Next Generation Sequencing Applications and Case Studies, San Diego, March 2007.

—; "Metagenome Sequence Data Management and Analysis," Systems Biology Workshop: From Nucleotides to Ecosystems, Melbourne, Australia, May 2007.

—; "Metagenome Sequence Data Management and Analysis," Metagenome 2007 Conference, San Diego, July 2007.

-; "Towards a Rational Genome and Metagenome Dataspace," US-EC Workshop on Cyberinfrastructure Resources for Genome-Enabled Research on Microbial Life and the Marine Environment, Sep 2007. 
CS-Oliker LB05029 Enhancing Commodity Scalar Processors with Vector Components for Increased Scientific Productivity

S. Williams, J. Shalf, L. Oliker, P. Husbands, S. Kamil, K. Yelick; "The Potential of the Cell Processor for Scientific Computing," ACM International Conference on Computing Frontiers, May 2006. url: http://crd.lbl.gov/ oliker/papers/CF06_cell.pdf.

S. Williams, J. Shalf, L. Oliker, P. Husbands, S. Kamil, K. Yelick; "Scientific Computing Kernels on the Cell Processor," International Journal of Parallel Programming (IJPP), DOI 10.1007/s10766-007-0034-5, April 2007.

S. Williams, L. Oliker, R. Vuduc, J. Shalf, K. Yelick, J. Demmel; "Optimization of Sparse Matrix-Vector Multiplication on Emerging Multicore Platforms," SC07: International Conference for High-Performance Computing, Networking, Storage, and Analysis, Nov. 2007. url: http://crd.lbl.gov/ oliker/papers/SC07_SPMV.pdf.

S. Williams, J. Carter, L. Oliker J. Shalf, K. Yelick; "Lattice Boltzmann Simulation Optimization on Leading Multicore Platforms," submitted to International Parallel \& Distributed Processing Symposium (IPDPS) 2008.

J. Gebis, L. Oliker, J. Shalf, S. Williams, K. Yelick; "Improving Memory Subsystem Performance using ViVA: Virtual Vector Architecture," submitted to International Symposium on Computer Architecture (ISCA), 2008.

CS-Pinar et al LB05031 Advanced Computational Tools for Electric Power Systems

V. Donde, V. Lopez, B. Lesieutre, A. Pinar, C. Yang, J. Meza; "Severe Multiple Contingency Screening in Electric Power Systems," to be published in IEEE Transactions on Power Systems.

A. Pinar, J. Meza, V..Donde, B. Lesieutre; "Optimization Strategies for the Vulnerability Analysis of the Electric Power Grid," submitted to SIAM Optimization.

A. Pinar, A. Reichert, B. Lesieutre; "Computing Criticality of Lines in Power Systems," Proc. 2007 IEEE International Symposium on Circuits and Systems.

A. Pinar, Y. Fogel, and B. Lesieutre; "The Inhibiting Bisection Problem," technical report LBNL-62142.

B. Lesieutre, A. Pinar, S. Roy; "Power System Extreme Event Detection: The Vulnerability Frontier," to be published in Proc. $41^{\text {st }}$ Hawaii International Conference on System Sciences.

CS-Romano LB05032 Statistical Feature Modeling for Scientific Data Via Basis Decomposition

S. Bailey et al; "How To Find More Supernovae With Less Work," AAS 209, Winter 2007.

C. Ding, T. Li; "Adaptive Dimension Reduction Using Discriminant Analysis and K-means Clustering." Int'l Conf. on Machine Learning, 2007.

T. Li, C. Ding, M. Jordan; "Solving consensus and Constraint Clustering Problems Using Nonnegative Matrix Factorization," Int'l Conf on Data Mining, Nov 2007. 
CS-Shalf LB07028 Power Efficiency Metrics for High Performance Computing

J. Shalf, D. Bailey; "Power Efficiency and the Top500," IEEE Supercomputing 2006 Conference in Seattle, Washington, November 14, 2006. url: http://www.nersc.gov/projects/SDSA/reports/uploaded/Top500PowerEff.pdf

S. Kamil, J. Shalf; "Power Efficiency Metrics for the Top500," International Supercomputing Conference (ISC2007) in Dresden Germany, June, 2007. url: https://www.nersc.gov/projects/SDSA/reports/uploaded/ISC Top500Power.pdf

J. Shalf; "NERSC Power Efficiency," NERSC User's Group Meeting, September 17, 2007. url: http://www.nersc.gov/projects/SDSA/reports/uploaded/Shalf_NUG2006_Power.pdf

J. Shalf, S. Kamil, E. Strohmaier, D. Bailey; "Power Efficiency for HPC Applications," IEEE Supercomputing 2007 Conference, Reno Nevada, November 14, 2007. url: http://www.nersc.gov/projects/SDSA/reports/uploaded/Top500PowerNov14SC07.pdf

J. Shalf; "Power, Cooling, and Energy Efficiency for the Petascale and Beyond," IEEE Supercomputing 2007 Conference, Reno Nevada, November 15, 2007. url: https://www.nersc.gov/projects/SDSA/reports/uploaded/SC07_Power JS final.pdf (part of larger panel discussion organized by LDRD participants) http://esdc.pnl.gov/12 SC07 BOF.stm

S. Kamil, J. Shalf; "Measuring Power Efficiency for NERSCs Newest Flagship Machine," LBNL Technical Report LBNL-64259, March 2007. url: https://www.nersc.gov/projects/SDSA/reports/uploaded/powereff_report_xt4.pdf

S. Kamil, J. Shalf; "Estimating Power Efficiency for HPC Systems," accepted for publication in International Parallel and Distributed Computing (IPDPS) workshop on High Performance Power-Aware Computing (HPPAC).

CS-Skinner LB07029 Integrated Performance Monitoring of Grid and HPC Workloads

D. Skinner, "Integrated Performance Monitoring," Software Development Tools for PetaScale Computing Workshop, Aug 2, 2007. Poster at Workshop: http://www.csm.ornl.gov/workshops/Petascale07/.

CS-Wilkening LB06026 Extended First Order System Least Squares Finite Elements

J. Wilkening; " An algorithm for computing Jordan chains and inverting analytic matrix functions," Linear Algebra and its Applications 427 (2007), 6-25.

J. Wilkening, "Practical error estimates for Reynolds' lubrication approximation and its higher order corrections," submitted to SIAM Journal on Mathematical Analysis. Preprint server: arXiv:0706.4103v1, 27 Jun 2007

J. Wilkening, "Inf-sup estimates for the Stokes problem in a periodic channel," submitted to SIAM Journal on Mathematical Analysis. Preprint server: arXiv:0706.4082v1, 27 Jun 2007.

J. Wilkening, "Stable asymptotics for corner singularities of elliptic systems," to be submitted to Comm. Pure. Appl. Math.

J. Wilkening, "Extended first order system least squares finite elements," to be submitted to SIAM Journal on Numerical Analysis. 
ESD-Berryman LB07008 Applications of Adjoint Field Methods and Time-Reversal Data Processing to Inverse Problems in Electromagnetics, Seismics, and Ultrasonics

D. H. Chambers, J. G. Berryman; "Target characterization using decomposition of the timereversal operator: Electromagnetic scattering from small ellipsoids," Inverse Problems 22, 2145-2164 (2006).

ESD-Coates et al LB06004 An Investigation of the Microbial Processes Involved in Electron Transfer Onto the Anode of a Biological Fuel Cell

\section{Publications:}

K. Wrighton, P. Agbo, F. Warnecke, K.A. Weber, E. Brodie, T. DeSantis, P. Hugenholtz, G. Andersen, J.D. Coates; "Microbial ecology of electricity generating thermophilic microbial fuel cells," submitted to PNAS.

J.C. Thrash, J.D. Coates; "Direct and Indirect Electrical Stimulation of Microbial Metabolism," submitted to Environ. Sci. Technol.

\section{Abstracts and poster presentations:}

J.C. Thrash, L.A. Achenbach, J.D. Coates; "Bioelectrical Perchorate Remediation," 2007 American Geophysical Union Fall Meeting.

J.C. Thrash, L.A. Achenbach, J.D. Coates; "Continuous Bioelectrical Perchlorate Treatment," 2007 SERDP Partners in Environmental Technology Technical Symposium \& Workshop.

J.C. Thrash, L.A. Achenbach, J.D. Coates; "Mediatorless Bioelectrical Perchlorate Reduction," American Society for Microbiology $107^{\text {th }}$ General Meeting.

J.D. Coates, J.C. Thrash, L.A. Achenbach; "Bioelectrical Perchlorate Reduction," Battelle $9^{\text {th }}$ International In-Situ and On-Site Bioremediation Symposium.

J.C. Thrash, "Bioelectrical Perchlorate Reduction," UC Berkeley Microbiology Student Group $8^{\text {th }}$ Annual Intercampus Microbiology Student Symposium.

K.C. Wrighton, P. Agbo, F. Warnecke, E.L. Brodie, K.A. Weber, C. Chow, T.Z. DeSantis, G. Andersen, J.D. Coates; "The Microbial Ecology of Thermophilic Microbial Fuel Cell," American Geophysical Union Fall Meeting, San Francisco, December 2007.

K.C. Wrighton, P. Agbo, K.A. Weber, E.L. Brodie, Y. Piceno, J.C. Thrash, G. Andersen, L. Achenbach, J.D. Coates; "Electrochemical Performance and Microbial Characterization from a Thermophilic Microbial Fuel Cell," American Society of Microbiology, Toronto, Canada, May 2007.

K.C Wrighton, P. Agbo, J.D. Coates; "Electrochemical Performance from a Thermophilic Microbial Fuel Cell,” Conference on Biofuels, Berkeley, California, March 2006.

\section{Patent Submission:}

J.D. Coates, J.C. Thrash, "Bioelectrochemical treatment of xenobiotics," application number 60/975,584- patent pending.

ESD-DePaolo LB05011 Micro-characterization and Chemical Micro-dynamics of Atmospheric Mineral Dust

J.N.Christensen, S.S. Cliff, R.A. VanCuren, K.D. Perry, D.J. DePaolo; "Using Isotopes to Track Intercontinental Dust Transport: Building a Bridge from Hefei, China to 
California," Geochimica et Cosmochimica Acta, 71 (15S), A172 (2007); Spring 2007, Goldschmidt Conference Abstracts.

D.J. DePaolo, G.P. Lu; "Kinetic isotope effects in snow crystal growth: Lattice-Boltzman approach," Geochimica et Cosmochimica Acta 71 (15), A218-A218 (2007); Suppl. S Aug 2007, Goldschmidt Conference Abstracts.

G.P. Lu, D.J. DePaolo; "Lattice Boltzmann simulation of oxygen- and hydrogen-isotope fractionations during snow crystal formation," Geological Society of America Abstracts with Programs, 39 (6), 15.

G.P. Lu, D.J. DePaolo, Q. Kang, D. Zhang; "Lattice Boltzmann Simulation of Snow Crystal Growth in Clouds," in preparation for Geochimica et Cosmochimica Acta,

G.P. Lu, D.J. DePaolo; "Lattice-Boltzmann models of water isotope fractionation during snow crystal growth in clouds," in preparation for J. Geophys. Res.

J.N.Christensen, S.S. Cliff, R.A. VanCuren, K.D. Perry, D.J. DePaolo; "Using Isotopes to Track Intercontinental Dust Transport," in preparation for Geochimica et Cosmochimica Acta,

ESD-Gilbert LB05003 Behavior and Impact of Nanoparticles in the Environment

Full citations of publications listed in 2006 report:

B. Gilbert, C. S. Kim, C.-L. Dong, J. Guo, P. S. Nico, D. K. Shuh; "Oxygen K-edge emission and absorption spectroscopy of iron oxyhydroxide nanoparticles," in X-ray Absorption Fine Structure-XAFS 13. Edited by B. Hedman and P. Pianetta, American Institute of Physics Conference Proceedings, vol. 882, X-RAY ABSORPTION FINE STRUCTURE - XAFS13: 13th International Conference, Stanford, California (USA), 9-14 July 2006, p. 51-55 (2007), LBNL-62225.

B. Gilbert, G. Lu, C. S. Kim; "Stable nanoparticle clusters formed under environmentally relevant conditions," Journal of Colloid and Interface Science 313, 152-159, LBNL60208.

B. Chen, B. Gilbert, H. Zhang, J. F. Banfield; "Mechanism of nanoparticle growth and phase transformation inhibition by surface impurities," Phys. Rev. Lett. 98, 106103 (2007), LBNL\#-63060

J. Cervini-Silva, B. Gilbert, S. Fakra, S. Freidlich, J. F. Banfield; "Decarboxylation and polymerization of catechol and formation of $\mathrm{CeO}_{2}$ due to coupled redox and dissolution reactions at the surface of cerium(III) phosphate," in press Geochim. et Cosmochim. Acta.

Publications resulting from 2007 work:

G. Ren, F. Huang, B. Gilbert, J. Zhang, Z. Lin, J. Liang; "The evolution of ZnS nanosheet morphology controlled by interfacial free energy," submitted to Chemistry of Materials.

C. Goodell, B. Gilbert, S. Weigand, H. Zhang, J. F. Banfield; "The kinetics of the water adsorption driven structural transformation of $\mathrm{ZnS}$ nanoparticles," in second review $J$. Phys. Chem. C.

B. Gilbert, C. Frandsen, D. M. Sherman; "Soft x-ray spectroscopy of hematite nanoparticles," in preparation for submission to Phys. Rev. B. 
ESD-Moridis LB06016 Interrelation of Global Warming and Hydrate Dissociation in Oceanic Accumulations

M. Reagan, G. Moridis; "Oceanic Gas Hydrate Instability And Dissociation Under Climate Change Scenarios," Geophys. Res. Lett. 34, L22709 (November 27, 2007).

M. Reagan, G. Moridis; "Dynamic Response Of Oceanic Hydrate Deposits To Ocean Temperature Change," submitted to J. Geophysical Research: Oceans.

ESD-Oldenburg LB06018 Coupled Modeling of Hydrology, Nutrient Cycling, and Vegatation: Applications to Water Quality and Water Balance

C. Gu, F. Maggi, W.J. Riley et al; "Aqeuous and gaseous nitrogen losses induced by fertilizer application," submitted to J. of Geophysical Research - Biogeosciences.

C. Gu, F. Maggi, W.J. Riley, C.M. Oldenburg; "Lime and soil moisture effects on nitrogen gas loss following fertilizer application," abstract for AGU Fall Meeting. San Francisco, CA.

F. Maggi, C. Gu, W.J. Riley et al; "Mechanistic modeling of nitrogen cycling and losses," in press in J. of Geophysical Research - Biogeosciences.

F. Maggi, C. Gu., W.J. Riley, C.M. Oldenburg; "Nitrogen cycle modeling: a mechanistic estimate of N-losses from agricultural fields over the seasonal time period," abstract for AGU Fall Meeting, San Francisco, CA.

W.J. Riley, F. Maggi, C. Gu; "Mechanistic representation of the $\mathrm{N}$ isotope composition of pools and fluxes in a coupled soil and plant system: model development, testing, and application," abstract for AGU Fall Meeting, San Francisco, CA.

ESD-Steefel LB05036 Biogeochemical Reaction Rates and Pathways in Porous Media

L. Li, C.I. Steefel, L. Yang; "Scale dependence of mineral dissolution rates within single pores and fractures," in press Geochimica et Cosmochimica Acta.

L. Yang, C.I. Steefel; "Kaolinite dissolution and precipitation kinetics at $22^{\circ} \mathrm{C}$ and $\mathrm{pH}$ 4," Geochimica Cosmochimica Acta 72(1), 99-116.

C.I. Steefel, "Geochemical kinetics and transport," in Kinetics of Water-Rock Interaction (eds. S.L. Brantley, J.D. Kubicki, A.F. White), Springer, New York, pp. 545-589.

EG-Denes et al LB05010 Novel Imaging Detectors for Materials and Biology

P. Denes et al, "Active Pixel Sensors for electron microscopy," Nuclear Inst. and Methods in Physics Research A 579 (2), 891-894 (Sep 2007).

P. Denes, presentation given at Advanced Instrumentation Colloquium, SLAC, Mar. 2007.

P. Denes, "Active Pixel Sensors for Electron Microscopy," presentation given at Vertex 2007, Lake Placid, NY, Sep. 2007.

EETD-Destaillats et al LB07014 Understanding the Chemistry of Innovative Air Cleaning Technologies

D. Kibanova, M. Trejo, H. Destaillats, J. Cervini-Silva; "Synthesis of clay-TiO nanocomposites with photocatalytic activity for the degradation of air pollutants," submitted to Applied Clay Science. 
H. Destaillats, D. Kibanova, J. Cervini-Silva and A.T. Hodgson; "Surface species formed during photooxidation of adsorbed organic pollutants in air," Proceedings of the $12^{\text {th }}$ international conference on $\mathrm{TiO}_{2}$ photocatalysis $\left(\mathrm{TiO}_{2}-12\right)$, Niagara Falls, NY, September 2007.

J. Cervini-Silva, D. Kibanova, M. Trejo and H. Destaillats; "The use of small-particle size $\mathrm{TiO}_{2}$ supported on clays as photocatalytic materials: a low-cost alternative technology for the degradation of air pollutants," Proceedings of the $44^{\text {th }}$ Annual Meeting of the Clay Minerals Society, Santa Fe, NM, June 2007.

D. Kibanova, M. Trejo, H. Destaillats and J. Cervini-Silva; "The use of $\mathrm{TiO}_{2}$ supported on nanostructured materials as photocatalysts: a low-cost alternative technology for the degradation of air pollutants," Proceedings of the American Geophysical Union Joint Assembly 2007, Acapulco, Mexico, May 2007.

EETD-Gadgil LB06007 Arsenic ElectroChemistry: Exploring Electrochemical Arsenic Remediation

S. Amrose, A. Gadgil, V. Srinivasan, R. Kostecki; "Electrochemical Arsenic Remediation Using Iron for Bangladesh," in preparation for submission to Water Research.

S. Amrose, A. Gadgil, V. Srinivasan, R. Kostecki; "Mechanism of Arsenic Removal in an Electrochemical Prosess with Iron: an EXAFS Study," in preparation for submission to Chemosphere.

Posters:

S. Amrose, A. Gadgil, K. Kowolik, V. Srinivasan, R. Kostecki; "Low-cost Electrochemical Arsenic Remediation for Rural Bangladesh," poster for ESW San Francisco Conference, Feb 8-9, 2008.

EETD-Kostecki/Mao LB06013 Surface Plasmon-Enhanced Photovaotaic Device

X. Chen, S. S. Mao; "Synthesis of titanium dioxide nanomaterials," J. of Nanoscience and Nanotechnology 6, 906 (2006).

X. Chen, M. Schriver, T. Suen, S. S. Mao; "Fabrication of $10 \mathrm{~nm}$ diameter $\mathrm{TiO}_{2}$ nanotube arrays by titanium anodization," Thin Solid Films 515, 8509 (2007).

X. Chen, S. S. Mao; "Titanium dioxide nanomaterials: synthesis, properties, modifications, and applications," Chemical Reviews 107, 2891 (2007).

\section{EETD-Levine LB07036 China's Energy Future: Changes in Energy Intensity}

J. Lin, N. Zhou, M. Levine, D. Fridley; "Achieving China's target for energy intensity reduction in 2010: an exploration of recent trends and possible future scenarios," accepted for publication in Energy Policy, LBNL-61800.

EETD-McMahon et al LB07021 Integrated Decision Support Tool for Joint Optimal Control of Energy and Water Systems under Uncertainty

P. Schwartz, P. Collela; "A second order method for solving the eikonal equation," in preparation for submittal to $J$. of Appl. Math.

N. L. Miller, L. L. Dale, S. D. Vicuna, T. N. Kadir, E. C. Dogrul, C. F. Brush; "Drought Analysis of the California Central Valley Surface-Groundwater-Conveyance System," in preparation for submittal to J. of Amer. Water Resources Assoc. 
B. Faybishenko et. al; "Evaluation of the Epistemic Uncertainty of Evapotranspiration and Groundwater Recharge Using Maximum Likelihood Bayesian Averaging," in preparation for submittal to Water Resources Research.

L. Dale, B. Faybishenko, P. Schwartz, N. Miller; "Optimization of Water Energy Use in the Sacramento Valley under Different Climate Scenarios," in preparation for submittal to Land Economics.

EETD-Newman et al LB06021 Photons to Fuels - the Electrochemical Reduction of Carbon Dioxide to Methanol

C. Delacourt, P. Ridgway, J. Kerr, J. Newman; "Design of an Electrochemical Cell Making Syngas $\left(\mathrm{H}_{2}+\mathrm{CO}\right)$ from $\mathrm{CO}_{2}$ and $\mathrm{H}_{2} \mathrm{O}$ Reduction at Room Temperature," $J$. of the Electrochemical Society 155, B42-B49 (2008).

C. Delacourt, J. Newman; "Mathematical modeling of a cation-exchange membrane containing two cations," in preparation for submittal to J. of the Electrochemical Society.

B. Boonyaratanakornkit, R. Mehlhorn, R. Kostecki, D. Clark; "Whole Cell Reduction of Carbon Dioxide to Formate in an Electrochemical System," in preparation for submittal to Federation of European Biochemical Societies Letters.

GN-Eisen LB06033 Computational and Experimental Testing of Methods for Binning Sequences from Metagenomic Studies

S. Chatterji, I. Yamazaki, Z. Bai, J.A. Eisen; "CompostBin: A DNA composition-based algorithm for binning environmental shotgun reads," accepted for publication in Lecture Notes in Computer Science.

GN-Hugenholtz LB06010 Matagenomics-Enabled Analysis of Termite Hindgut Microbiota for Biomass Conversion and Cleaner Energy

F. Warnecke, P. Luginbühl, N. Ivanova et al; "Metagenomic and functional analysis of hindgut microbiota of a wood-feeding higher termite," Nature 450 (7169), 560-565.

LSD-Butland LB07010 Functional Interactomics: Integrating Physical and Functional Interaction Networks

G. Butland, M. Babụ, H. Lo et al; "eSGA: E. coli Synthetic Genetic Array analysis," in preparation for submission to Nature Biotech.

LSD-Cooper LB07013 Transcription CoFactor PC4 Interactions with RNA Polymerase and XPG in Transcription-Coupled Repair

A.H. Sarker, S. Mitra, T. Hazra, P.K. Cooper; "Interactions between NEIL2 and XPG: Implications for a Role of Transcription-Coupled Repair Proteins in Repair of Oxidative DNA Damage," on preparation for submission to Molecular Cell. 
LSD-Schild LB06022 Determining if PIR51 is a Potential Tumor Suppressor Gene Similar to BRCA2

C. Wiese, E. Dray, T. Groesser et al; "Promotion of Homologous Recombination and Genomic Stability by RAD51AP1 via RAD51 Recombinase Enhancement," Mol Cell 28, 482-90 (2007, this paper shared the cover of Molecular Cell with a second article).

LSD-Wyrobek LB06035 Expression Profiling of Radiation and Cancer Susceptibility Genes

F. Marchetti, M. Coleman, I. Jones, A. Wyrobek; "Candidate protein biomarkers of human exposure to ionizing radiation, "International Journal of Radiation Biology 82 (9), 60539 (Nov. 2006).

A. Wyrobek, F. Marchetti, X. Lowe et al; "Molecular mechanisms and cellular consequences of low-dose exposure to ionizing radiation," abstract in Annual DOE Low-Dose Workshop, January 2007.

A.J. Wyrobek, "Molecular and bioinformatics approaches to assessing individual biodosimetry and radiation susceptibility," Proceedings of the International Congress of Radiation Research, June 2007. Abstract printed in Radiation Research journal.

T.E. Schmid, M. Marchetti, S. Bhatnagar, A.J. Wyrobek; "Low-dose radioadaptive response of mouse blood and brain tissue to DNA damage," Proceedings of the International Congress of Radiation Research, June 2007. Abstract printed in Radiation Research journal.

A.J. Wyrobek, "Genomic effects of low-dose ionizing radiation and tissue risks for genetic diseases," abstract in Proceedings of the $32^{\text {nd }}$ National Congress of Human Genetics, October 2007.

T. Schmid, F. Marchetti, A. Polyzos, S. Bhatnagar, A. Wyrobek; "The Tp53 gene is a key regulator of the radioadaptive response for DNA damage in vivo," Proceeding of the $38^{\text {th }}$ National Meeting of the Environmental Mutagen Society, October 2007. Abstract printed in Environmental and Molecular Mutagenesis journal.

A.J. Wyrobek, S. Bhattacharya, F. Marchetti, C. Manohar, K. Krishnan, M. Coleman; "Genomic analyses of the gene expression pathways induced by low-dose ionizing radiation," in preparation for submission to International Journal of Radiation Biology.

A.J. Wyrobek, M. Coleman, D. Nelson, F. Pearson; "Cellular expression profiles and gene pathways vary with increasing doses of low-LET ionizing radiation," in preparation for submission to Radiation Research.

MSD-Francis et al LB06005 Tailoring the Self Assembly of Functionalized Biomolecular Building Blocks

S.W. Chung, A.D. Presley, M.B. Francis, J.J. DeYoreo; "Scanning probe-based fabrication of 3D nanostructures via affinity templates, functional RNA and meniscus-mediated surface remodeling," Scanning (in press).

MSD-Javey LB06038 Integration of Synthetic Nanomaterials for High Performance, Robust, and Flexible Circuitry

R. Yerushalmi, Z. A. Jacobson, J. C. Ho, Z. Fan, A. Javey; "Large scale, highly ordered assembly of nanowire parallel arrays by differential roll printing," Appl. Phys. Lett. 91, 203104 (2007). 
Z. Fan, J. C. Ho, Z. A. Jacobson, R. Yerushalmi, R. L. Alley, H. Razavi, A. Javey; "WaferScale Assembly of Highly Ordered Semiconductor Nanowire Arrays by Contact Printing," Nano Letters, ASAP (2007).

MSD-Kaindl LB06011 Terahertz-Frequency Conductivity and Ultrafast Optical Excitations in Single-Walled Carbon Nanotubes

R. A. Kaindl, B. A. Schmid, R. Huber, D. S. Chemla, Y.-Z. Ma, M. W. Graham, G. R. Fleming; "Ultrafast THz Dynamics in Single-Walled Carbon Nanotubes," in preparation for submission to $P R L$ or possibly Science or Nature.

J. Wang, R. A. Kaindl, N. C. Nielsen, D. S. Chemla, Y.-Z. Ma, M. W. Graham, G. R. Fleming, "Photoinduced Mid-Infrared Excitations in Single-Walled Carbon Nanotubes, in preparation for submission to $P R L$ or possibly Science or Nature.

MSD-Ritchie LB06020 Aging, Disease and the Mechanical Response of Biological Tissues, Specifically in Human Bone

J. W. Ager III, R. K. Nalla, G. Balooch, G. Kim, M. Pugach, S. Habelitz, G. W. Marshall, J. H. Kinney, R. O. Ritchie; "On the Increasing Fragility of Human Teeth with Age: A Deep-Ultraviolet Resonance Raman Study,” J. Bone Min. Res. 21, 1879-1888 (2006).

W. Yao, Z. Cheng, K. J. Koester, J. W. Ager, S. Chefo, G. Balooch, M. Balooch, A. Pham, C. Busse, R. O. Ritchie, and N. E. Lane, "The distribution and degree of bone mineral is maintained with intravenous bisphosphonates in aged estrogen deficient rats and is a strong predictor of bone strength," Bone 41, 804-812 (2007).

G. Balooch, W. Yao, J. W. Ager, M. Balooch, R. K. Nalla, A. E. Porter, R. O. Ritchie, N. E. Lane; "The aminobisphosphonate, risedronate, preserves localized mineral and material properties of bone in the presence of glucocorticoids," Arthritis and Rheumatism 56, $3726-3737$ (2007).

K. J. Koester, J. W. Ager III, R. O. Ritchie; "How really tough is human bone? Evolution of crack-growth resistance using in-situ electron microscopy," submitted to Nature Materials.

K. J. Koester, J. W. Ager III, R. O. Ritchie; "The effect of aging on crack-growth resistance and toughening mechanisms in human dentin," submitted to Biomaterials.

R. O. Ritchie, S. Ionova, K. J. Koester, N. E. Lane, J. W. Ager III; "Measurement of the toughness of bone: A tutorial with special reference to small animal studies," submitted to Bone.

MSD-Somorjai LB06023 Electron Flow Generated by Gas Phase Exothermic Catalytic Reactions Using Metal-Semiconductor Nanodiodes

J.Y. Park, J.R. Renzas, B.B. Hsu, G.A. Somorjai; "Interfacial and Chemical Properties of $\mathrm{Pt} / \mathrm{TiO}_{2}, \mathrm{Pd} / \mathrm{TiO}_{2}$ and $\mathrm{Pt} / \mathrm{GaN}$ Catalytic Nanodiodes Influencing Hot Electron Flows," J. Phys. Chem. C 111 (42), 15331 -15336 (2007).

J.Y. Park, J.R. Renzas, A.M. Contreras, G.A. Somorjai; "The Genesis and Importance of Oxide-Metal Interface Controlled Heterogeneous Catalysis; The Catalytic Nanodiode," Topics in Catalysis 46, 217-222 (2007).

G.A. Somorjai, J.Y Park; "The Impact of Surface Science on the Commercialization of Chemical Processes," Catalysis Letters 115, 87 (2007). 
G.A. Somorjai, R.L. York, D. Butcher, J.Y. Park; "The Evolution of Model Catalytic Systems; Studies of Structure, Bonding and Dynamics from Single Crystal Metal Surfaces to Nanoparticles, and from Low Pressure $\left(<10^{-3}\right.$ Torr $)$ to High Pressure $\left(>10^{-3}\right.$ Torr) to Liquid Interfaces," Phys. Chem. Chem. Phys. 9, 3500-3513 (2007, invited review paper).

G.A. Somorjai, J.Y Park; "Frontier of Surface Science," Physics Today 60, 48 (2007, feature article).

MSD-Stamper-Kurn LB07030 Studies of Quantum Antiferromagnetism in Two-dimensional Triangular Lattices Using Ultracold Atoms

M. Vengalattore, J.M. Higbie, S.R. Leslie, J. Guzman, L.E. Sadler, D.M. Stamper-Kurn; "High-Resolution Magnetometry with a Spinor Bose-Einstein Condensate," Phys. Rev. Lett. 98, 200801 (2007).

MSD-Yang et al LB07032 Hierarchically Nanostructures Systems for Solar Energy Hydrogen Production

W.H. Harman, A.L. Chang, C.J. Chang; in preparation for submission to ACS journals.

R.J. Wright, C. Lim, T.D. Tilley; "Proton reduction catalysts: Iron-only hydrogenase mimics possessing structurally rigid dithiolates," in preparation for submission to ACS journals.

Y. Huang, M. Fardy, P. Yang; "Semiconductor heterojunctions with asymmetric catalyst placement," in preparation for submission to $A C S$ journals.

NSD-Clark LB07012 New Experimental Initiative to Deduce $(\mathrm{n}, \mathrm{f})$ Cross Sections for Advanced Fuel Studies

M.S.Basunia, R.M.Clark et al; "Determining the $237 \mathrm{~Np}(\mathrm{n}, \mathrm{f})$ reaction cross section indirectly: Study of the absolute surrogate method in the 10 to $20 \mathrm{MeV}$ energy range," in preparation for submission to Phys. Rev. C.

S.R. Lesher, M.S. Basunia et al; "Benchmarking the surrogate ratio method with applications," to be published in Proceedings of International Conference on Nuclear Data for Science and Technology, April 2007, Nice, France

NSD-Lesko/Wang LB07020 Physics Detector and Sensor Technologies Applied to Geological and Geophysical Applications at DUSEL

J.S.Y. Wang; "Earth Science Collaborations for Deep Underground Science and Engineering Laboratory," Rock Mechanics: Meeting Society's Challenges and Demands 2, 1105-

1114, ed. E. Eberhardt, D. Stead, T. Morrison; Taylor \& Francis Group, London (2007).

NSD-Phair LB05030 Improved Spectroscopy of Weakly Bound States in Nuclei

M. Wiedeking, P. Fallon, A. O. Macchiavelli et al; "Lifetime measurement of the first excited $2^{+}$state in ${ }^{16} \mathrm{C}$," submitted to Phys. Rev. Lett.

M. Wiedeking, P. Fallon, A. O. Macchiavelli et al; "Nuclear Structure of ${ }^{18} \mathrm{~N}$ and the Neighboring $N=11$ Isotones," submitted to Phys. Rev. $C$. 
B. F. Lyles, L. A. Bernstein, J. T. Burke et al; "Absolute and relative surrogate measurements of the ${ }^{236} \mathrm{U}(n, f)$ cross section as a probe of angular momentum effects," Phys. Rev. C 76, 014606 (2007).

C. W. Beausang, S. R. Lesher, J. T. Burke et al; "New Results on Fission Cross Sections in Actinide Nuclei using the Surrogate Ratio Method and on Conversion Coefficients in Triaxial Strongly Deformed Bands in ${ }^{167}$ Lu from ICE Ball and Gammasphere," Acta Physica Polonica B 38, 1535 (2007).

R. Hatarik, L. A. Bernstein, J. T. Burke et al; "Is ( $d, p \quad$ ) a surrogate for neutron capture?" to be published in Proceedings of Compound-Nuclear Reactions and Related Topics, Yosemite (2007).

J. Gibelin, L. Phair, M. Wiedeking et al; "Fusion evaporation reactions as a tool for light nuclei gamma spectroscopy," to be published in Proceedings of Compound-Nuclear Reactions and Related Topics, Yosemite (2007).

NSD-Sichtermann LB07027 Hyperons in Polarized Proton Collisions and the Origin of the Nucleon Spin

Q.H. Xu, for the STAR Collaboration; "Longitudinal Spin Transfer in Inclusive Lambda and anti-Lambda Production in Polarized Proton-Proton Collisions at s**(1/2) $=200 \mathrm{GeV}$," AIP Conf. Proc. 915, 428-431 (2007, SPIN-2006 Conference), LBNL-62052.

Y. Chen, Z.T. Liang, E.P. Sichtermann, Q.H. Xu, S.S. Zhou; "Anti-Hyperon Polarization in High Energy pp Collisions with Polarized Beams," submitted for publication to Phys. Rev. D, LBNL-63097, hep-ph 0707.0534.

NSD-Vetter LB06025 Development of a Low-Energy, High-Current Astrophysics Accelerator

D.S. Todd, D. Leitner, M. Leitner, R. Maruyama, P.A. Vetter, K.N. Xu; "Design of the low energy astrophysics research facility CLAIRE," Nuclear Inst. and Methods in Physics Research B 261 (1-2), 544-8 (2007).

D. Leitner, P. Vetter; "Working group A5 Underground Accelerator Laboratory for Nuclear Astrophysics," White Paper for DUSEL Town Meeting, Washington, DC, November 2-4, 2007. Unpublished, http://ecrgroup.lbl.gov/white_paper.html

P.A. Vetter, D. Leitner, M. Leitner, D. S. Todd; "Need for a New Nuclear Astrophysics Accelerator," Nuclear Science Advisory Committee/American Physical Society Division of Nuclear Physics Town Meeting, Chicago, IL Jan. 19-21, 2007.

PBD-Adams LB07035 Research Tools for the Conversion of Cellulose to Ethanol: Structural Studies of Cellulose Synthesis

S Persson, A Paredez, A Carroll, H Palsdottir, M Doblin, P Poindexter, N Khitrov, M Auer, C Somerville; "Genetic evidence for three unique positions in primary cell wall cellulose synthase (CESA) complexes in Arabidopsis," PNAS 104 (39), 15566-71 (2007), PMID: 17878302.

$\underline{\text { PBD-Crooks LB05044 Statistical Dynamics of Protein Evolution }}$

G.E. Crooks, "Beyond Boltzmann-Gibbs Statistics: Maximum Entropy Hyperensembles OutOf-Equilibrium," Phys. Rev. E 75, 041119 (2007). 
G.E. Crooks, C. Jarzynski; "Work Distribution For The Adiabatic Compression Of A Dilute And Interacting Classical Gas," Phys. Rev. E 75, 021116 (2007).

G.E. Crooks, "Measuring Thermodynamic Length," Phys. Rev. Lett. 99, 100602 (2007).

PBD-Frei et al LB06006 Light Regulation and Visible Light-Driven Water Oxidation in Engineered Photosystems

W.W. Weare, Y. Pushkar, V.K. Yachandra, H. Frei; "Electron Transfer from Mn Dimer Complex to Cr Centers in Silica Nanopore Driven by Visible Light," submitted to J. Am. Chem. Soc.

D.A. Fletcher, "Unilamellar Vesicle Encapsulation by Microfluidic Jetting," in preparation for submission to J. Phys. Chem. B.

S. Pronk, P.L. Geissler; "A Computational Model for Actin Network Elasticity Comprising Discrete, Fluctuating Filaments with Nonlinear Mechanical Response," in preparation for submission to J. Phys. Chem. B.

PBD-Schaffer et al LB07024 Cooperation of Biochemical and Mechanical Signals in Regulating Cell Fate Decision During Tissue Morphogenesis

C. Pangarkar, D. Schaffer; "Systems Biology Approaches to Studying Stem Cell Fate," in preparation for submission to IET Systems Biology.

PD-Battaglia LB05002 Advanced Monolithic Silicon Pixel Detectors

M. Battaglia, "Response simulation of CMOS pixel sensors for the ILC vertex tracker," Nucl. Instrum. Meth. A 572, 274 (2007).

M. Battaglia, D. Contarato, P. Giubilato, L. Greiner, L. Glesener, B. Hooberman; “A study of monolithic CMOS pixel sensors back-thinning and their application for a pixel beam telescope," Nucl. Instrum. Meth. A 579, 675 (2007) [arXiv:physics/0611081].

M. Battaglia, D. Bisello, D. Contarato, P. Denes, P. Giubilato, L. Glesener, C. Vu; "A Monolithic Pixel Sensor in 0.15 micron Fully Depleted SOI Technology," Nucl. Instrum. Meth. A 583, 526 (2007) [arXiv:0709.4218 [physics.ins-det]].

M. Battaglia, D. Contarato, P. Giubilato, L. Glesener, L. Greiner, B. Hooberman; "Particle Tracking with a Thin Pixel Telescope," to appear in the Proceedings of the Intl. Linear Collider Conference, LCWS07, Hamburg, May 2007 and arXiv:0710.0033 [physics.insdet].

D. Contarato, M. Battaglia, J.-M. Bussat, P. Denes, P. Giubilato, L. Glesener, B. Hooberman, C.Q. Vu; "Monolithic Pixels R\&D at LBNL," to appear in the Proceedings of the Intl. Linear Collider Conference, LCWS07, Hamburg, May 2007 and arXiv:0710.0361 [physics.ins-det].

M. Battaglia, J.-M. Bussat, D. Contarato, P. Denes, P. Giubilato, L. Glesener; "Development of CMOS monolithic pixel sensors with in-pixel correlated double sampling and fast readout for the ILC," to appear in the Conference Record of the 2007 IEEE Nuclear Science Symposium, Honolulu HI, October 2007. 
PD-Bauer LB07004 Soft-collinear Effective Theories Applied to Collider Physics

Z. Ligeti, F. J. Tackmann, Precise predictions for B ---> X(s) 1+ 1- in the large $\mathrm{q}^{* *} 2$ region," Phys. Lett. B 653, 404-410 (2007).

R. Enberg, P.J. Fox, L.J. Hall, A.Y. Papaioannou, M. Papucci; "LHC and dark matter signals of improved naturalness," JHEP 0711, 014 (2007). LBNL-62748, UCB-PTH-07-10.

P.J. Fox, A. Rajaraman, Y. Shirman; "Bounds on Unparticles from the Higgs Sector," Phys. Rev. D 76, 075004 (2007), LBNL-62680, UCI-TR-2007-23.

P.J. Fox, Z. Ligeti, M. Papucci, G. Perez, M.D. Schwartz' "Deciphering top flavor violation at the LHC with B factories," UCB-PTH-07-06, YITP-SB-07-11.

K.S.M. Lee, Z. Ligeti, I.W. Stewart, F.J. Tackmann; "Extracting short distance information from b ---> s 1+ 1- effectively," Phys. Rev. D 75, 034016 (2007), CALTECH-MAP-330, LBNL-62077, MIT-CTP-3793.

N.J. Craig, P.J. Fox, J.G. Wacker; "Reheating Metastable O'Raifeartaigh Models," Phys. Rev. $D$ 75, 085006 (2007), SLAC-PUB-12215,

S. Chang, P.J. Fox, N. Weiner; "Visible Cascade Higgs Decays to Four Photons at Hadron Colliders," Phys. Rev. Lett. 98, 111802 (2007).

C.W. Bauer, F.J. Tackmann; "Gaining analytic control of parton showers," Phys. Rev. D 76, 114017 (2007).

C.W. Bauer, M.D. Schwartz; "Event Generation from Effective Field Theory," Phys. Rev. D 76, 074004 (2007).

PD-Murayama LB05027 New Directions for Theoretical Physics at the TeV-Scale

M. Aganagic, C. Beem, B. Freivogel; "Geometric Metastability, Quivers and Holography," Aug 2007, e-Print: arXiv:0708.0596 [hep-th].

B. Freivogel, G.T. Horowitz, S. Shenker; "Colliding with a crunching bubble," JHEP 0705, 090 (2007), SU-ITP-07-03, UCB-PTH-07-04. e-Print: hep-th/0703146.

R. Bousso, B. Freivogel; "Paradox in the global description of the multiverse," JHEP 0706, 018 (2007), e-Print: hep-th/0610132.

E.G. Gimon, T.S. Levi; "Black Ring Deconstruction,” Jun 2007, e-Print: arXiv:0706.3394 [hep-th].

E.G. Gimon, P. Horava; "Astrophysical violations of the Kerr bound as a possible signature of string theory," Jun 2007, e-Print: arXiv:0706.2873 [hep-th]

E.G. Gimon, T.S. Levi, S.F. Ross; "Geometry of non-supersymmetric three-charge bound states,” JHEP 0708, 055 (2007), DCPT-07-19, May 2007, e-Print: arXiv:0705.1238 [hepth].

V. Balasubramanian, J. de Boer, V. Jejjala, J. Simon; "Entropy of near-extremal black holes in AdS(5)," UPR-T-1185, ITFA-2007-33, DCPT-07-37, UCB-PTH-07-13, Jul 2007, ePrint: arXiv:0707.3601 [hep-th].

V. Balasubramanian, B. Czech, K. Larjo, D. Marolf, J. Simon; “Quantum geometry and gravitational entropy,” JHEP 0712, 067 (2007), UPR-T-1182, UCB-PTH-07-09, May2007, e-Print:arXiv:0705.4431 [hep-th].

V. Balasubramanian, B. Czech, V.E. Hubeny, Klaus Larjo, M. Rangamani , J. Simon; "Typicality versus thermality: An Analytic distinction," UPR-T-1170, DCPT-07-01, UCB-PTH-07-01, LBNL-62258, NSF-KITP-07-02, Jan 2007; e-Print:hep-th/0701122. 
Cross-Div.-Lester et al LB05020 Development and Application of Quantum Monte Carlo (QMC) Methods to Biological Systems

H. M. Cho, W. A. Lester, Jr., G. Fleming, M. Frenklach; “An Explicit Solvent Model for Quantum Monte Carlo: Combined Quantum Monte Carlo and Molecular Mechanics Approach," in preparation for submission to J. Chem. Phys.

R. Salomon-Ferrer, W. A. Lester, Jr., G. Fleming, M. Frenklach; "Quantum Monte Carlo Study of Ethylene-Ethylene Triplet-Triplet Energy Transfer," in preparation for submission to J. Chem. Phys.

H. M. Cho, W. A. Lester, Jr., G. Fleming, M. Frenklach; "Combined Quantum Monte Carlo and Molecular Mechanics (QMC/MM) Study of the Internal Rotational Barrier of Formamide in Water, " in preparation for submission to J. Chem. Phys.

R. Salomon-Ferrer, W. A. Lester, Jr., G. Fleming, M. Frenklach; "Constrained DFT TripletTriplet Energy Transfer," in preparation for submission to J. Chem. Phys. 